\title{
High-Resolution Fluorescence Microscopy with Photoswitchable Fluorescent Proteins
}

\author{
Dissertation \\ zur Erlangung des mathematisch-naturwissenschaftlichen Doktorgrades \\ "Doctor rerum naturalium" \\ der Georg-August-Universität Göttingen
}

vorgelegt von

Hannes Bock

aus Hamburg

Göttingen, 2008 
D 7

Referent: Prof. Dr. M. Münzenberg

Koreferent: Prof. Dr. S. W. Hell

Tag der mündlichen Prüfung: 


\section{High-Resolution Fluorescence Microscopy with Photoswitchable Fluorescent Proteins}




\section{Summary}

The diffraction limit in far-field fluorescence microscopy can be overcome by photoswitching the marker molecules between a fluorescent and a non-fluorescent state. Photoswitching enables the successive generation and readout of nano-sized fluorescent areas in the sample. Subsequently, these areas can be assembled to form a super-resolution image of the specimen. For the first time in this work, one and the same molecular switching mechanism of a reversibly switchable fluorescent protein (RSFP) was used to realize two alternative variations of this general nanoscopy principle: one based on the targeted switching of ensembles of molecules and the other based on the stochastic switching of single emitters. RSFPs are of particular interest for these techniques because they allow the non-invasive study of processes in living cells and can be efficiently switched using low light intensities, thus minimizing photo stress and possible photo-induced damage of the cell. Furthermore, the protein's photoswitching properties can be improved via targeted mutagenesis. In the case of both nanoscopy techniques presented here, image acquisition could be significantly improved and accelerated as compared to related approaches. For the first time, two-color far-field fluorescence nanoscopy based on the photoswitching of single emitters was successfully realized in biological samples.

\section{Zusammenfassung}

Die Beugungsgrenze in der Fluoreszenzfernfeldmikroskopie kann durch lichtinduziertes Schalten der verwendeten Farbstoffmoleküle zwischen einem fluoreszenten und einem nichtfluoreszenten Zustand überwunden werden. Dazu werden in der Probe durch Fotoschalten nacheinander fluoreszente Bereiche erzeugt und ausgelesen, deren Ausdehnung nicht durch die Wellenlänge des Lichtes begrenzt ist. Durch das nachträgliche Zusammensetzen dieser nanoskopischen Bereiche entsteht ein hochaufgelöstes Bild der Probe. In dieser Arbeit konnten zum ersten Mal mit ein und demselben reversibel schaltbaren Fluoreszentprotein (RSFP) zwei verschiedene Umsetzungen dieses allgemeinen Prinzips realisiert werden, die zum einen auf dem gezielten Schalten von Ensemblen von Molekülen und zum anderen auf dem zufälligen Schalten einzelner Moleküle beruhen. RSFPs sind für diese Verfahren von großer Bedeutung: Sie können Prozesse in lebenden Zellen nichtinvasiv sichtbar machen; sie können bei niedrigen Lichtintensitäten effektiv geschaltet werden, was die Einwirkung auf die Zelle minimiert; und sie können in ihren Eigenschaften als Fotoschalter durch Mutagenese gezielt verbessert werden. Beide der hier vorgestellten Nanoskopieverfahren stellen in ihrer Umsetzung durch eine erhebliche Verminderung der Bildaufnahmezeit eine deutliche Verbesserung im Vergleich zu früheren Ansätzen dar. Zusätzlich wurde zum ersten Mal einzelmolekülbasierte Zwei-Farben-Nanoskopie in biologischen Proben realisiert. 


\section{Contents}

\begin{tabular}{ll}
\hline Introduction & 6
\end{tabular}

1 Resolution in Optical Microscopy 9

1.1 The Diffraction Limit . . . . . . . . . . . . . . . . . . . . . . . . . . 9

1.2 Sub-Diffraction Techniques . . . . . . . . . . . . . . . . . . 11

$1.2 .1 \quad$ RESOLFT Microscopy $\ldots \ldots \ldots \ldots \ldots \ldots$

$1.2 .2 \quad$ Single Molecule Switching Microscopy $\ldots \ldots \ldots \ldots \ldots$

$\begin{array}{lll}2 & \text { The RSFP rsFastLime } & 18\end{array}$

2.1 Reversible Switching at the Ensemble- and Single Molecule Level . . . . . 20

2.2 RESOLFT Microscopy with rsFastLime $\ldots \ldots \ldots \ldots \ldots \ldots$

$2.2 .1 \quad$ Imaging with rsFastLime $\ldots \ldots \ldots \ldots \ldots \ldots$

2.3 Detailed Ensemble Photophysics of rsFastLime . . . . . . . . . . . . . 47

$2.4 \quad$ Single Molecule Switching (SMS) Microscopy with rsFastLime $\ldots . . . \quad 54$

2.5 Summary of RESOLFT- and SMS Microscopy with rsFastLime $\ldots \ldots 63$

3 Two-Color Single Molecule Switching Microscopy 68

3.1 Dark State Shelving of Cy5 $\ldots \ldots \ldots \ldots \ldots \ldots$

3.2 Two-color Imaging of Biological Samples . . . . . . . . . . . . . 71

4 The RSFP ASFP595

4.1 Photoswitching Properties $\ldots \ldots \ldots \ldots \ldots \ldots \ldots$

4.2 Super-Resolution RESOLFT Imaging $\ldots \ldots \ldots \ldots \ldots \ldots \ldots$

5 Conclusion $\quad 80$

\begin{tabular}{ll}
\hline Bibliography & 82
\end{tabular}

\begin{tabular}{ll}
\hline A Appendix & 92
\end{tabular}

A.1 Expressions for the Resolving Power . . . . . . . . . . . . . . . . 92

A.2 Photophysical Model of rsFastLime . . . . . . . . . . . . . . 93

A.3 Staining of Tubulin in PtK2 Cells . . . . . . . . . . . . . . . . . . . 94

A.4 List of Scientific Contributions . . . . . . . . . . . . . . . . . . . . . 95

\begin{tabular}{ll}
\hline B Danksagung & 96
\end{tabular} 



\section{Introduction}

By pushing open the door to a previously unknown world, the invention of the far-field light microscope in the 16. and 17 . century truly marked a milestone in the history of science. In the centuries to follow it lead scientists to major new insights ranging from the discovery of the cell as the basic structural and functional unit of living tissue to the observation of statistical molecular motion by R. Brown (now known as Brownian motion), to name only a few. Since then the far-field light microscope has found a wide scope of applications, particularly in the biomedical sciences.

Following the seminal work of Ernst Abbe in the second half of the nineteenth century [1] it became commonly accepted that the resolving power of the light microscope was limited by diffraction to about half the optical wavelength, i.e. typically to $\approx 200 \mathrm{~nm}$ in the lateral and $\approx 500 \mathrm{~nm}$ in the axial direction. Subsequently, the twentieth century brought about the development of imaging technologies capable of resolving structures on the atomic scale. E.g., the transmission electron microscope (TEM) [2] makes use of the short de Broglie wavelength of accelerated electrons $(\approx 100 \mathrm{keV})$. In scanning tunneling microscopy (STM)[3] a conducting tip measures the tunneling current as the tip is moved over the surface of the (conducting) sample. In an atomic force microscope (AFM) [4], on the other hand, it is the interaction between the scanning tip and the surface atoms that provides the information about the surface structure.

All of these methods have spurred scientific breakthroughs. However, although they provide a more than $\approx 1000$-fold improved resolution over optical microscopy, they suffer from a number of limitations when it comes to the imaging of biological specimens. Namely, they are mostly restricted to the study of the sample surface or necessitate cutting it into thin slices; also, they generally require a sample preparation protocol which is not compatible with the imaging of living objects, including e.g. de-hydration, evacuation or cooling of the specimen to cryogenic temperatures.

Not being subjected to any of the aforementioned restraints, far-field fluorescence microscopy has emerged as one of the most powerful and versatile tools in the life sciences in the course of the past decades. The methodology relies on the specific staining of target proteins or organelles with fluorescent labels in combination with excitation of the fluorescent marker by a light source of a suitable wavelength, typically operating in the visible spectrum between 450 and 700nm. Thus, it bears the significant advantage of permitting true three dimensional imaging of translucent specimens. Even more importantly, far-field fluorescence microscopy enables the imaging of living cells. What adds to its great capacity is a highly developed labeling technology that allows to mark defined proteins or cellular compartments with high efficiency and specificity.

In this work, the final remaining drawback of far-field fluorescence microscopy is addressed, namely its limited resolving power which until recently prevented the study of nanoscale 
structures and processes occurring in living cells. However, in the last decade a number of nanoscopy methodologies based on the photoswitching of the fluorescent labels have emerged which are capable of breaking the diffraction limit of far-field light microscopy formulated by Abbe about a century and a half ago [5]. It can be anticipated that in the near future further progress in imaging technology in connection with the development and refinement of new switchable fluorescent probes will significantly enhance our knowledge about fundamental cellular processes. 


\section{Resolution in Optical Microscopy}

\subsection{The Diffraction Limit}

As a noninvasive imaging technique which allows the observation of living specimens farfield fluorescence microscopy has gained great importance in recent decades. However, it suffers from its limited resolution. The resolution of an imaging apparatus is defined as the minimum distance at which two similar objects can still be discerned. Consequently, the device is not capable of revealing the details of structures which are smaller than this minimum resolvable distance. E. Abbe was the first to point out that in a far-field light microscope diffraction fundamentally limits the resolution ability to a scale which is set by the optical wavelength [1]. Specifically, he stated that the lateral resolution $\Delta r$ and the axial resolution $\Delta z$ of such an instrument can be expressed as

$$
\Delta r \approx \frac{\lambda}{2 n \sin (\alpha)} \quad \text { and } \quad \Delta z \approx \frac{2 \lambda}{n \sin ^{2}(\alpha)}
$$

where $\lambda$ is the wavelength of the light used in the image formation process, $n$ is the index of refraction of the surrounding medium and $\alpha$ the semi aperture angle of the objective lens. The quantity $N A=n \sin (\alpha)$ is commonly referred to as the numerical aperture of the system. Nowadays, high aperture oil-immersion objective lenses feature numerical apertures of $N A \approx 1.4$. According to Abbe, (1.1) denotes both the extension of the far-field optical image of a point-like source and the width of the smallest possible focus to which a beam of light can be brought using the respective instrument. The spatial intensity distribution $h(\mathbf{r})$ of the image of a point-like object completely characterizes an optical system and is denoted the point spread function (PSF) of the device. In a far-field light microscope the image $J(\mathbf{r})$ is given by the convolution of the object $O(\mathbf{r})$ with the PSF $h(\mathbf{r})$, i.e.

$$
J(\mathbf{r})=O(\mathbf{r}) \otimes h(\mathbf{r}) .
$$

Following convolution theory, object and image are identical only if the PSF is given by a Dirac delta function. As long as it features a finite width the convolution operation always leads to an effective blurring of the object, i.e. to a loss of information about its structure. Thus, the PSF's full width at half maximum (FWHM) serves as a measure of the resolving power of an optical instrument. In order to illustrate the relation between PSF size and image resolution, figure 1.1 depicts a simulation of the image formation process carried out with two PSFs of different size, namely $\Delta r=50 \mathrm{~nm}(\mathbf{A})$ and $\Delta r=200 \mathrm{~nm}(\mathbf{B})$, where $\Delta r$ denotes the FWHM of the PSF. The sample is given by a 2D random distribution of bead-like objects with a diameter of $40 \mathrm{~nm}$. $200 \mathrm{~nm}$ is approximately equal to the resolving power which can be achieved with visible light in a diffraction-limited far-field microscope. On the other hand, a 

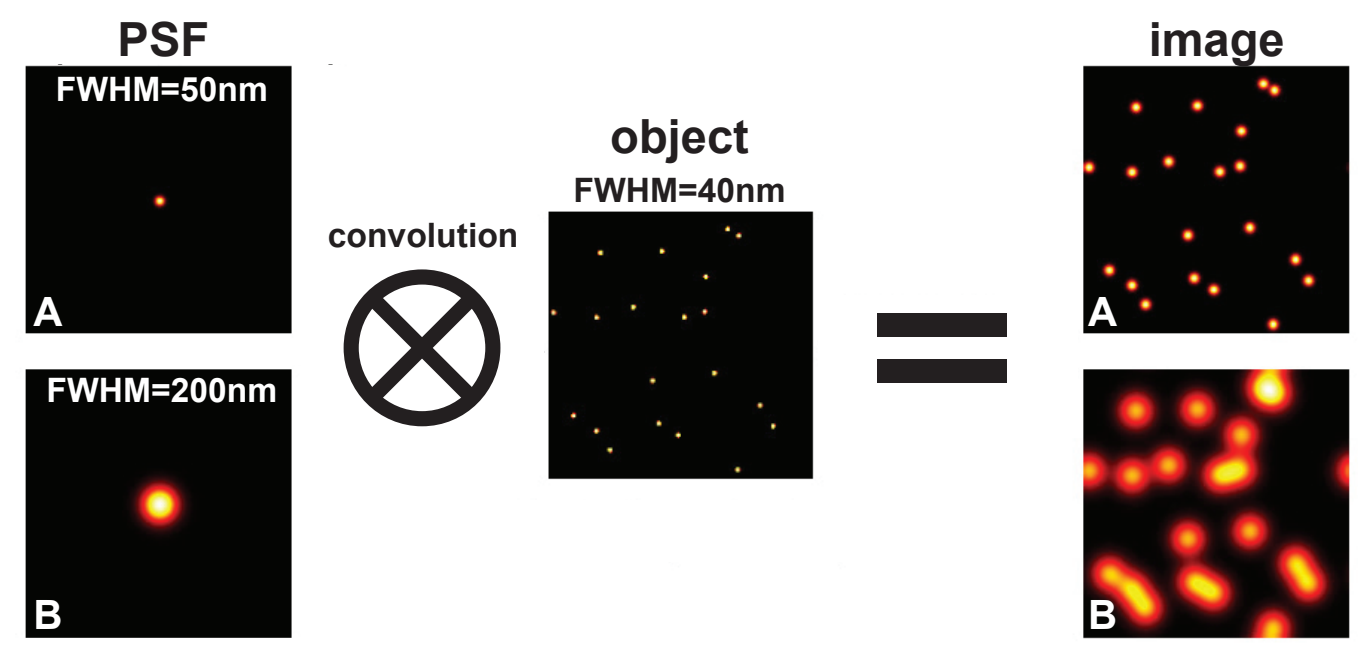

Figure 1.1: Imaging of an object with PSFs of different lateral extent $\Delta r(\mathbf{A}+\mathbf{B})$. Convolution of the object with a PSF of finite size yields a blurred image. Decreasing the PSF width $\Delta r$ enhances the resolving power of the microscope.

PSF width of 50nm represents the potential of an imaging device operating at subdiffraction resolution. Clearly, the 50nm PSF shown in A resolves all objects whereas the 200nm PSF used in $\mathbf{B}$ fails to properly image the closest particles.

In the course of the past decades, a number of attempts have been made to increase the resolution in far-field imaging. An important step forward in this context was the invention of the confocal microscope [6], which greatly enhanced the 3D imaging capability of light microscopy. A confocal microscope is a laser scanning system with a pinhole inserted in the detection plane. This way, it features essentially symmetric excitation and detection paths. As a result, the effective point spread function of a confocal system is given by the product of the excitation- and the detection PSF, $h_{\text {conf }}=h_{e x c} \cdot h_{\text {det }}$, which leads to an efficient suppression of out-of-focus light. In connection with fast beam scanning, confocal microscopy has developed into a widely used tool in fluorescence imaging as well as in single molecule spectroscopy [7].

A technique which further enhances the axial resolution in far-field fluorescence imaging is 4Pi-microscopy [8]. In a 4Pi microscope two opposing lenses which are employed for coherent illumination and/or detection effectively increase the numerical aperture of the system and achieve an axial resolution of down to $\Delta z \approx 80 \mathrm{~nm}[9]$ constituting an improvement of a factor of $\approx 7$ over confocal microscopy. A related approach, $\mathrm{I}^{5} \mathrm{M}$ microscopy [10], was shown to reach a resolution of $\approx 100 \mathrm{~nm}$ along the optical axis.

Multi-photon microscopy [11] is another innovative technique which has been widely accepted as the method of choice for deep tissue imaging. A multi-photon microscope relies on excitation photons carrying only a fraction $(1 / 2,1 / 3, \ldots)$ of the energy needed to excite the fluorophore. Consequently, an excitation process can occur only upon the simultaneous 
absorption of several photons. To achieve the high photon density necessary to induce multiphoton absorption, intensities in the range of $\approx 100 \mathrm{GW} / \mathrm{cm}^{2}$ are typically required. Due to the high non-linearity of the process, excitation effectively only takes place in the very center of the focus, making the use of a confocal pinhole unnecessary. The reduced size of the excitation spot is usually outweighed by the longer wavelength; as a result the resolution of a multi-photon microscope is comparable to that of a confocal system.

A straightforward way of improving the resolving power of a light microscope is to resort to shorter wavelengths (see equation (1.1)). However, this approach is often not compatible with life cell imaging as ultra violet light may be phototoxic to live cells. Other problems include an elevated level of autofluorescence, caused e.g. by fluorescing amino acids. Additional complications arise because standard optical elements cannot be used at wavelengths below $\approx 350 \mathrm{~nm}$.

Finally, optical microscopy was extended to near field imaging as implemented in scanning near-field optical microscopy (NSOM). In this method, light is transmitted through a subdiffraction sized aperture or tip which is scanned over the object at a distance of only a few nanometers, enabling imaging at resolutions down to $\approx 10 \mathrm{~nm}[12]$. Yet, the technology remains restricted to the measurement of surfaces significantly limititing its role as an imaging methodology in the life sciences.

\subsection{Sub-Diffraction Techniques}

The techniques presented in the preceding section pushed diffraction in fluorescence microscopy to its limits. In all of them, however, the wavelength continues to set the scale for the attainable resolution. Breaking this limit required a fundamentally new insight [13]: the story of how an image is formed really does not end with the optics involved in the imaging process. Rather, the entities to be imaged and their response to the applied illumination form a vital constituent of the microscope. More specifically, all fluorescence nanoscopy techniques which have been realized to date rely on the existence of a signal-generating 'bright' state $A$ and a 'dark' state $B$ of the fluorescent marker and the possibility to use light to 'switch' the marker between these two states. This property can then be used to sequentially single out and record 'bright' features of sub-diffraction size. Knowing their coordinates $r_{i}$ makes it possible to assemble these features to form an image with sub- $\lambda$ resolution.

Until today two different strategies aimed at implementing this common fundamental concept have been pursued, namely reversible saturable optically linear (fluorescence) transition (RESOLFT)- microscopy and single molecule switching (SMS)- microscopy. Both differ in

- the way the coordinate $\mathbf{r}_{i}$ of the sub-diffraction sized feature singled out within one time sequence is determined (directed scanning in RESOLFT microscopy versus stochastic scanning followed by localization in SMSM) and

- the modality used within one time sequence to generate the sub-diffraction sized volume at $\mathbf{r}_{i}$ (switching molecular ensembles (RESOLFT) versus switching single molecules $(\mathrm{SMSM}))$. 
In the following the basic principles of RESOLFT- and SLM- microscopy shall be introduced and a brief summary of the accomplishments as well as the current state of the two techniques be given.

\subsubsection{RESOLFT Microscopy}

The first family of concepts capable of breaking the diffraction limit in far-field fluorescence imaging was RESOLFT-microscopy, the acronym RESOLFT standing for reversible saturable optically linear (fluorescence) transition [14, 15]. It is a scanning method in which the specimen is sampled with an effectively sub-diffraction sized focal spot. As is implied by the acronym, the RESOLFT principle is based upon the possibility to reversibly transfer the marker molecule between a signal-generating 'bright' state $A$ and a 'dark' state $B$. Additionally, it is essential that one of the transitions, $A \rightarrow B$ say, be light driven and saturable. Here, 'saturable' means that for sufficiently high intensities $I$ ( $I$ being the intensity driving the transition $A \rightarrow B$ ) practically all marker molecules are transferred from $A$ to $B$. This implies that the relative population $n_{A}$ of marker molecules remaining in state $A$ is a non-linear function of the applied intensity $I$.

\section{Underlying Mechanism}

The simplest conceivable realization of such a system is depicted in figure 1.2 a. Marker molecules are driven from the 'bright' state $A$ to the 'dark' state $B$ by the intensity-dependent rate $k_{A B}=\sigma_{A B} \gamma I$ measured in $1 / \mathrm{s}$, where $\sigma_{A B}$ is an absorption cross section measured in $\mathrm{cm}^{2}$, $\gamma=\lambda / h c$ is the inverse photon energy of the driving light measured in $1 / J$ ( $\lambda$ : wavelength, $h$ : Planck's constant, $c$ : vacuum speed of light) and $I$ is the intensity of the driving light measured in $W / \mathrm{cm}^{2}$. The reverse rate $k_{B A}$ driving marker molecules from state $B$ back to state $A$ is considered constant (due to e.g. thermal recovery). The dynamics of this two state model is described by the rate equation

$$
\dot{n}_{A}=-\dot{n}_{B}=-k_{A B} n_{A}+k_{B A} n_{B}=-\sigma_{A B} \gamma I n_{A}+k_{B A} n_{B}
$$

where $n_{A}$ and $n_{B}$ denote the relative state populations. With the additional boundary conditions $n_{A}+n_{B}=1$ (particle conservation) and $n_{A}(t=0)=1$ (all markers initially in state $A$ ) the unique solution of 1.3 is given by

$$
n_{A}(t)=\frac{k_{B A}}{k_{A B}+k_{B A}}+\frac{k_{A B}}{k_{A B}+k_{B A}} \exp \left(-\left(k_{A B}+k_{B A}\right) t\right) .
$$

Assuming continuous wave illumination (i.e. $I(t)=$ const), after a time period $\Delta t>>$ $\left(k_{A B}+k_{B A}\right)^{-1}$ the 'bright' state population $n_{A}$ reaches its equilibrium value (in all of the following $n_{A}$ may denote the equilibrium 'bright'-state population)

$$
n_{A}=\frac{k_{B A}}{k_{A B}+k_{B A}}=\frac{k_{B A}}{\sigma_{A B} \gamma I+k_{B A}}=\frac{1}{1+I / I_{s a t}} .
$$


At the saturation intensity $I_{\text {sat }}$, defined as

$$
I_{s a t}:=k_{B A} /\left(\sigma_{A B} \gamma\right),
$$

50 percent of the marker molecules have been transferred from $A$ to $B$. Thus the saturation intensity $I_{\text {sat }}$ is a measure of the driving intensity $I$ needed to outperform the competing reverse transition rate $k_{B A}$. A graph displaying the saturable non-linear dependence (1.5) of the 'bright' state equilibrium population $n_{A}$ on the driving intensity $I$ is shown in figure $1.2 \mathrm{~b}$ (note the logarithmic scale on the abscissa). For $I \gg I_{\text {sat }}$ practically all marker molecules are transferred to the 'dark' state $B$.

\section{Implementation for Sub-diffraction Imaging}

The way in which the existence of a reversible saturable transition in the marker molecule can be exploited to shape a signal-generating volume of sub-diffraction size at a pre-defined location $x_{0}$ is illustrated in figure 1.2c. The light grey area (1) represents the conventional, diffraction-limited point spread function (PSF) $h(x)$ of a signal-generating (excitation-) beam focused at $x_{0}$. The idea underlying RESOLFT-type imaging is to reduce the signal-volume by inducing and saturating the transition $A \rightarrow B$ from the 'bright' to the 'dark' marker state everywhere but at $x_{0}$. The latter can be achieved by applying a spatially modulated intensity profile $I(x)$ featuring zero intensity at $x_{0}$ (i.e. $I\left(x_{0}\right)=0$ ). In figure $1.2 \mathbf{b} I(x)$ is represented by the black curves numbered 2), 3) and 4), each corresponding to a different value of the maximum intensity $I_{\max }$. By driving the transition $A \rightarrow B$ further and further into saturation the signal-generating volume centered at $x_{0}$ can be made arbitrarily small: increasing $I_{\max }$ results in an ever steeper rise (i.e. spatial gradient) of $I$ near $x_{0}$ (black curves 2), 3) and 4) in $1.2 \mathrm{c}$ ); consequently, a molecule at $x_{0}+\delta$, where $\delta$ may be any arbitrarily small distance, is efficiently transferred to the 'dark' state $B$ if only $I\left(x_{0}+\delta\right)>>I_{\text {sat }}$.

The volume-reduction of the effective PSF with rising peak intensity $I_{\max }$ is represented by the the grey shaded areas marked (2), (3) and (4) in figure $1.2 \mathrm{c}$, respectively. Each of the effective PSFs on display is the (point wise) product of the 'bright'-state population $n_{A}(x)=n_{A}(I(x))$ and the diffraction-limited (excitation-) PSF $h(x)$ :

$$
h^{\prime}(x)=n_{A}(I(x)) \cdot h(x)
$$

A measure of the resolution in a RESOLFT-type microscope is the full width at half maximum (FWHM) of the effective PSF (1.7), which may be denoted $\Delta x^{\prime}$ (i.e. $h^{\prime}\left(\Delta x^{\prime} / 2\right)=1 / 2$ ). In the following, the approximate dependence of $\Delta x^{\prime}$ on the intensity of the applied inhibition light shall be derived in a back-of-an-envelope type of way. When neglecting higher order terms in the Taylor expansion of the even functions $I(x)$ and $h(x)$ at $x_{0}$, they may be written as:

$$
I(x)=I_{0} \cdot\left(\frac{x}{\Delta x / 2}\right)^{2} \quad \text { and } \quad h(x)=1-\frac{1}{2}\left(\frac{x}{\Delta x / 2}\right)^{2}
$$

where $\Delta x$ denotes the FWHM of $h(x)$, i.e. $h(\Delta x / 2)=1 / 2$. Determining the dependence $\Delta x^{\prime}(I)$ simply amounts to solving

$$
h^{\prime}\left(\Delta x^{\prime} / 2\right)=n_{A}\left(I\left(\Delta x^{\prime} / 2\right)\right) \cdot h\left(\Delta x^{\prime} / 2\right)=\frac{1}{2}
$$



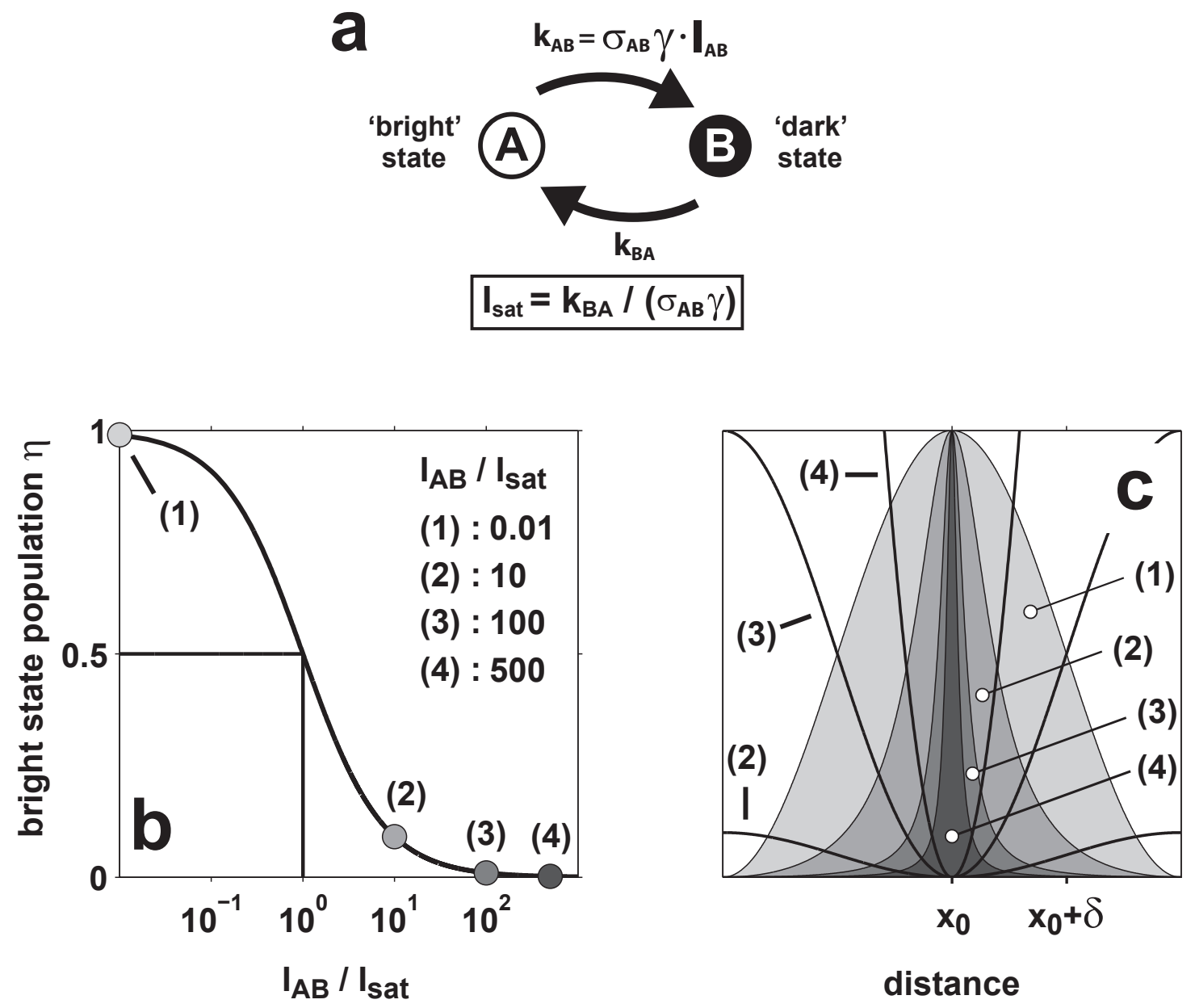

Figure 1.2: a Two-state model of a 'bright' state A and a 'dark' state $\mathrm{B}$. The transition $A \rightarrow B$ is driven by the intensity $I_{A B}$. b Characteristic inhibition curve $\eta\left(I_{A B}\right)$ of the transition $A \rightarrow B . \eta$ represents the population of the bright state A as a function of the 'switch-off' intensity $I_{A B}$. At the saturation intensity $I_{A B}=I_{s a t}$ state $A$ is depopulated by $50 \%$. b light grey (1): diffraction-limited PSF in a conventional microscope. (2)-(4): In a RESOLFT-type microscope the extent of the effective PSF can be made arbitrarily small by saturating the transition $A \rightarrow B$ with an intensity profile $I(x)$ featuring a zero at $x_{0}$ (black curves (2), (3) and (4)). The peak intensity values $I_{\max }$ of the black curves in $\mathbf{c}$ are those highlighted in $\mathbf{b}$.

for $\Delta x^{\prime}$. Using $n_{A}(I(x))$ from (1.5) in conjunction with (1.8) readily yields:

$$
\Delta x^{\prime}=\frac{\Delta x}{\sqrt{1+I_{0} / I_{s a t}}} .
$$

Clearly, 1.10 demonstrates $\Delta x^{\prime}$ can be made infinitely small by increasing the intensity $I_{0}$ of the inhibition light. It should be noted, though, that in a RESOLFT-type of microscope diffraction is in no way eliminated per se; both the signal-generating beam as well as the inhibition light pattern are still subject to Abbe's limit. Instead, the diffraction barrier is broken by saturating the transition $A \rightarrow B$ using a light pattern featuring an intensity zero. 


\section{Generality of the RESOLFT Concept and Current State of the Technique}

It is most important to point out the generality of the RESOLFT concept. All that is needed for its implementation is that the two marker states $A$ and $B$ yield distinguishable signals when being probed. E.g. it has been proposed that if one of them is absorbing, efficiently converting the absorbed photons into heat, the concomitant local change in refractive index could be probed by a laser beam providing a source for a non-fluorescence based detection mode [5]. Also, the principle is not restricted to sub-diffraction imaging but can equally be applied to the writing of nanoscale structures [15, 16] as has successfully been demonstrated [17, 18].

The first and to date most successful realization of the RESOLFT concept is the STED microscope, STED being an acronym for stimulated emission depletion [13, 19]. A subdiffraction fluorescence volume is shaped by first exciting marker molecules to the first excited singlet sate $S_{1}$; before fluorescence is emitted a second red-shifted beam with an intensity zero at the focal center de-excites marker molecules at the focal periphery via stimulated emission. Saturating the STED transition creates a sub-diffraction sized fluorescence volume. Up to now STED has achieved macromolecular scale resolution of $15-20 \mathrm{~nm}$ in the lateral direction [20] and has created three-dimensional focal spots of 40-45nm in diameter [21], both in biological samples. In addition, it has proved to be compatible with single molecule imaging [22]. Furthermore, STED microscopy has revealed synaptic vesicle movement in living cells at a lateral resolution of $62 \mathrm{~nm}$ imaged at video rate (28 frames per second) [23] and helped answer questions in biology which had previously not been accessible using standard fluorescence methods [24, 25]. Finally, STED microscopy is not restricted to high resolution imaging but has successfully been extended to fluorescence fluctuation spectroscopy with sub-diffraction volumes [26], revealing hitherto hidden details of lipid diffusion in the plasma membrane of living cells.

The power of STED microscopy is based on the ubiquity of the $S_{0} \rightarrow S_{1}$-transition in organic fluorophores, making the technique applicable to virtually all fluorescent dyes. In practice, however, it is somewhat restricted by the high laser powers needed to saturate the STED process $\left(\approx 100-1000 \mathrm{MW} / \mathrm{cm}^{2}\right)$. At these intensities photobleaching from higher excited molecular (triplet) states may result in a loss of the ability of the dye to fluoresce. These high intensities are required because in STED the process competing with stimulated emission is spontaneous fluorescence with typical rate constants of $k_{f l} \approx 10^{9} 1 / \mathrm{s}$. With the cross section for stimulated emission lying in the range of $\sigma_{S T E D} \approx 10^{-16} \mathrm{~cm}^{2}$, typical saturation intensities are $I_{\text {sat }}^{S T E D}=k_{f l} /\left(\gamma \sigma_{S T E D}\right) \approx 1-10 \mathrm{MW} / \mathrm{cm}^{2}$.

A realization of the RESOLFT-principle requiring smaller laser powers is ground state depletion (GSD) microscopy [27]. In GSD the fluorescence spot is reduced by shelving the marker molecules in the non-fluorescent triplet state common to all fluorescent dyes. The saturation intensity in this case is determined by how efficiently the triplet state $T_{1}$ can be populated via the first excited singlet state $S_{1}$ and by the triplet state live time. Typical saturation intensities are $I_{\text {sat }}^{G S D} \approx 100-1000 \mathrm{~W} / \mathrm{cm}^{2}$. Compared to STED, GSD has the further advantage that the same continuous wave $(\mathrm{CW})$ laser can be used for both triplet state shelving and 
fluorescence signal generation. Disadvantages are the high probability for photobleaching via the triplet state and a limited fluorescence signal. The latter is a consequence of the fact that the (desired) high efficiency of the shelving mechanism $\left(S_{1} \rightarrow T_{1}\right)$ entails an unwanted signal decay during fluorescence read-out. So far, GSD microscopy has successfully imaged the microtubular network in a mammalian cell and the distribution of proteins on the plasma membrane of a neuron at a lateral resolution of 50nm [28].

\subsubsection{Single Molecule Switching Microscopy}

In RESOLFT microscopy an individual marker molecule is bound to undergo several switching cycles in the course of the image acquisition process. This is a direct consequence of the fact that scanning is performed in a targeted manner and diffraction limited beams are employed to generate fluorescence volumes of subdiffraction size: to ensure that molecules at a predefined position are in state A neighboring markers have to be switched to state B [5]. Also, imaging at a higher resolution requires smaller scanning steps and hence more switching cycles per molecule. This may pose difficulties if the number of cycles per emitter is limited due to e.g. photo bleaching.

These problems are circumvented in a second class of super-resolution microscopy techniques which shall be summarized under the name Single Molecule Switching Microscopy (SMSM). They share the common idea of using molecular photoswitching to transfer only a small number of single markers at random positions in the sample to the bright state at a time. If the set of concomitantly activated molecules is so sparse that their diffraction limited images on a pixelated detector such as a CCD camera do not overlap their positions can be determined with subdiffraction accuracy. After fluorescence readout and localization the set of activated emitters is either irreversibly bleached or switched to an intermediate dark state. This process is repeated until the number of accumulated molecule positions allows an estimate of the marker distribution. Note that in order for an individual emitter to be localized it needs to undergo only a single switching cycle (off-on-off).

As early as 1930 Heisenberg was the first to point out that by calculating the center of mass of the diffraction limited image of a single emitter its position can be determined with an accuracy $\Delta r^{\prime}$ that is given by

$$
\Delta r^{\prime}=\frac{\Delta r}{\sqrt{N}}
$$

where $\Delta r$ is the full width at half maximum (FWHM) of the diffraction limited point spread function of the detection optics and $N$ is the number of detected photons [29]. In other words, the resolution in SMS microscopy varies with the brightness of the switchable fluorescent markers: brighter emitters readily yield an increased localization accuracy and hence a higher resolving power. Also, the anticipated resolution of an image can be tuned by displaying only those molecular positions which correspond to single molecule images containing a certain minimum number of photons. However, such a selection comes at the expense of a smaller total number of displayed single molecule positions and thus less complete information about the object to be imaged. In general a tradeoff between higher resolution on 
the one hand and more complete object information on the other must be made.

Clearly, SMS microscopy had its precursors. Indeed, single molecule localization to nearly $1 \mathrm{~nm}$ precision had already been demonstrated [30,31] and applied to the studies of molecular motor dynamics [32] before the advent of super-resolution single molecule microscopy techniques. Likewise, numerable studies on single particle localization- and tracking algorithms [33] as well as a detailed investigation of the influence of background noise and detector pixelation on the attainable localization accuracy [34] had been carried out. Yet, none of these works hinted at a mechanism which could potentially be used to sequentially read out fluorescence from an arbitrarily large number of molecules as is required for the recording of super-resolution images.

SMS microscopy was first introduced by Betzig and Hess [35] who sequentially activated, localized and irreversibly bleached sparse subsets of photoactivatable- or reversibly switchable fluorescent proteins [36, 37, 38, 39, 40] to image cellular proteins and organelles at a lateral resolution of $\approx 30 \mathrm{~nm}$. Their method, termed photoactivated localization microscopy (PALM), initially remained restricted to the imaging of thin sections and made use of total internal reflection fluorescence (TIRF) microscopy [41] to suppress diffuse background. Image acquisition times ranged from 2-10 hours, putting high demands on setup- and sample stability. Soon after other groups put forward related imaging methods calling them fluorescence photoactivation localization microscopy (FPALM) [42] and stochastic optical reconstruction microscopy (STORM) [43]. Similar to PALM, FPALM has relied on photoactivatable fluorescent proteins whereas STORM uses donor-acceptor pairs of switchable organic dyes [44, 45, 46]. All of the aforementioned techniques employ separate lasers for synchronized activation and fluorescence read out. Image recording time and signal to background ratio of single molecule events could be substantially ameliorated by introducing an asynchronous image recording protocol, named PALM with independently running acquisition (PALMIRA) [47, 48]. The use of a single wavelength and a freely running CCD camera in this imaging scheme radically simplified single molecule based super-resolution microscopy.

Further advancements in the field comprised dual- [49, 50] and multicolor imaging [51, 52] as well as three dimensional imaging by optical sectioning achieved via two-photon activation [53]. Super-resolution localization along the axial direction was first implemented using cylindrical optics [54] and double-plane detection [55]. Also, SMS microscopy has proven to be compatible with live cell imaging as demonstrated by recordings of individual adhesion complexes [56] and by time-lapse imaging of the endoplasmatic reticulum of a mammalian cell [57]. By fusing the idea of switching single molecules with the conception of ground state depletion [27, 28], formerly a domain of RESOLFT microscopy, it was successfully demonstrated most recently that SMS microscopy can be realized utilizing ordinary organic fluorophores [58]. 


\section{The RSFP rsFastLime}

The invention of the microscope literally opened the window to a new world by allowing scientists to see and study things which had previously been concealed from their perception. An incident which turned out to have a similarly revolutionary impact on the development of science was the discovery of the green fluorescent protein (GFP) from the jellyfish Aequorea Victoria by O. Shimomura in 1962 [59]. Following the isolation of the GFP gene [60] and its first application as a fluorescence marker [61] it quickly developed into an indispensable tool in the life sciences. Being the first fully genetically encoded fluorescent probe available, it enabled the noninvasive imaging of reporter gene expression, protein trafficking and a multitude of biochemical processes inside living cells [62]. Importantly, the GFP chromophore is formed autocatalytically from a part of the protein peptide chain meaning that apart from molecular oxygen no cofactors are required for its formation [63]. Since the discovery of the green fluorescent protein a number of GFP-homologues have been found, many of which occur in the class of the anthozoans (sea anemones and corals) [64]. To date, the colors of available fluorescent proteins (FPs) cover almost the entire visible spectrum [65]. For their groundbreaking work in connection with the discovery of the green fluorescent protein and its development into a ubiquitous tool in the life sciences O. Shimomura, M. Chalfie and R.Y. Tsien were awarded the 2008 Nobel prize in Chemistry.

A variant of the classical FPs are the so-called photoactivatable FPs. These are fluorescent proteins which may be irreversibly switched from a dark to a bright state or from one color to another, usually via illumination with a wavelength other than the excitation light [66, 40]. Yet another class of FPs are the reversibly switchable fluorescent proteins (RSFPs), which can be repeatedly transferred from a dark to a bright state and back. The most prominent RSFPs are the red emitting asFP595 $\left(\lambda_{e m}=595 \mathrm{~nm}\right)$ from the sea anemone Anemonia sulcata [67] and the green fluorescing Dronpa $\left(\lambda_{e m}=522 \mathrm{~nm}\right)$ from the coral Pectiniidae [38]. The possibility to reversibly switch fluorescent markers on and off provides many opportunities for novel protein tracking applications. On top of that, RSFPs naturally lend themselves to be used as switchable labels in super-resolution fluorescence imaging [68]. As such they bear the superb potential of combining the advantages of highly specific and non-invasive in vivo labeling with the opportunity to explore cellular structures and processes in living specimens at nanometer resolution. The study of the applicability of the RSFP rsFastLime, a Dronpa derivative, to subdiffraction fluorescence microscopy is the subject of this chapter. It is demonstrated that in principle one and the same switchable marker can be employed for both ensemble- and single molecule based super-resolution imaging, underscoring that photoswitching constitutes the basic concept underlying all fluorescence nanoscopy techniques [5]. 
a

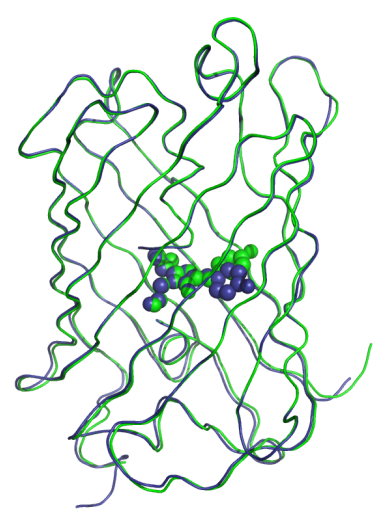

b

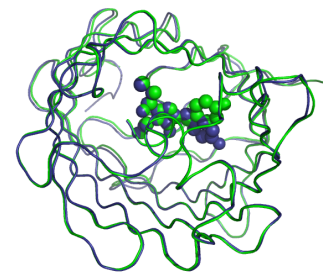

Figure 2.1: Overlay of the Dronpa on- and off-structure (on: green, off: blue). The chromophore atoms are shown as spheres and the protein backbones as thin tubes. Like all fluorescent proteins Dronpa and rsFastLime exhibit a GFP-like fold, namely an 11-stranded $\beta$-barrel enclosing an $\alpha$-helix which contains the autocatalytically formed chromophore. $\mathbf{a}$ and $\mathbf{b}$ display the same structures in two orthogonal views. The figure is courtesy of M. Andresen and A.C. Stiel.

\section{Structure and Switching Mechanism}

rsFastLime is another name for the mutant Dronpa-V157G of the RSFP Dronpa with which it shares most structural and photophysical properties. However, compared to Dronpa wild type rsFastLime features improved photoswitching characteristics, namely higher photostability and faster switching kinetics [69]. Under equilibrium conditions it resides in its fluorescent state. When excited with blue light $(\lambda \approx 490 \mathrm{~nm})$ it emits fluorescence peaked at $518 \mathrm{~nm}$ and after prolonged blue illumination is switched to the dark state. This process may be reversed by subjecting the protein chromophore to illumination with ultra violet light which transfers the molecule back to its fluorescent form. For single Dronpa molecules it was demonstrated that this on- and off switching could be repeated up to $\approx 100$ times [70].

The tertiary structure of Dronpa is shown in figure 2.1. It may also serve as a model for the very similar structure of rsFastLime. Like all fluorescent proteins, Dronpa and rsFastLime feature a GFP-like fold consisting of an 11-stranded $\beta$-barrel (also referred to as $\beta$-can) enclosing an $\alpha$-helix. Within the latter lies the autocatalytically formed chromophore which is made up of an imidazolinone 5-ring connected to a p-hydroxyphenyl 6-ring via a methine bridge. X-ray crystallography revealed that the on-state chromophore adopts a planar cis-conformation within the $\beta$-can [71, 72]. Additionally, the stabilizing effect of neighboring amino acids contributes to the high fluorescence quantum yield of the on-state chromophore $(\approx 0.85)$ as it efficiently impedes non-radiative dissipation of excitation energy. Upon excitation with blue $(\lambda \approx 490 \mathrm{~nm})$ light the chromophore may switch from the fluorescent to the non-fluorescent state by undergoing a cis-trans isomerization about the methine bridge. The process is accompanied by the rearrangement of several nearby residues. An illustration of the bright- and the dark state chromophore is shown in figure $2.2 \mathrm{a}$. It descriptively reveals the structural rearrangement taking place during photoisomerization. The 

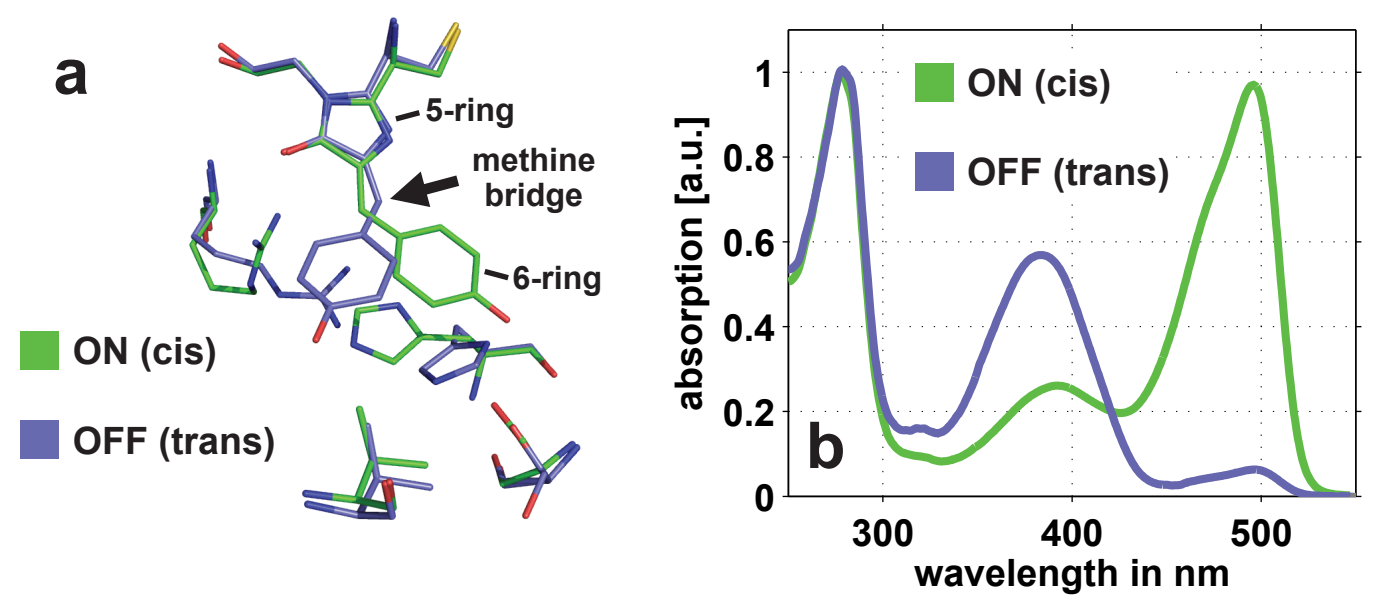

Figure 2.2: a Detailed comparison of the Dronpa chromophore in the on- and in the off-state and differences in their vicinities. Chromophores and residues are displayed as sticks, color-coded by atom type. On-state carbon: green, off-state carbon: light blue, oxygen: red, nitrogen: dark blue, sulfur: yellow. The key element in the switching process is a cis-trans photoisomerization about the methine bridge connecting the 5- and the 6-ring of the chromophore; it is accompanied by changes in nearby residues and in the hydrogen-bonding network. b absorption spectra recorded on purified rsFastLime protein in solution maximally switched to the on-state (green) or to the off-state (blue). Illumination of the on-state protein near its absorption maximum at $496 \mathrm{~nm}$ strongly reduces absorption at that wavelength while elevating the absorption peak at $395 \mathrm{~nm}$. This process is reversed when illuminating the off-state protein near its absorption maximum at $395 \mathrm{~nm}$. The figure is courtesy of M. Andresen and A.C. Stiel.

absorption spectra corresponding to the two isomerization states are depicted in $2.2 \mathbf{b}$. They were recorded on purified rsFastLime protein in a buffered solution at $\mathrm{pH} 7.4$ maximally switched to either the on- (green) or the off state (blue). In the fluorescent cis-configuration (green), molecules feature a main absorption peak at 496nm and a smaller second maximum at $395 \mathrm{~nm}$. After switching the protein to the dark state via prolonged irradiation near the $496 \mathrm{~nm}$ band absorption at that wavelength is strongly reduced while the peak at $395 \mathrm{~nm}$ increases (blue). Thermal relaxation from the non-fluorescent to the fluorescent form takes place on a time scale of several hours [72]. However, this process can be dramatically enhanced through illumination with ultra violet light.

\subsection{Reversible Switching at the Ensemble- and Single Molecule Level}

In order to characterize the photoswitching properties of rsFastLime, a number of spectroscopic measurements were carried out. The setup on which these experiments were performed is displayed in figure 2.3. The light from a blue (488 or $491 \mathrm{~nm})$ and from a ultra violet (403 or $416 \mathrm{~nm}$ ) laser was overlaid using a dichroic mirror and focused into the sample with an oil immersion objective lens to yield two superimposed homogeneous spots of 


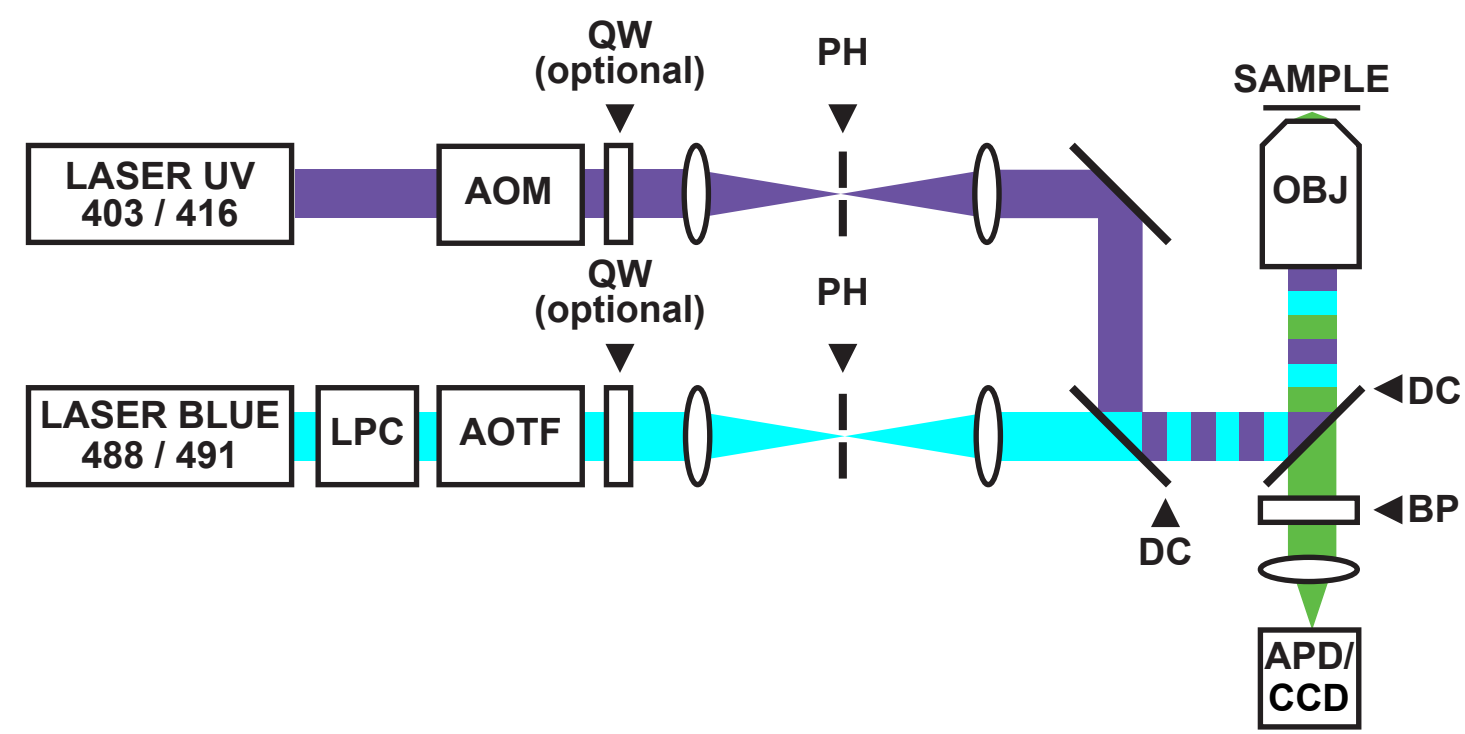

Figure 2.3: Setup used for spectroscopic measurements of the RSFP rsFastLime. LPC: laser power controller, AOM: acousto-optical modulator, AOTF: acousto-optical tunable filter, QW: quarter waveplate, PH: pinhole, DC: dichroic mirror, OBJ: objective lens (oil immersion, 100x, NA 1.4), BP: bandpass filter, APD: avalanche photodiode, CCD: charge coupled device camera.

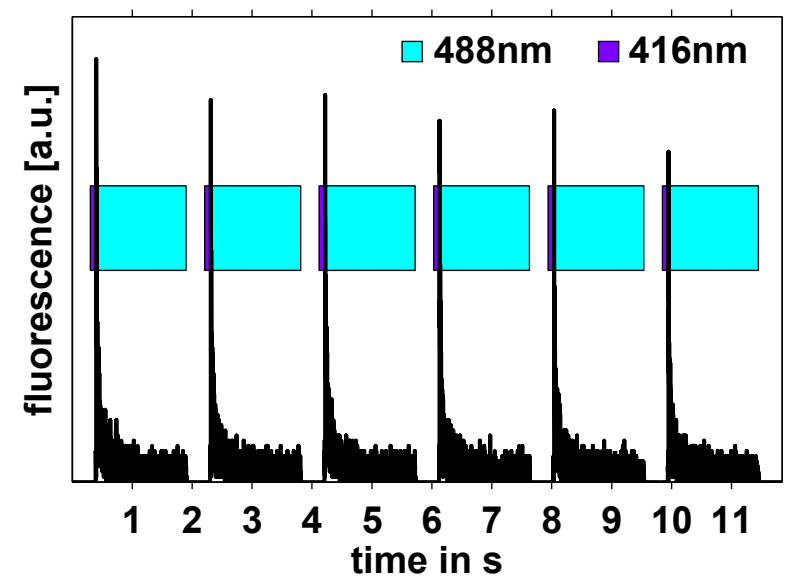

Figure 2.4: Reversible photoswitching of an ensemble of rsFastLime molecules in a film of polyvinyl alcohol (PVA). The colored bars indicate the timing of the successively applied 488nm (turquoise) and 416nm (purple) illumination light. Illumination at $488 \mathrm{~nm}\left(\Delta t=1.5 \mathrm{~s}, I_{488}=200 \mathrm{~W} / \mathrm{cm}^{2}\right)$ elicits fluorescence (emission maximum at $518 \mathrm{~nm}$ ) and concomitantly switches the protein to a long-lived dark state, indicating that the dark state is populated via the first excited singlet state $S_{1}$. Fluorescence can be recovered via illumination with $416 \mathrm{~nm}$ activation light $\left(\Delta t=0.1 \mathrm{~s}, I_{416}=50 \mathrm{~W} / \mathrm{cm}^{2}\right)$. The overall decrease in the fluorescence signal over several switching cycles can be attributed to irreversible photobleaching of the chromophore. 
typically $3-10 \mu \mathrm{m}$ in diameter. The exact size of the the illumination spots could be tuned by varying the size of the pinholes ( $\mathrm{PH})$ and/or the focal lengths of the lenses behind the pinholes. Fluorescence light was collected through the same objective lens, separated from the excitation light using another dichroic mirror and focused alternatively on an avalanche photo diode (APD) or on a CCD camera. Whereas the APD was used for measurements that required high time resolution (up to 250ns), the CCD mainly served the characterization of single molecule blinking. Laser power was controlled via an acousto optical tunable filter (blue) and an acousto optical modulator (UV). The use of a quarter wave plates (QW) allowed to change the polarization from linear to circular.

If rsFastLime was to be employed as a marker molecule in a RESOLFT-type microscope it had to be demonstrated that i) it could be reversibly switched between its bright state $S$ and its dark state $D$ at the ensemble level and ii) the light-driven transition from the on- to the off-state was saturable in the sense discussed in chapter[1[14].

The feasibility of i) is displayed in figure 2.4. The figure shows the fluorescence signal emitted by an ensemble of the purified protein rsFastLime immobilized in a thin film (thickness $\approx 100 \mathrm{~nm}$ ) of the polymer polyvinyl alcohol (PVA) upon repeated successive illumination with 488 - and $416 \mathrm{~nm}$ light. With the protein in its bright state $S$, excitation at $488 \mathrm{~nm}$ at an intensity of $I_{488}=200 \mathrm{~W} / \mathrm{cm}^{2}$ (see turquoise color bar) elicits a fluorescence signal which after an illumination time of $\Delta t_{488}=1.5 \mathrm{~s}$ decays to approximately $2 \%$ of its initial value. This gradual decay of the emitted fluorescence can be attributed to the switching of the majority of molecules from the fluorescent on-state $S$ to the dark state $D$. The following pulse of $416 \mathrm{~nm}$ activation light at an intensity of $I_{416}=50 \mathrm{~W} / \mathrm{cm}^{2}$ (purple color bar) and a duration of $\Delta t_{416}=0.1 \mathrm{~s}$ almost completely restores the bright state population to its previous value, as evidenced by the fluorescence signal created during the next $488 \mathrm{~nm}$ excitation pulse. This on- and off-switching can be repeated several times.

Already from this first simple experiment a number of conclusions can be drawn regarding the nature of the photoswitching process and the use of rsFastLime as a label in RESOLFT microscopy. As is evident from figure 2.4, the $488 \mathrm{~nm}$ excitation light not only serves the generation of the fluorescence signal but at the same time induces the transition of the protein chromophore to its dark state $D$. This observation strongly suggests that the on- to off-state transition occurs via the first excited singlet state $S_{1}$, implying that the fluorescence transition $S_{1} \rightarrow S_{0}$ and the transition to the dark state $S_{1} \rightarrow D$ constitute two competing processes: once excited from $S_{0}$ to $S_{1}$ by $488 \mathrm{~nm}$ light, a single rsFastLime chromophore either returns to the singlet ground state $S_{0}$ via emission of a fluorescence photon (or non-radiative de-excitation) or switches to the non-fluorescent dark state by undergoing a conformational rearrangement (neglecting the possibility of photobleaching from $S_{1}$ or promotion to higher excited states). Consequently, the number of photons radiated off by a single marker before it is switched off is limited by the quantum yield $\phi_{\text {off }}$ of the $S_{1} \rightarrow D$ transition. For the use of rsFastLime and (similar RSFPs) as a label in a RESOLFT-type microscope this is an important conclusion as the underlying mechanism fundamentally restricts the fluo- 

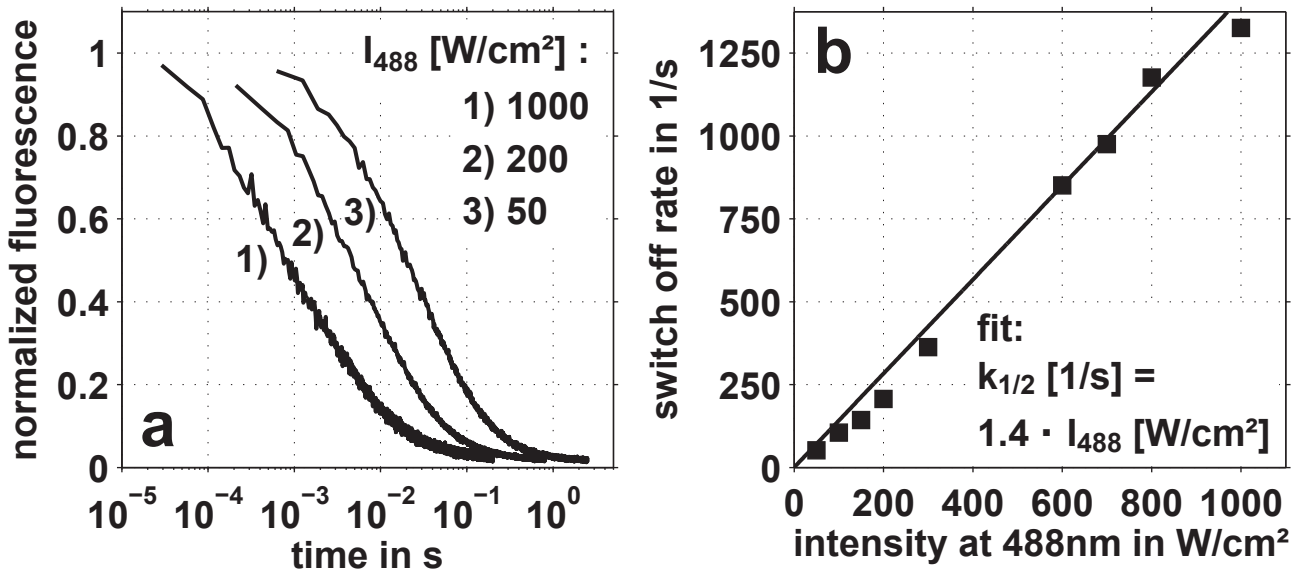

Figure 2.5: Switching an ensemble of rsFastLime in PVA to the dark state at different intensities of the 488nm excitation/switch-off light. a Characteristic fluorescence decay for three different switch-off intensities. All curves are normalized to the signal recorded at time $t=0$. At higher intensities switching occurs on shorter time scales. Notably, the switch-off efficiency (i.e. the residual fluorescence level after switch-off) does not markedly change when varying the intensity, indicating that both transitions on $\rightleftarrows$ off are light-driven. b Switch-off rate $k_{1 / 2}=1 / \tau_{1 / 2}$ as a function of the switch-off intensity $I_{488} \cdot \tau_{1 / 2}$ denotes the time at which fluorescence has decayed by 50 percent. The linear dependence of $k_{1 / 2}$ on the applied intensity suggests that the light driven transitions are first order processes.

rescence signal which can be obtained during one switching cycle. It should be noted that this is in contrast to other RSFPs such as e.g. asFP595. The latter may in principle emit an unlimited number of fluorescence photons before being switched off by light of a different wavelength [68]. Rather, the switching scheme found in rsFastLime is somewhat reminiscent of the transition $S_{1} \rightarrow T_{1}$ to the dark triplet state, the mechanism underlying Ground State Depletion (GSD) microscopy [27, 28]. Neglecting processes other than photoswitching and fluorescence emission, the number of photons emitted per switching cycle is described by a geometric distribution

$$
p(N)=\phi_{\text {off }}\left(1-\phi_{\text {off }}\right)^{N}, \quad \bar{N}=\frac{1-\phi_{\text {off }}}{\phi_{\text {off }}}
$$

where $p(N)$ is the probability of a molecule to emit $N$ photons in one switching cycle and $\bar{N}$ is the mean number of photons emitted per cycle.

The characteristic time scale on which the $S_{1} \rightarrow D$ transition takes place depends on the frequency at which molecules are cycled between the singlet states $S_{0}$ and $S_{1}$ and can thus be controlled by varying the intensity, i.e. the photon flux, of the applied $488 \mathrm{~nm}$ excitation light. This is of great practical relevance to the imaging process as it allows to speed up image acquisition by choosing higher excitation intensities. The connection between switching speed and excitation intensity is illustrated in figure 2.5. As an example figure 2.5a features the fluorescence signal emitted by an ensemble of rsFastLime molecules in PVA upon irradi- 
ation at $488 \mathrm{~nm}$ at three different intensities. Clearly, excitation at a higher intensity results in faster photoswitching as documented by the different time scales of the fluorescence decay monitored at $I_{488}=50,200$ and $1000 \mathrm{~W} / \mathrm{cm}^{2}$. A more quantitative analysis of the switching speed as a function of the applied intensity is shown in figure $2.5 \mathbf{b}$ which features the switchoff rate $k_{1 / 2}=1 / \tau_{1 / 2}$ plotted versus the excitation intensity $I_{488}$. Here, $\tau_{1 / 2}$ denotes the time after which the fluorescence signal has decayed to the average between its maximum and its minimum (i.e. equilibrium) value (maximum+minimum)/2. The linear dependence of the switching rate $k_{1 / 2}$ on the intensity underscores the assumption made previously that photoswitching from the on- to the off-state in rsFastLime is a first order process. This means that the cis-trans isomerization of the chromophore is initialized spontaneously from $S_{1}$ (or possibly from another intermediate state which can be populated from $S_{0}$ by excitation at around $488 \mathrm{~nm}$ ) and does not require additional excitation to higher excited intermediate states.

Another important observation is that at the ensemble level fluorescence cannot be switched off entirely. Even after long irradiation at $488 \mathrm{~nm}$ fluorescence continues to be emitted, with the signal after switch-off amounting to $\approx 1 \%$ of the value recorded at the onset of the $488 \mathrm{~nm}$ illumination (i.e. before switch-off). In the following, the fluorescence signal after switch-off in units of the signal measured before switch-off shall be referred to as residual fluorescence. It may be pointed out that the effect of an incomplete switch-off is in congruence with the dark state absorption spectrum of figure 2.2 which illustrates that even after maximal irradiation at $488 \mathrm{~nm}$ an ensemble of rsFastLime molecules still exhibits non-zero absorption at around 490nm. Interestingly, the residual fluorescence level does not markedly change over a wide range of values of the excitation intensity: For values of $I_{488}$ ranging from few W/cm ${ }^{2}$ up to several $\mathrm{kW} / \mathrm{cm}^{2}$ it stays constant at $\approx 1 \%$, as can be seen in figure 2.5 .

A priori there are two possible explanations for this phenomenon: i) A fraction of $\approx 1 \%$ of all proteins are fluorescent but cannot be switched to the dark state due to e.g. incomplete folding; in this case, the observed residual fluorescence stems from this non-switchable fraction. ii) $488 \mathrm{~nm}$ light not only elicits fluorescence and switches molecules to the dark state but with a small probability reverses the cis-trans isomerization of the rsFastLime chromophore, activating it from the dark state $D$ back to the bright state $S$.

The simplest way to determine which of the aforementioned scenarios applies is to monitor the fluorescence emission trajectories of single molecules under constant $488 \mathrm{~nm}$ illumination. In this experiment the existence of a fraction of non-switchable proteins (scenario i)) would result in appreciably longer on-times of these molecules as compared to those which are switched to the dark state (finally, also the non-switchable proteins will turn off when undergoing photobleaching). A clear signature of scenario ii) (switching-on by $488 \mathrm{~nm}$ light) would be the repeated on-and off blinking of one and the same emitter with comparably long off-times during which it resides in the dark state. It should be noted, though, that the blinking of single molecules alone does not serve as prove that the transition from the darkback to the fluorescent state is induced by the $488 \mathrm{~nm}$ excitation light, as this could likewise be explained by spontaneous (thermal) relaxation from $D$ to $S$. However, at the ensemble level the latter would manifest itself as a dependence of the residual fluorescence level on $I_{488}$, in contrast to what is observed.

To test these two hypotheses (non-switchable fraction vs on- and off-switching by $488 \mathrm{~nm}$ 

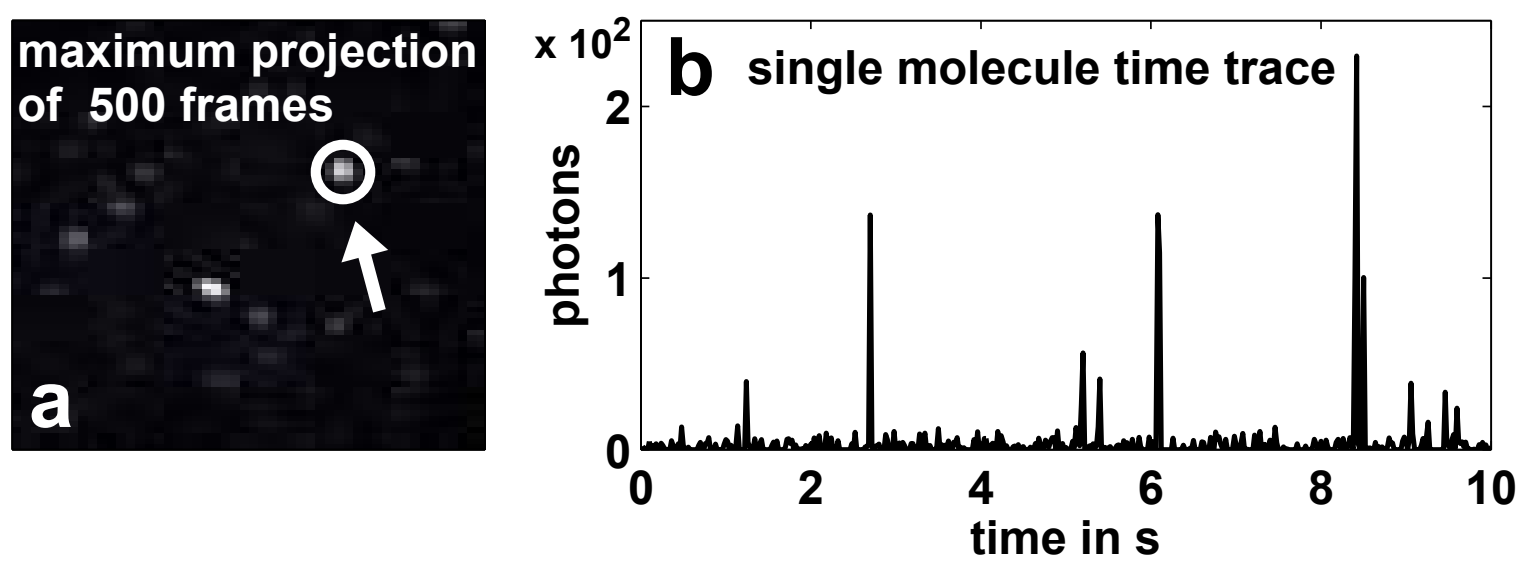

Figure 2.6: Evidence for the repeated cycling of single rsFastLime molecules between the fluorescent- and the non-fluorescent state under $488 \mathrm{~nm}$ illumination. a The maximum projection of a series of 500 camera frames demonstrates that the sample is made up of individual rsFastLime molecules. b Fluorescence time trace of the single rsFastLime molecule highlighted in a), featuring fluorescence bursts of millisecond duration and long off-times in the order of seconds. Higher illumination intensities result in shorter off-times (not shown) of single molecules, suggesting that $488 \mathrm{~nm}$ illumination also serves the activation of rsFastLime from the darkto the fluorescent state. The data is courtesy of C. Geisler and A. Egner.

light) a dilute sample of rsFastLime molecules embedded in a thin film of PVA was prepared, irradiated with 488nm light and the emitted fluorescence detected on an electron multiplying charge coupled device (EM-CCD) camera. The protein concentration was chosen low enough to make the probability of finding more than one of them in the same diffractionlimited area negligible. This way it was ensured that fluorescence emitted from the same position in the sample in different camera frames originated from one and the same emitter. Figure 2.6 shows a typical fluorescence time trace of a single rsFastLime molecule. At the excitation intensity chosen the protein exhibits repeated on- and off blinking with millisecond on-times and off-times in the range of seconds. Several hundreds of monitored rsFastLime molecules showed similar blinking behavior, strongly suggesting that irradiation at $488 \mathrm{~nm}$ also activates molecules from the dark to the fluorescence state. Evidence for a non-switchable fraction of molecules featuring significantly longer on-times was not found.

Based on these experimental findings, a preliminary photophysical model of the RSFP rsFastLime can be established, depicted in figure $2.7 \mathrm{a}$. Illumination with $488 \mathrm{~nm}$ light excites the chromophore from the singlet ground state $S_{0}$ to the first excited singlet state $S_{1}$. Once in $S_{1}$ it may either return to $S_{0}$ emitting a fluorescence photon or undergo a conformational rearrangement (cis-trans isomerization) and switch to the non-fluorescent dark state $D$. On the other hand, a molecule in the dark state $D$ can be (re-)activated to the fluorescent state via absorption of an ultra violet $(\lambda \approx 400 \mathrm{~nm})$ or a blue $(\lambda \approx 490 \mathrm{~nm})$ photon. Most probably, the activation process takes place via some intermediate excited state, denoted $D^{*}$ in the diagram [70]. Figure 2.7b shows a simplified version of the same model in which the intermediate 


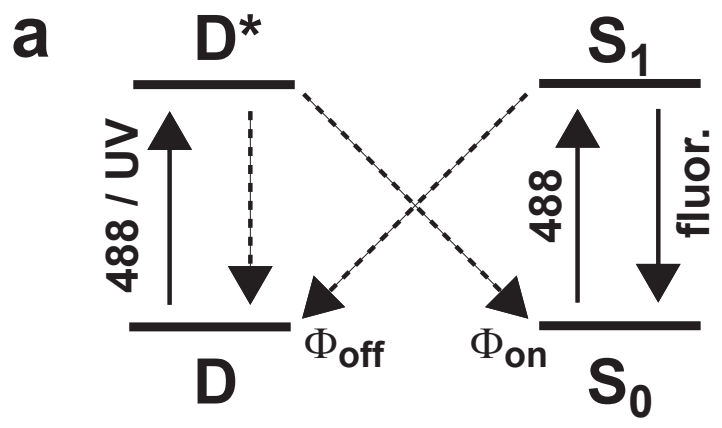

b

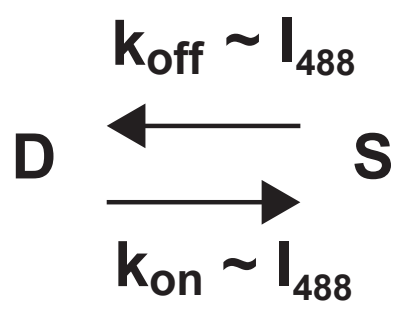

Figure 2.7: Preliminary photophysical model of the RSFP rsFastLime. Dashed lines denote non-radiative transitions. a $488 \mathrm{~nm}$ light excites the molecule from $S_{0}$ to the first excited singlet state $S_{1}$. From $S_{1}$ it either returns to $S_{0}$ emitting a fluorescence photon or, with a probability $\phi_{\text {off }}$, switches to the dark state $D$ via a conformational rearrangement of the chromophore. A molecule in the dark state $D$ can be switched back to $S_{0}$ via an intermediate state $D^{*}$. Activation from $D$ to $S_{0}$ via $D^{*}$ can be induced effectively by light at around $400 \mathrm{~nm}$ and less effectively by light at around 490nm (at the ensemble level only $\lambda \approx 400 \mathrm{~nm}$ serves activation). b Simplified photophysical model of rsFastLime. The on- to off transition $S \rightarrow D$ as well as the activation transition $D \rightarrow S$ are one-photon processes. Consequently, the equilibrium population of the fluorescent state $S$ under sole $488 \mathrm{~nm}$ irradiation is independent of the applied intensity.

states $S_{1}$ and $D^{*}$ are merged with the ground states $S_{0}$ and $D$, respectively, to form one fluorescent state $S$ and one dark state $D$. Yet the simplified model emphasizes the main features of rsFastLime as far as its use as a RESOLFT-marker is concerned, namely the possibility to reversibly switch the chromophore between a fluorescent- and a non-fluorescent state via one photon processes induced by blue and UV light.

For matters of completeness it shall be added that the switching-on of rsFastLime from the dark- to the bright state can be induced thermally, too: due to thermal relaxation an ensemble of rsFastLime molecules not previously subjected to illumination is in the fluorescent state. However, at room temperature this process occurs on a time scale of hours [72] and is therefore of no relevance to the imaging process which relies on light-induced switching on millisecond- to second time scales. For this reason thermal activation has not been included in the photophysical model illustrated in figure 2.7 .

At this point it is most vital to stress again that at the ensemble level only ultra violet light serves the activation of rsFastLime to the fluorescent state. Although a single rsFastLime molecule in the dark state may be excited by blue light (i.e. $\lambda \approx 490 \mathrm{~nm}$ ) as demonstrated in figure 2.6, the effective cross section for the reverse process $S \rightarrow D$ at this wavelength is larger by approximately two orders of magnitude. As a consequence, in a bulk measurement illumination with $488 \mathrm{~nm}$ light always entails a decay of the fluorescence signal until the equilibrium between molecules in $D$ and $S$ is reached. The equilibrium value of the fraction of chromophores in the fluorescent state, denoted $r$, is determined by the values of 
the effective cross sections for the on- and the off transition:

$$
r=\frac{\sigma_{o n}}{\sigma_{\text {on }}+\sigma_{\text {off }}} \approx 0.01
$$

This simple insight is of considerable importance. It states that when subjected to $488 \mathrm{~nm}$ irradiation only a small fraction of rsFastLime molecules are emitting fluorescence at a time while the majority remain non-detectable. As individual emitters are being switched on and off perpetually, the random subset of chromophores in the fluorescent state changes continuously. Hence, after a sufficiently long period of time virtually every molecule has been transferred to the on-state and back at least once, enabling the stochastic sampling of the complete set by successive switching of individual emitters. It is this property that allows the use of rsFastLime as a marker in single molecule based localization microscopy, which will be discussed in a later section.

\subsection{RESOLFT Microscopy with rsFastLime}

In this section it shall be demonstrated that the switching mechanism found in rsFastLime allows for its use as a marker in RESOLFT microscopy. After the reversibility of the switching process has been exemplified in the preceding section it remains to be shown that at least one of the transitions $S \leftrightarrows D$ can be driven into saturation. On the basis of the preliminary two-state model depicted in figure 2.7 it can be concluded that both the on- as well as the off-transition meet this condition (compare figure 1.2 and the subsequent discussion). Depending on which transition is utilized there are two imaging modes which are, in principle, equally suited for super-resolution RESOLFT microscopy with rsFastLime. These are 1) saturated depletion of the fluorescent state, denoted positive imaging and 2) negative imaging, which may refer to the saturated depletion of the dark state. Figure 2.8 depicts a single step of the image acquisition process for both imaging modes, showing the illumination sequence applied at one particular position of the intensity-modulated pattern with respect to the sample. In the case of positive imaging the switch-off light is intensity modulated leaving subdiffraction-sized fluorescent spots at the intensity zeros of the switch-off beam. The situation is reversed in negative imaging: here, the switch-on beam is intensity modulated giving rise to super-resolution-sized dark spots at the intensity naughts of the activation pattern. In both cases, scanning the sub- $\lambda$-sized spots over the sample and recording the fluorescence signal at each pattern position yields a super-resolution image of the sample structure.

There are reasons, however, which make the positive imaging approach more straight forward to implement: whereas in the positive mode the signal collected at each pattern position can be directly assembled to yield a super-resolution image of the specimen, negative imaging requires deconvolution and further computational processing. Furthermore, in the positive imaging mode super-resolution information is transferred with a higher signal-tonoise ratio. The latter can be seen when comparing the Fourier transform of the emission probability pattern (black lines in figure 2.8) in both cases [73]. It is for these reasons that only the positive imaging approach was pursued in this work. 


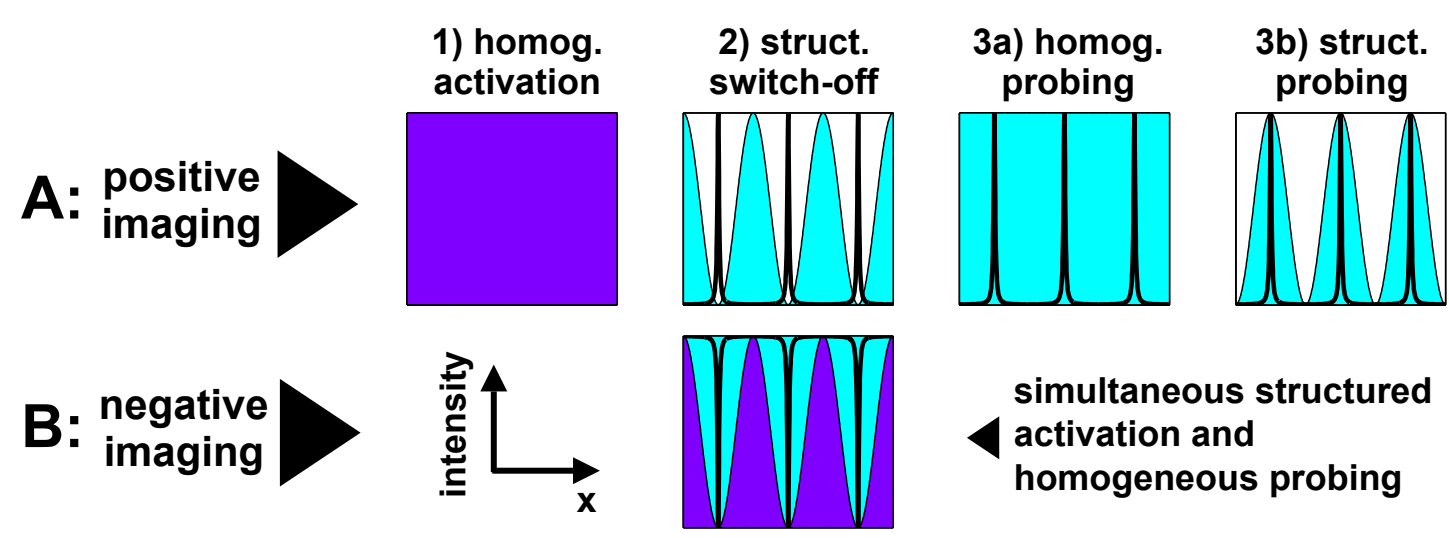

Figure 2.8: Two possible implementations of RESOLFT-microscopy with rsFastLime. Positive imaging (first row) : 1) Homogeneous illumination with UV light switches molecules to the fluorescent state. 2) Structured blue switch-off light featuring one or several zeros leaves only emitters in a subdiffraction-sized vicinity of the intensity naughts in the fluorescent state (black lines: emission probability after saturated switch-off). 3) Fluorescence of on-state markers is read out by 3a) homogeneous $O R$ 3b) structured blue probe light. Negative imaging (second row): simultaneous illumination with structured UV switch-on light and homogeneous blue light elicits fluorescence everywhere except in the subdiffraction-sized vicinity of the intensity naughts of the switch-on pattern (black lines: emission probability). - In either case, scanning the sample with subdiffraction sized bright or dark spots reveals subdiffraction information. Whereas in positive imaging the super-resolution image is directly formed by the fluorescence signal recorded at different scanning steps, constructing the superresolution image from the data acquired in the negative imaging mode requires deconvolution and further computational processing or the data. Also, the signal-to-noise ratio of the high resolution information is more favorable in the positive imaging mode.

In the following, the optimal parameters for an implementation of RESOLFT microscopy with rsFastLime in the positive imaging mode are determined. This includes 1) finding the right illumination time and -intensity for fast and efficient switching-on of rsFastLime by UV light and 2) a characterization of the fluorescence depletion (i.e. switch-off) mechanism as a function of the duration and intensity of the applied blue $(\lambda \approx 490 \mathrm{~nm})$ illumination. For this purpose, measurements were carried out in such a way as to mimic the pump-probe-type image acquisition protocol described in figure 2.8 .

\section{Fluorescence Activation (Switching-on)}

Figure 2.9 shows the switching-on of rsFastLime from the dark state $D$ to the fluorescent state $S$ by ultra violet $(403 \mathrm{~nm})$ light of different intensity and pulse duration as measured in a pump-probe type scheme. The fluorescence time trace of a single measurement is illustrated in Figure $2.9 \mathrm{a}$. The ratio of the signals elicited by the second and the first probe pulse yields the fraction of emitters which, after complete switch-off, have been switched back to the fluorescent state $S$ by the second $403 \mathrm{~nm}$ pulse. In figure $2.9 \mathrm{~b}$ this on-state fraction is plotted versus the intensity $I_{\text {on }}$ of the second switch-on pulse, the three curves corresponding to different durations $\Delta t_{\text {on }}$ of this pulse. Notably, choosing shorter switch-on pulses results in a reduction of the switching-on efficiency. This is evidenced by the fact that only in the 

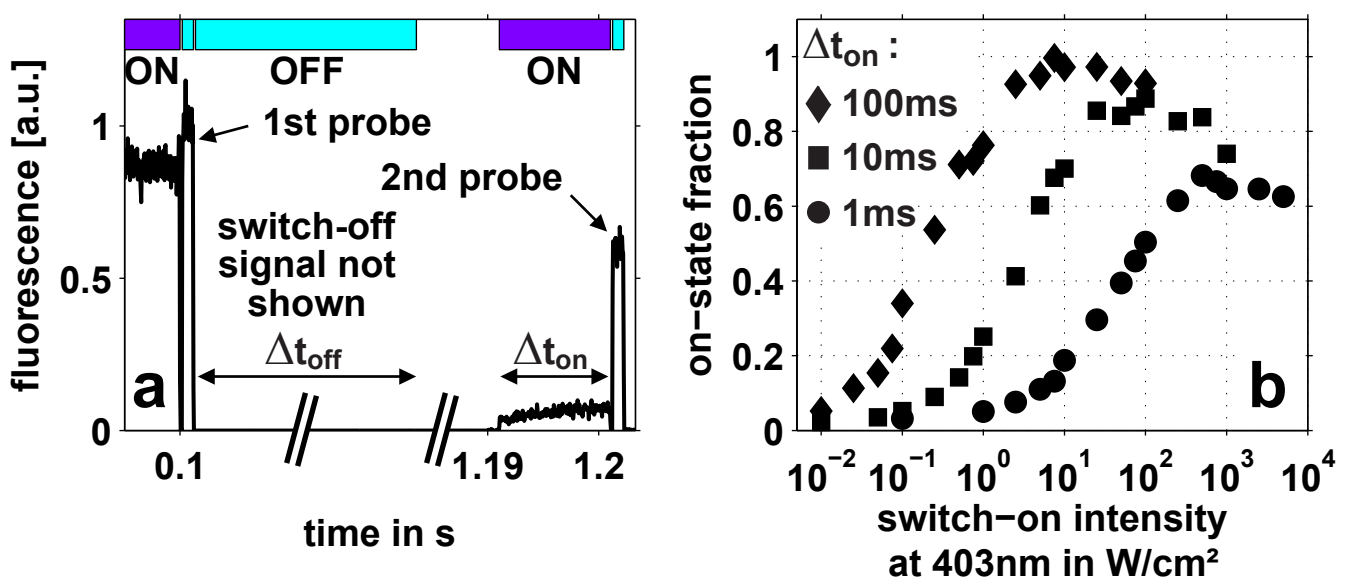

Figure 2.9: Switching-on of rsFastLime (purified, in PVA) by ultra violet $(403 \mathrm{~nm})$ light. a Exemplary fluorescence time trace of a single measurement performed in a pump-probe-type scheme. Color bars: timing of the applied illumination. From left to right: The sample is fully switched-on with $403 \mathrm{~nm}$ light $\left(I_{o n}=50 \mathrm{~W} / \mathrm{cm}^{2}, \Delta t_{\text {on }}=0.1 \mathrm{~s}\right.$, only the last $5 \mathrm{~ms}$ are shown; parameters of the first switch-on pulse are identical in all experiments) and the number of molecules in the on-state probed with $491 \mathrm{~nm}$ light $\left(I_{\text {probe }}=10 \mathrm{~W} / \mathrm{cm}^{2}, \Delta t_{\text {probe }}=1 \mathrm{~ms}\right)$. Following complete switch-off at $491 \mathrm{~nm}\left(I_{\text {off }}=100 \mathrm{~W} / \mathrm{cm}^{2}, \Delta t_{\text {off }}=1 \mathrm{~s}\right.$; corresponding signal not shown) another switch-on pulse is applied (variable duration and intensity) and the switched-on fraction probed (second probe pulse identical to the first). b On-state fraction (=2nd probe signal / 1st probe signal) vs switch-on intensity $I_{\text {on }}$ for three different values of $\Delta t_{\text {on }}\left(I_{o n}\right.$ and $\Delta t_{\text {on }}$ refer to the second switch-on pulse). The incomplete activation for short $\Delta t_{\text {on }}$ and high switch-on intensities $I_{o n}$ can be attributed to the population of a second short lived dark state and to photobleaching.

case of a pulse duration of $\Delta t_{o n}=100 \mathrm{~ms}$ all markers can be re-activated to the fluorescent state. For pulse durations of $\Delta t_{o n}=10 \mathrm{~ms}$ and $\Delta t_{o n}=1 \mathrm{~ms}$ only about 85 and 65 percent of all rsFastLime molecules, respectively, can be recovered from the dark state $D$. Furthermore, for each value of $\Delta t_{\text {on }}$ there is an optimal value of the switch-on intensity $I_{o n}$ at which activation from $D$ to $S$ is most efficient. The reason for the incomplete activation of rsFastLime for short switch-on pulses and high switch-on intensities predominantly lies in the population of a second short-lived dark state $\left(\tau_{1 / 2} \approx 50 \mathrm{~ms}\right)$ of the rsFastLime chromophore by the $403 \mathrm{~nm}$ switch-on light and is only partially caused by irreversible photobleaching: delaying the second probe pulse (rather than letting it follow the second switch-on pulse immediately) yields a higher value of the on-state fraction (not shown). However, due to the additional delay which is necessary to let molecules return from the short-lived dark state shorter switch-on times do not effectively speed up activation. The second dark state and its photophysics are discussed in more detail in a later chapter. As far as the imaging process is concerned, fast switching-on with high efficiency and minimal photobleaching is optimally carried out with the switch-on parameters $\Delta t_{o n} \approx 100 \mathrm{~ms}$ and $I_{o n} \approx 25 \mathrm{~W} / \mathrm{cm}^{2}$.

\section{Fluorescence Depletion (Switching-off)}

Figure 2.10 illustrates the saturation of the off-transition as recorded in a pump-probe mode, resembling the illumination scheme during image acquisition. While figure 2.10a depicts 

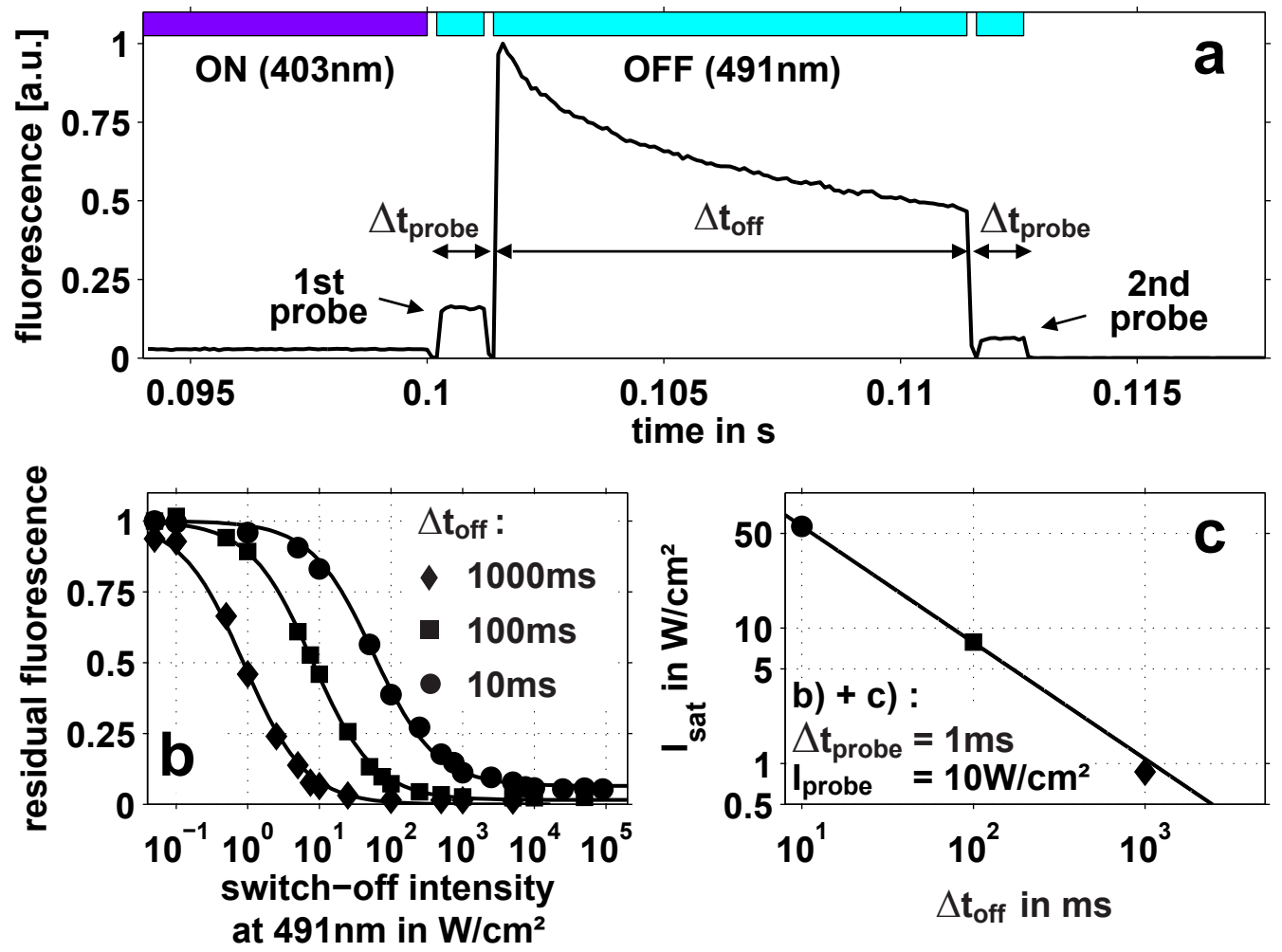

Figure 2.10: Saturation of the switch-off transition of rsFastLime (purified, in PVA); dependence of the switch-off curve on the duration of the switch-off pulse, $\Delta t_{\text {off. }}$ a Exemplary fluorescence time trace of a single measurement performed in a pump-probe-type scheme. Color bars: timing of the applied illumination. From left to right: The sample is fully switched-on with $403 \mathrm{~nm}$ light $\left(I_{o n}=50 \mathrm{~W} / \mathrm{cm}^{2}, \Delta t_{o n}=0.1 \mathrm{~s}\right.$, only the last $6 \mathrm{~ms}$ are shown) and the number of molecules in the on-state probed with $491 \mathrm{~nm}$ light $\left(I_{\text {probe }}=10 \mathrm{~W} / \mathrm{cm}^{2}, \Delta t_{\text {probe }}=\right.$ $1 \mathrm{~ms}$ ). Subsequently, a $491 \mathrm{~nm}$ switch-off pulse of varying duration and intensity is applied. Finally, a second probe pulse identical to the first measures the number of markers remaining in the fluorescent state after switchoff. b Residual fluorescence (second probe signal divided by first probe signal) vs $I_{\text {off }}$ for different values of $\Delta t_{\text {off }}$. Fits are according to equation (2.3). c $I_{\text {sat }}$ vs $\Delta t_{o f f}\left(I_{s a t}\right.$ is obtained from the fits in $\mathbf{b}$, see equation (2.3)). Longer switch-off pulses result in smaller saturation intensities, i.e. in a higher resolution at a given value of $I_{\text {off }}$.

the fluorescence time trace of an individual measurement figure $2.10 \mathrm{~b}$ features the residual fluorescence (second probe signal divided by first probe signal) plotted against the switch-off intensity $I_{\text {off }}$. Three of these switch-off curves are shown, each corresponding to a different duration $\Delta t_{\text {off }}$ of the switch-off pulse. Clearly, the off-transition exhibits a saturation behavior: for large enough intensities no dependence of the residual fluorescence on $I_{\text {off }}$ can be observed, furnishing experimental proof that rsFastLime is indeed suitable as a RESOLFT marker. Furthermore, comparing the different curves in figure $2.10 \mathrm{~b}$ leads to conclude that a longer switch-off time allows to attain a given depletion level at a lower intensity. Qualitatively, this result is in accordance with what is predicted by the two-state model shown in figure 2.7 (see also equation (1.4) ) and is a direct consequence of the negligible spontaneous recovery rate from the dark state $D$ to the fluorescent state $S$. Figure 2.10c puts this statement 

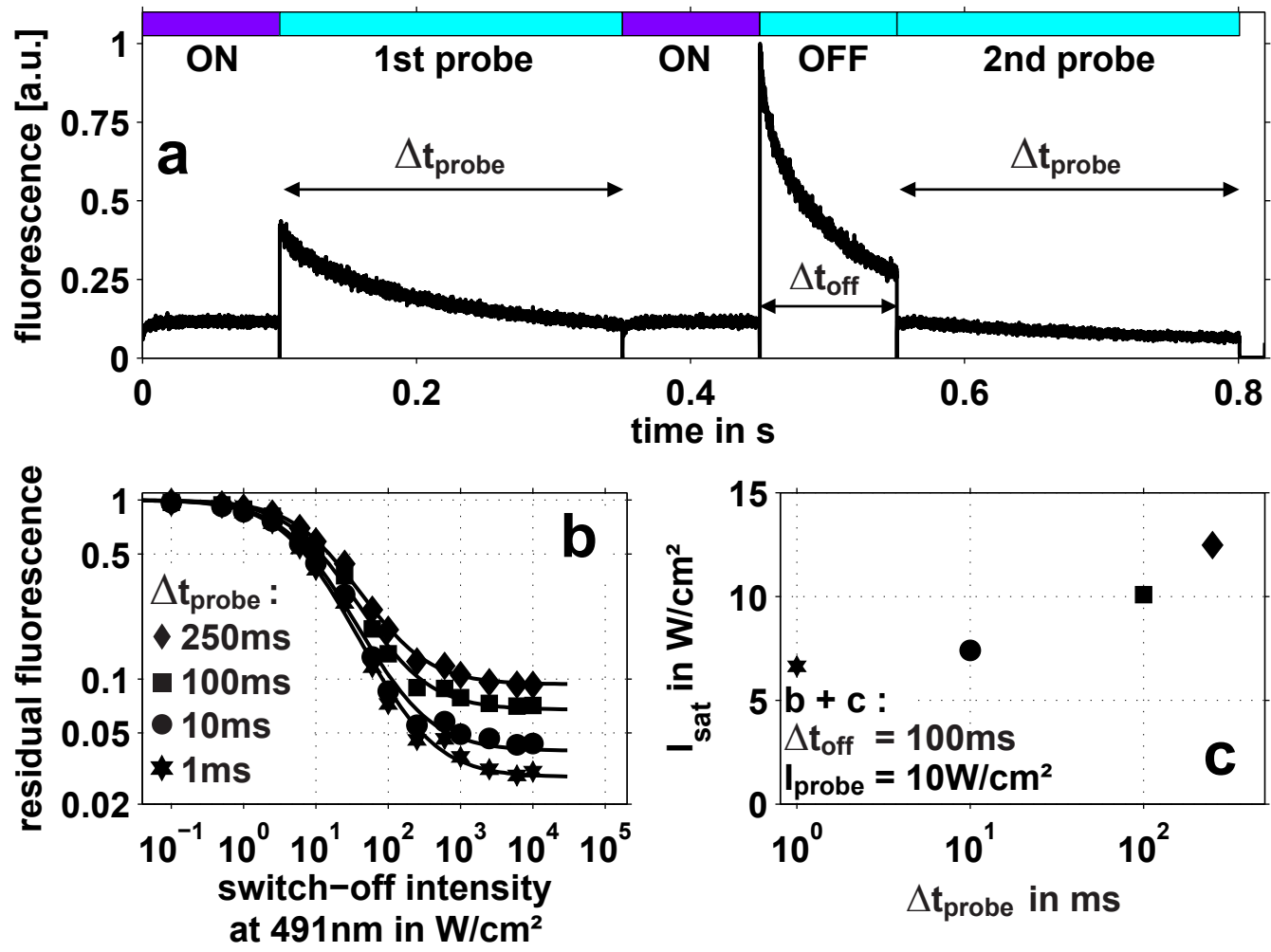

Figure 2.11: Saturation of the switch-off transition of rsFastLime (purified, in PVA); dependence of the depletion curve on the duration of the probe pulse, $\Delta t_{\text {probe }}$ a Exemplary fluorescence time trace of a single measurement performed in a pump-probe-type scheme. Color bars: timing of the applied illumination. From left to right: the sample is fully switched-on with $403 \mathrm{~nm}$ light $\left(I_{o n}=50 \mathrm{~W} / \mathrm{cm}^{2}, \Delta t_{o n}=0.1 \mathrm{~s}\right)$ and the number of molecules in the on-state probed with a $491 \mathrm{~nm}$ pulse of fixed intensity $\left(I_{\text {probe }}=10 \mathrm{~W} / \mathrm{cm}^{2}\right)$ but varying duration $\Delta t_{\text {probe }}$. After switching all markers back on with another $403 \mathrm{~nm}$ pulse identical to the first, a $491 \mathrm{~nm}$ switch-off pulse of fixed duration $\left(\Delta t_{o f f}=100 \mathrm{~ms}\right)$ but varying intensity is applied. Finally, a second probe pulse identical to the first measures the number of markers remaining in the fluorescent state after switch-off. b Residual fluorescence (=2nd probe signal / 1st probe signal) vs $I_{\text {off }}$ for different values of $\Delta t_{\text {probe }}$. Fits are according to equation (2.3). c $I_{\text {sat }}$ vs $\Delta t_{\text {probe }}\left(I_{\text {sat }}\right.$ is obtained from the fits in $\mathbf{b}$, see equation (2.3)). Longer probe times yield a higher signal but come at the expense of less efficient depletion and higher saturation intensities, i.e of a worsening of resolution at a given value of $I_{\text {off }}$. This is due to the fact that the probe pulse, too, switches molecules to the dark state.

in more quantitative terms featuring the saturation intensity $I_{\text {sat }}$ (see definition (1.6) plotted versus $\Delta t_{\text {off }}$; the curve depicts a power-law fit to the data (for further details and comments refer to the following section on experimental details). The key message contained in figure 2.10 c is that with rsFastLime as a RESOLFT marker it is possible to achieve unlimited resolution at arbitrarily low light intensities, as $I_{\text {sat }}$ can be made smaller simply by choosing a longer switch-off time $\Delta t_{\text {off. }}$.

Finally, figure 2.11 illustrates the effect of varying the duration of the probe pulse, 
$\Delta t_{\text {probe }}$, on the depletion curve and the attainable resolution. Figure 2.11 a shows an example of an individual measurement. Figure 2.11 b displays depletion curves recorded with probe pulses of varying duration; the corresponding plot of $I_{\text {sat }}$ versus $\Delta t_{\text {probe }}$ is depicted in figure 2.11c. While choosing a longer probe time yields a higher fluorescence signal this advantage is compromised by an effectively less efficient depletion of the fluorescent state and an increased saturation intensity, resulting in lower resolution at a given value of $I_{\text {off }}$. This is due to the fact that the probe pulse, too, switches molecules to the dark state $D$ (see figure 2.11a). Hence, longer probe times entail a lower contrast between the signal before and after switch-off.

Clearly, choosing the optimal probe pulse duration for super-resolution RESOLFT microscopy with rsFastLime means trading off image brightness versus resolution and should best be adapted to the labeling density of the sample.

\section{Experimental Details}

The function used to fit the depletion curves in figures $2.10 \mathrm{~b}$ and $2.11 \mathrm{~b}$ is given by

$$
\eta(I)=r+\frac{1-r}{1+I / I_{\text {sat }}}
$$

where $I, \eta(I), I_{\text {sat }}$ and $r$ denote, respectively, the switch-off intensity, the residual fluorescence, the saturation intensity and and the asymptotic residual fluorescence at high switch-off intensities. It is the $I_{s a t}$-values determined by these fits which are displayed in figures 2.10c and 2.11 c. The fit parameters corresponding to the different curves are listed in the following table:

\begin{tabular}{|c|c|c|c|c|}
\hline Figure & $\Delta t_{\text {off } f}[\mathrm{~ms}]$ & $\Delta t_{\text {probe }}[\mathrm{ms}]$ & $r$ & $I_{\text {sat }}\left[\mathrm{W} / \mathrm{cm}^{2}\right]$ \\
\hline $2.10 \mathbf{b}$ & 10 & 1 & 0.06 & 56 \\
\hline & 100 & 1 & 0.02 & 7.9 \\
\hline & 1000 & 1 & 0.005 & 0.9 \\
\hline $2.11 \mathbf{b}$ & 100 & 1 & 0.024 & 6.6 \\
\hline & 100 & 10 & 0.04 & 7.4 \\
\hline & 100 & 100 & 0.067 & 10 \\
\hline & 100 & 250 & 0.093 & 12 \\
\hline
\end{tabular}

Finally, the fit in figure $2.11 \mathrm{c}$ represents the function

$$
I_{\text {sat }}=410 \cdot \Delta t_{\text {off }}^{-0.86},
$$

where $I_{\text {sat }}$ is measured in $\mathrm{W} / \mathrm{cm}^{2}$ and $\Delta t_{\text {off }}$ in $\mathrm{ms}$.

Clearly, both the form (2.3) of the depletion curves as well as the dependence (2.4) of $I_{\text {sat }}$ on $\Delta t_{\text {off }}$ deviate from what is predicted by the two-state model shown in figure 2.7, suggesting that a more comprehensive model is required to satisfactorily describe the photophysics of rsFastLime. (The corresponding relations forecast by the two-state model are of the form $\eta(I)=r+(1-r) \exp \left(-I / I_{\text {sat }}\right)$ and $I_{\text {sat }} \propto 1 / \Delta t_{\text {off }}$; in particular $r$ is predicted to be independent of $\Delta t_{\text {off }}$, see equation 1.4 in chapter 11). Based on further experiments an extended model is established in a later section. 


\subsubsection{Imaging with rsFastLime}

Super-resolution RESOLFT microscopy based on the reversible switching of fluorescent proteins bears enormous potential as a tool in cell biology as it holds promise to combine the best of various techniques: the high specificity, non-invasiveness and 3D imaging capability of the fluorescence microscope; endogenous expression of the fluorescent marker inside living cells; and finally the ability of the RESOLFT microscope to image the specimen at subdiffraction resolution. On top of that the photo switching of fluorescent proteins can be carried out at light intensities which are several orders of magnitude lower than those required in STED- or even in GSD microscopy, thus keeping irradiation-induced damage of the specimen to a minimum. Indeed, it was shown in the previous section that using the RSFP rsFastLime as a marker in RESOLFT microscopy should enable super-resolution imaging at arbitrarily low light intensities. However, employing low switching intensities comes at the expense of prolonged image acquisition times. E.g., according to equation 2.4 . lowering the switch-off intensity by ten-fold requires an increase in the switch-off time by a factor of $10^{1 / 0.86} \approx 15$ if the same resolution is supposed to be achieved. As an example, $50 \mathrm{~nm}$ resolution with rsFastLime $\left(\lambda_{e m} \approx 520 \mathrm{~nm}\right)$ is equivalent to a resolution enhancement by approximately five-fold. If this is supposed to be realized with a switch-off intensity of $100 \mathrm{~W} / \mathrm{cm}^{2}$, the saturation intensity has to be equal to about $100 / 5^{2}=4 \mathrm{~W} / \mathrm{cm}^{2}$. According to equation 2.4 this can be achieved with a switch-off time of $\approx 215 \mathrm{~ms}$. Point-scanning of an area of only $1 \mu \mathrm{m} \times 1 \mu \mathrm{m}$ with these parameters would require a (minimum) recording time of $\approx 6$ minutes.

A simple means to speed up the imaging process without having to resort to higher switching intensities relies on parallelized data acquisition in a wide-field setup. Parallelization can be achieved by generating a multitude of subdiffraction-sized spots or lines at different positions in the sample at the same time and scanning them over the specimen. They may be produced by means of a structured (switch-off-) illumination pattern featuring several zero-intensity nodes or lines. The fluorescence emitted at different sample sites can be simultaneously recorded by imaging it onto a CCD camera in a wide-field detection scheme. The way in which a super-resolution image can be formed in spite of a diffraction-limited wide-field detection arrangement is discussed in a later paragraph.

\section{The Setup}

The setup used for parallelized wide-field RESOLFT microscopy with subdiffraction resolution in one lateral direction is shown in figure 2.12. It differs from the system presented in figure 2.3 only in that an iterferometric cavity of the Mach-Zehnder type for the generation of a structured switch-off pattern is added in the blue $(\lambda=488 / 491 \mathrm{~nm})$ beam path. Letting the beams in the two interferometer arms interfere at an angle $\alpha$ in the sample creates a standing wave along the lateral $\mathrm{x}$-direction. The intensity distribution of this standing wave can be described by $I \propto \sin ^{2}(\pi x / D)$, where $D=\lambda /(2 n \cdot \sin (\alpha / 2))$ is the distance between two adjacent maxima or minima, $\lambda$ the wavelength of the blue illumination light, $\alpha$ the interference angle and $\mathrm{n}$ the index of refraction. Thus, the periodicity $D$ can easily be controlled by altering the interference angle $\alpha$. The latter can be adjusted via tuning of the components in the interferometric cavity. In order to create perfect intensity zeros in the 


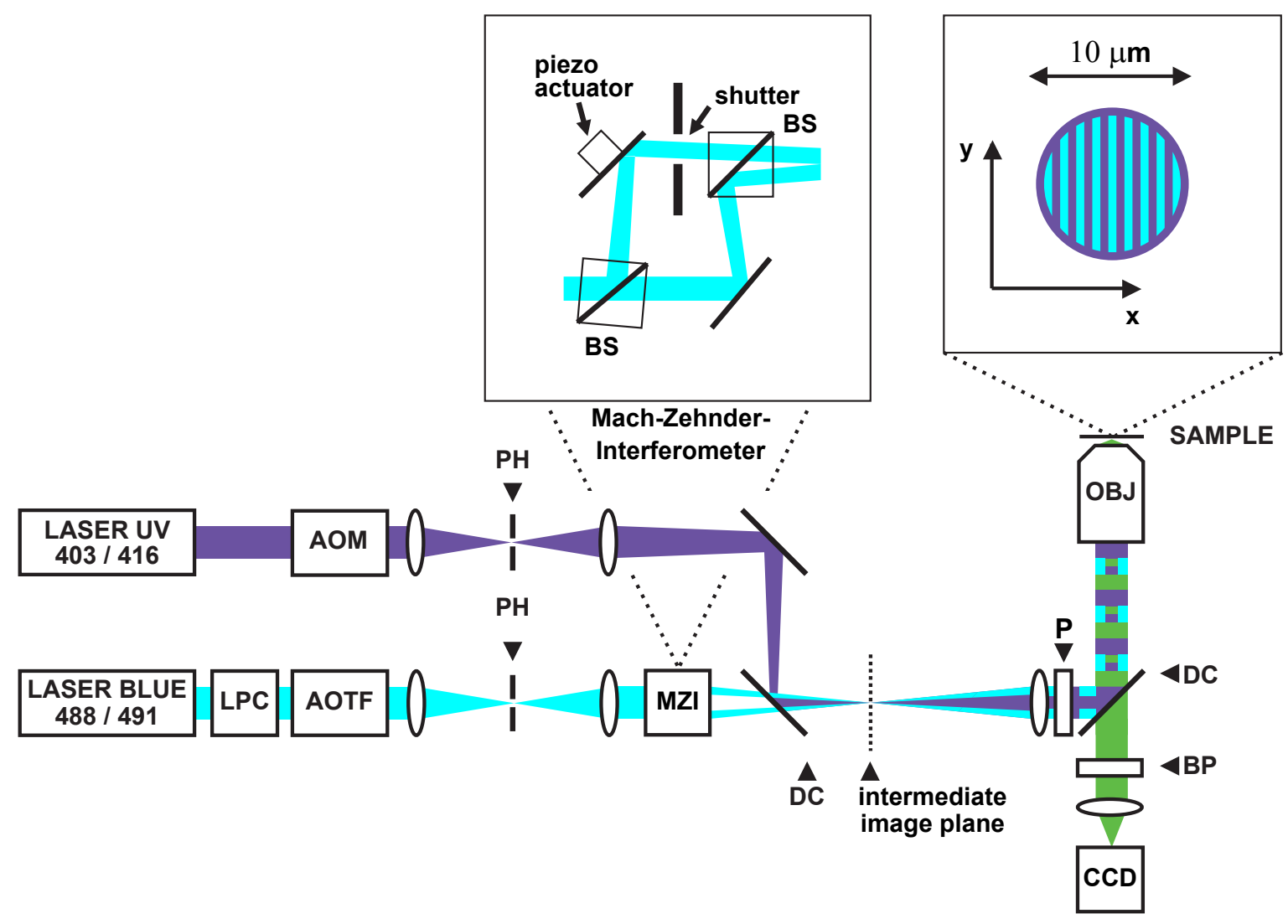

Figure 2.12: Setup used for parallelized wide-field RESOLFT imaging. The structured illumination pattern of the blue switch-off light is created by means of a Mach-Zehnder interferometer which splits the beam in two and recombines the two arms at a second beam splitter. By altering the angle between the interfering beams the periodicity of the resulting standing wave can be tuned. The pattern can be scanned over the sample by changing the relative phase of the interfering beams with a piezo-controlled mirror in one of the interferometer arms. Switch-off- and activation beam are superimposed in the sample focal plane to yield two overlaid spots of approximately $10 \mu \mathrm{m}$ in diameter. Fluorescence light is imaged onto a CCD camera. AOM: acousto optical modulator, LPC: laser power controller, AOTF: acousto optical tunable filter, PH: pinhole, DC: dichroic mirror, P: polarizer, BP: bandpass filter, BS: 50:50 non-polarizing beam splitter.

minima of the structured blue switch-off pattern it is essential that the two interfering beams be linearly polarized in the direction perpendicular to the plane spanned by the two beams, as a polarization component within this plane would result in a non-zero z-component. Finally, scanning the structured depletion light pattern over the sample along the lateral $\mathrm{x}$-direction can be achieved by altering the relative phase of the interfering beams. This is accomplished by controlling the position of one of the interferometer mirrors with a piezo actuator. The shutter in one of the interferometer arms allows to switch between structured and homogeneous blue illumination by selectively blocking the corresponding beam. Generating an image of the blue switch-off pattern in an intermediate image plane serves alignment purposes: mounting the CCD detector in this plane allows for direct observation and adjustment of the depletion light distribution. 

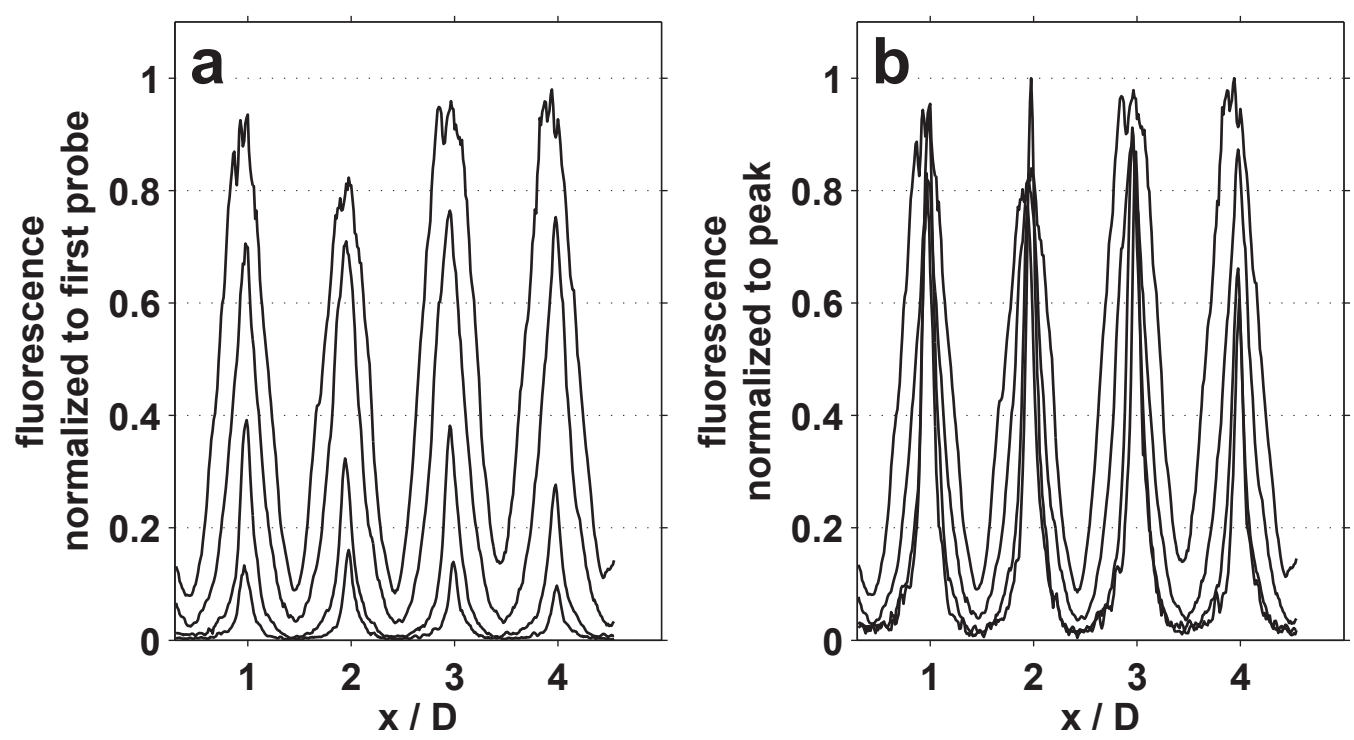

Figure 2.13: Narrowing of fluorescence peaks ( = increase in resolving power) with increasing switch-off intensity $I_{\text {off }}$ measured on a layer of rsFastLime (purified, in PVA). Illumination followed the scheme illustrated in figure $2.8 \mathrm{~A}, 3 \mathrm{~b}$ ) (structured probing). Parameters: $\Delta t_{\text {on }}=0.1 \mathrm{~s}, I_{\text {on }}=25 \mathrm{~W} / \mathrm{cm}^{2}, \Delta t_{\text {off }}=0.5 \mathrm{~s}, \Delta t_{\text {probe }}=10 \mathrm{~ms}$, $I_{\text {probe }}=10 \mathrm{~W} / \mathrm{cm}^{2}$. The applied switch-off intensities were, from top to bottom: $I_{\text {off }}=0.1,6,100$ and 750 $\mathrm{W} / \mathrm{cm}^{2}$, corresponding to $I / I_{\text {sat }}=0.053,3.2,53$ and 390. a Absolute value of fluorescence emission (arbitrary units). b Same data as in a normalized to the emission maximum of each curve, clearly indicating a narrowing of the stripes (i.e. an increase in resolution) at higher switch-off intensities. The decrease of the fluorescence signal as shown in a can be attributed to non-zero intensity minima of the switch-off light. The absolute distance between neighboring stripes was chosen as $D=3.66 \mu \mathrm{m}>>0.61 \lambda / N A=223 \mathrm{~nm}$ to minimize blurring caused by the detection optics.

\section{Characterization of the Resolving Power}

In the following the resolving power of the RESOLFT microscope displayed in figure 2.12 is characterized using the RSFP rsFastLime as a fluorescence marker. As compared to a conventional wide-field system the microscope features an enhanced resolution along the lateral $\mathrm{x}$-direction (see the inset in figure 2.12 for a definition of the $\mathrm{x}$-direction): saturating the depletion of the fluorescent state by means of the intensity-modulated blue switch-off light confines fluorescence emission to the vicinity of the zero-intensity lines of the depletion pattern. Along the x-direction the extension of these stripes can be reduced to subdiffraction size (see figure 2.8A). By contrast, the resolving power along the $\mathrm{y}$ - and the $\mathrm{z}$-direction is identical to that of a conventional wide-field microscope. Below, the terms resolution or resolving power are to be understood as referring exclusively to the x-direction.

The spatial confinement of fluorescence emission in the RESOLFT microscope of figure 2.12 , i.e. its resolving power, can be measured in a simple yet illustrative way by applying the illumination sequence depicted in figure 2.8A to a homogeneous film of the switchable marker and imaging the fluorescence light elicited by the probe pulse onto a CCD camera. Since the diffraction-limited detection causes a broadening of the fluorescence stripes when 


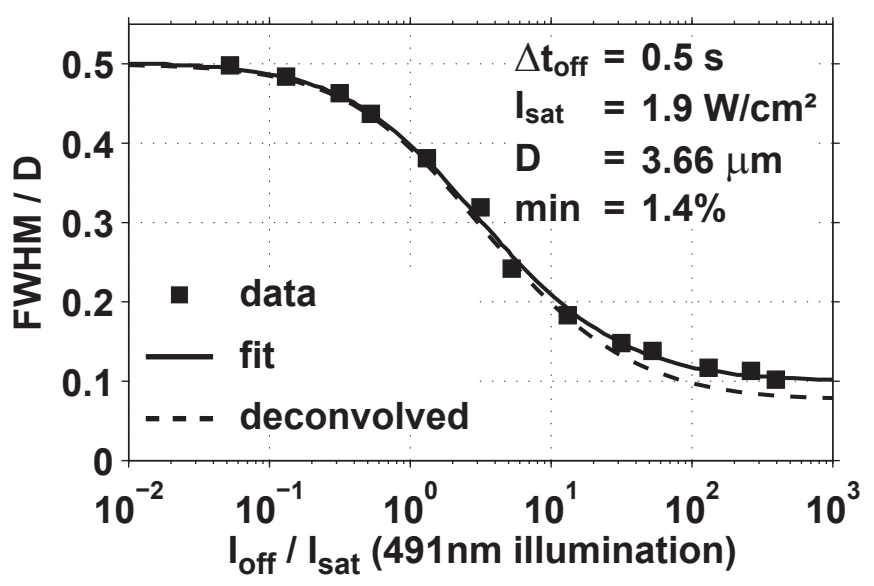

Figure 2.14: Analysis of the data parts of which are displayed in figure 2.13. FWHM/D vs $I_{\text {off }} / I_{\text {sat }}$, FWHM is a measure of the microscope's resolving power ( $D$ : stripe distance, $I_{\text {off }}$ : maximum of the depletion pattern). Solid line: fit taking into account stripe-broadening due to the diffraction-limited detection optics. Dashed line: true value of FWHM/D, i.e. after deconvolution with the detection PSF. - In principle, the resolving power of a RESOLFT microscope can be arbitrarily enhanced by increasing $I_{\text {off }} / I_{\text {sat }}$ (see $(1.10)$ ). In the measurements presented here non-perfect intensity zeros of the depletion light practically limited the attained resolution to $\approx D / 12$. In absolute numbers this corresponds to a resolution of $\approx 40-45 \mathrm{~nm}$ when choosing $D \approx \lambda \approx 500 \mathrm{~nm}$.

imaging them onto the $\mathrm{CCD}$ it is convenient to make $D$ as large as possible to keep the influence of this effect on the measured values to a minimum. For this reason a relatively large value for the distance $D$ between neighboring stripes was chosen in the experiment, namely $D=3.66 \mu \mathrm{m}$.

The result of this experiment as performed on purified rsFastLime embedded in a film of PVA is illustrated in figure 2.13. It depicts the fluorescence emission profile along the $\mathrm{x}$-direction for increasing switch-off intensities $I_{\text {off }}$. The curves displayed in figure $2.13 \mathrm{a}$ show the fluorescence signal normalized to the emission before switch-off; figure $2.13 \mathrm{~b}$ features the same data with each curve normalized to its maximum value. Probing of fluorescence in the last image acquisition step was performed with the same structured illumination pattern prior used for switch-off (see figure 2.8 $\mathbf{A}, 3 \mathrm{~b}$ ) ); in between switch-off and probing the pattern was shifted by half a period, i.e. $I_{\text {off }} \propto \sin ^{2}(\pi x / D)$ and $I_{\text {probe }} \propto \cos ^{2}(\pi x / D)$.

Clearly, increasing the switch-off intensity entails a gradual constriction of the fluorescence peaks, i.e. a higher resolving power of the microscope. This is most evident in figure $2.13 \mathrm{~b}$. Figure 2.13a additionally reveals that increasing the switch-off intensity leads to a concomitant decline of the peak signal. This unwanted effect can be attributed to non-perfect intensity zeros of the switch-off light.

Figure 2.14 illustrates the relation between resolving power and switch-off intensity in more detail. It shows an analysis of the experiment partially displayed in figure 2.13, featuring the full width at half maximum (FWHM) $\Delta x$ of the fluorescence stripes, i.e. the resolving power of the microscope, plotted against the switch-off intensity $I_{\text {off }}$ of the structured blue depletion light ( $I_{\text {off }}$ refers to the intensity maximum of the depletion pattern). It is 
practical to measure $\Delta x$ in multiples of the periodicity $D$ of the structured switch-off pattern as the dependence of $\Delta x / D$ on $I_{\text {off }}$ is invariant under a change of the absolute length scale (i.e. $\Delta x / D=$ const for a given value of $I_{\text {off }}$ ).

For $I_{\text {off }}=0, \Delta x$ is given by the illumination profile of the probe light, $I_{\text {probe }} \propto \cos ^{2}(\pi x / D)$. The $F W H M$ of this pattern is $D / 2$, hence $\Delta x / D=0.5$ for $I_{\text {off }}=0$. Increasing the switch-off intensity $I_{\text {off }}$ reduces the stripe width, in accordance with what can be observed in figure 2.13. The solid line shows a fit to the measured data. The dashed line corrects for the broadening of the fluorescence stripes due to the diffraction-limited detection optics and can be interpreted as representing the true resolving power of the microscope. A noticeable deviation between the two starts to set in where the absolute value of the stripe width approaches the size of the detection $\operatorname{PSF}\left(\Delta x_{P S F} \approx 230 \mathrm{~nm}\right)$.

Interestingly, the data displayed in figure 2.14 suggest that the resolving power of the microscope cannot be arbitrarily enhanced but approaches a value of $\Delta x / D \approx 1 / 12$ as the switch-off intensity is increased. Choosing $D \approx \lambda \approx 500 \mathrm{~nm}$ this value corresponds to an absolute resolution of $\approx 40-45 \mathrm{~nm}$. The reason for the limited resolution in the present case is the interplay between a non-zero intensity in the minima of the structured depletion light (due to e.g. optical imperfections or instabilities) and the photophysics of the switchable marker rsFastLime. A closer investigation shows that non-zero intensity minima of the switch-off light not only reduce the fluorescence signal as can be clearly observed in figure $2.13 \mathrm{a}$; in combination with the specific switch-off characteristic of rsFastLime they also limit the resolving power of the microscope.

This effect is illustrated in figure 2.15. It shows simulations featuring the dependence of the effective excitation profile $F(x)$ on the quality of the switch-off minima and on the applied switch-off intensity (see appendix A.1 for an analytic expression of $F(x)$ ). Remember that the peak width of $F(x)$ is a measure of the microscope's resolving power. For matters of completeness the case of both homogeneous and structured probing is studied, shown on the left and on the right, respectively. Figures $\mathbf{1 a}$ and $\mathbf{1 b}$ depict the intensity distributions of the switch-off- and the probe beam, with the minima of the intensity-modulated beam assumed to be non-zero. All intensities are normalized to one. The shape of the effective excitation profile for different values of the switch-off minimum can be seen in figures 2a and $\mathbf{2 b} . I_{\text {off }}$ is fixed at $50 \cdot I_{\text {sat }}$ for all curves. By contrast, figures $\mathbf{3 a}$ and $\mathbf{3 b}$ feature $F(x)$ for increasing switch-off intensity with a fixed minimum of $0.01 \cdot I_{\text {off }}^{\max }$. Clearly, the non-zero switch-off minimum gives rise to a decrease in the peak signal. Importantly, this unwanted effect results not only in a lower signal to noise ratio; in addition, it entails a broadening of the FWHM, i.e. a deterioration of the resolving power. This result is shown in figures $\mathbf{4 a}$ and $\mathbf{4 b}$ which depict a plot of the FWHM versus $I_{\text {off }}$ for increasing values of the switch-off minimum (compare this plot with figure 2.14). For non-perfect switch-off minima the attainable resolution is limited as evidenced by the fact that the value of FWHM/D remains finite as the switch-off intensity is increased. The dependence of the attainable resolution on the quality of the switch-off minima is approximately given by FWHM/D $\approx(2 / \pi) \sqrt{a}$, where $a$ is the switch-off minimum in units of the maximum switch-off intensity, i.e. $a:=I_{o f f}^{\min } / I_{\text {off }}^{\max }$ (see appendix A.1). 

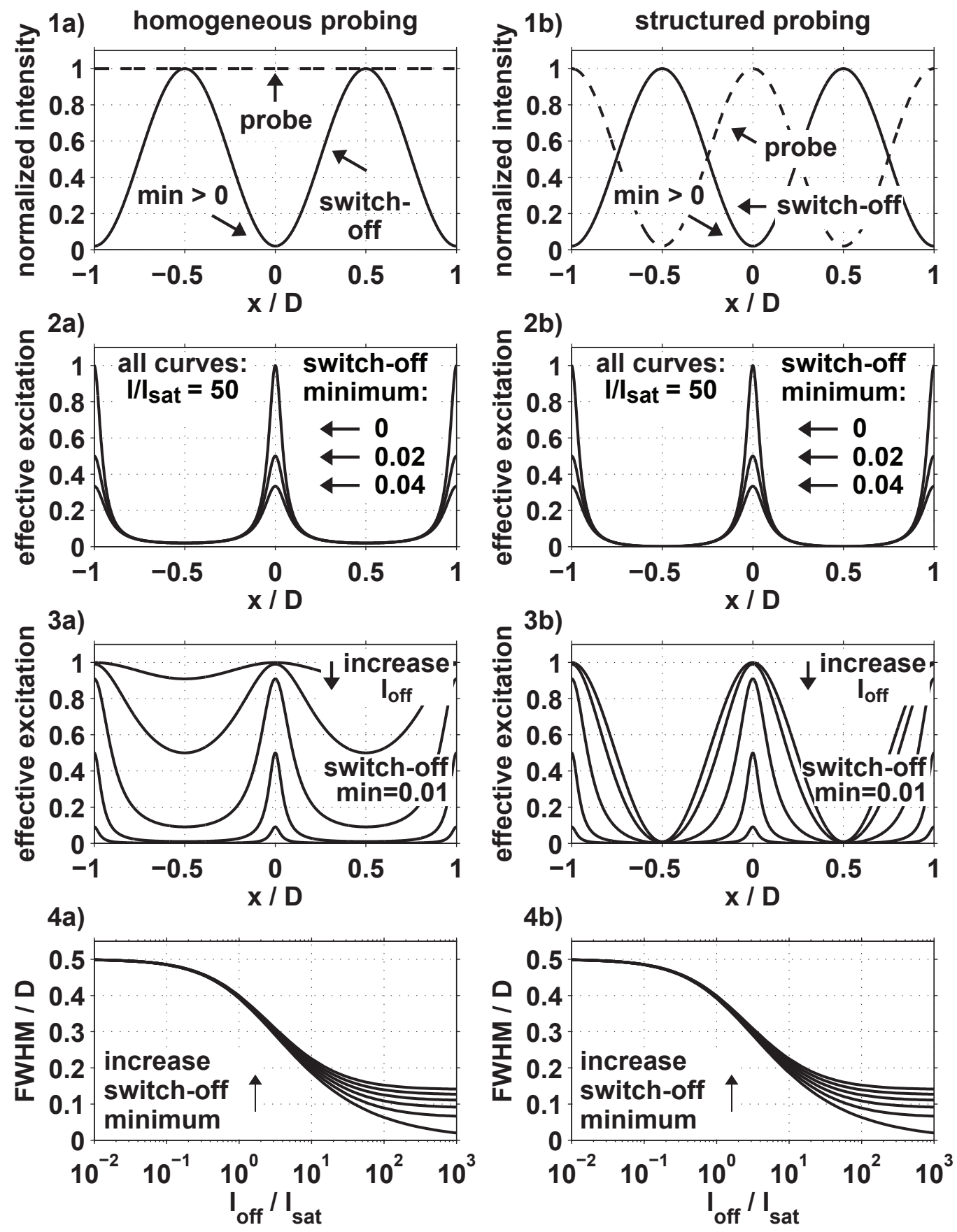

Figure 2.15: Effective excitation profile $F(x)$ (=emission probability) for homogeneous (left) and for structured probing (right) using the RSFP rsFastLime as a fluorescence marker. 1a+b Intensity distribution of the switch-off- and the probe beam, $I_{o f f}(x)$ and $I_{\text {probe }}(x)$. To illustrate the effect of optical imperfections on the resolving power, the intensity-modulated beam features a non-zero minimum $(\min >0)$. $\mathbf{2} \mathbf{a}+\mathbf{b} F(x)$ for three different values of the switch-off minimum, given in units of the maximum intensity. $\mathbf{3 a} \mathbf{a}+\mathbf{b} F(x)$ for increasing switch-off intensity $\left(I_{\text {off }}(x) / I_{\text {sat }}(x)=0.1,1,10,100,1000\right)$ and a switch-off minimum of $0.01 \cdot I_{o f f}^{\max } \cdot \mathbf{4 a} \mathbf{a}+\mathbf{b}$ FWHM of the effective excitation peaks (=resolving power) versus switch-off intensity for different values of the switch-off minimum $\left(I_{\text {off }}^{\min } / I_{\text {off }}^{\max }=0,0.01,0.02,0.03,0.04,0.05\right)$. As a consequence of the switching characteristic of rsFastLime a non-zero switch-off minimum limits the attainable resolving power. 
It is important to point out that wether or not the attainable resolving power is affected by the quality of the switch-off minima very much depends on the switching mechanism employed (cis-trans isomerization, triplet state saturation, stimulated emission) and on the photophysics of the marker molecule. The relevant quantity in this context is the shape of the depletion curve (see figures 2.10 and 2.11). If the latter is of the form $\eta\left(I_{\text {off }}\right)=1 /\left(1+I_{\text {off }} / I_{\text {sat }}\right)$ a nonzero switch-off minimum leads to a worsening of the resolving power as described above. Examples for this sort of depletion characteristic in RESOLFT microscopy are fluorescent protein switching [68, 74] and Ground State Depletion (GSD) [27, 28]. By contrast, if the depletion curve follows a single exponential (i.e. $\eta\left(I_{\text {off }}\right)=\exp \left(-I_{\text {off }} / I_{\text {sat }}\right)$ ) as i.e. in STED microscopy, a non-zero switch-off minimum naturally leads to a worsening of the signal to noise ratio; however, it does not entail a deterioration of the resolving power (see appendix A.1 ). In other words, the attainable resolution in a RESOLFT microscope based on the reversible switching of the RSFP rsFastLime is more sensitive to optical imperfections than it is in STED microscopy.

\section{Image Acquisition and -Reconstruction}

Image acquisition and -reconstruction in parallelized wide-field RESOLFT microscopy bear resemblance to the image formation process in confocal microscopy. In confocal imaging an excitation spot of known size is scanned over the sample in a controlled manner. The fluorescence light emitted at each spot position is focused on a point detector and the recorded signal assigned to the corresponding pixel in the final image. The resolving power of the microscope is determined by the size $\Delta x$ of the excitation spot and the stepsize $\Delta s$ used for sampling the specimen must be adjusted in such a way that $\Delta s \leq \Delta x / 2$ (Niquist criterion). Imaging with the parallelized wide-field RESOLFT microscope shown in figure 2.12 follows the same basic concept. However, instead of a single spot an excitation pattern $F_{\varphi}(x)$ featuring a periodic structure of stripe-like intensity peaks is scanned over the sample along the x-direction, the subscript $\varphi$ denoting the phase of the excitation pattern, i.e. its position relative to the sample. To enable simultaneous recording of the fluorescence signal elicited by all stripes a CCD camera replaces the point detector used in confocal microscopy. Owing to the periodicity of $F_{\varphi}(x)$ only one complete period of length $D$ needs to be scanned, which speeds up image acquisition considerably as compared to the confocal case.

Akin to confocal imaging the resolving power of the microscope along the $\mathrm{x}$-direction is given by the width $\Delta x$ of the excitation stripes while resolution along the $\mathrm{y}$ - and the $\mathrm{z}$ direction is identical to that of a conventional wide-field system. However, it should be noted that resolution beyond the diffraction limit can readily be extended to all directions by using a different switch-off profile. The enhanced x-resolution of the RESOLFT microscope shown in figure 2.12 over a confocal system or a conventional wide-field microscope stems from the fact that $\Delta x$ can be made arbitrarily small and is no longer subject to the diffraction limit (see (1.10) ). Notwithstanding, in the CCD image recorded at a particular pattern position the fluorescence signal elicited by the subdiffraction-sized excitation stripes is blurred by the diffraction-limited detection optics. However, since the exact position of the excitation profile is known the blurred CCD signal can be ascribed to the individual subdiffraction sized stripes of the excitation profile $F_{\varphi}(x)$. Importantly, for this assignment to be unam- 
biguous it is crucial that the distance $D$ between two neighboring stripes be at least twice the width of the diffraction-limited detection PSF, i.e. $D \geq \lambda / N A$. Reducing the stripe distance $D$ further would result in a CCD image in which fluorescence originating from neighboring stripes overlaps, rendering an unequivocal assignment impossible. Note again the analogy to image reconstruction in confocal microscopy where controlling the position of the excitation spot makes it possible to assign the recorded signal to a particular image pixel.

A simulation of the image acquisition and -reconstruction process in parallelized widefield RESOLFT microscopy is illustrated in figure 2.16 which exemplifies the procedure for two scanning steps, i.e. for two pattern positions with respect to the sample. The object consists of $50 \mathrm{~nm}$ beads assembled at varying distances (first row: $250 \mathrm{~nm}$, second row: $100 \mathrm{~nm}$ and $150 \mathrm{~nm}$, third row: $50 \mathrm{~nm}$ and $200 \mathrm{~nm}$ ). The total size of the individual images shown is $1.25 \mu \mathrm{m} \times 1.25 \mu \mathrm{m}$. Image number 1) features the subdiffraction excitation stripes $F_{\varphi}(x)$ at the top and the sample at the bottom (the excitation pattern extends over the whole field of view along the y-direction; for matters of clarity the stripes are not shown in full). The excitation maxima feature a FWHM of $\Delta x=32 \mathrm{~nm}$ as produced by a saturated sine squared depletion pattern and a saturation factor of $\xi:=I_{\text {off }} / I_{\text {sat }}=100$; the distance between neighboring stripes is $D=500 \mathrm{~nm}$. Image number 2) shows the emitted fluorescence as the beads are excited by the structured excitation profile, which is indicated by white arrows. The third image illustrates the fluorescence signal detected by the CCD camera. It is given by a convolution of the fluorescence emission distribution shown in image number 2) with the detection PSF. The latter is taken to be an airy function with FWHM $\Delta x_{P S F}=218 \mathrm{~nm}$ corresponding to an emission wavelength of $\lambda=500 \mathrm{~nm}$ and a numerical aperture of $\mathrm{NA}=1.4$. Again, the intensity peaks of the excitation profile are indicated by vertical white arrows whereas the minima are highlighted as white bars. Image number 4) illustrates the assignment of the fluorescence signal collected in between to neighboring minima of the excitation profile (white bars in 3) ) to the central maximum. This 'squeezed' signal constitutes the new 'pixels' which are added to the reconstructed super-resolution image in the fifth image. The pixel size in the reconstructed super-resolution image along the x-direction is given by the scanning stepsize $\Delta s$. For the simulation a value of $\Delta s=10 \mathrm{~nm}$ was chosen.

It shall be stressed at this point that for flawless image reconstruction it is absolutely essential that during data acquisition exactly one period of the excitation pattern be scanned, i.e. $D=n \cdot \Delta s(\Delta s$ : stepsize, $n$ : number of scanning steps). Not meeting this condition results in visible 'edges' in the final high-resolution image.

Figure 2.17 shows the bead object, the conventional wide-field image, i.e. the convolution of the object with the detection PSF, and the RESOLFT recording with x-confinement of fluorescence. Evidently, the latter features enhanced resolution along the x-direction: objects which cannot be discerned in the conventional wide-field view appear clearly separated in the RESOLFT image. Along the y-direction on the other hand both the conventional as well as the RESOLFT image exhibit the same diffraction-limited resolution. Extending resolution enhancement to 2D or 3D can easily be achieved by using a switch-off pattern which provides confinement of fluorescence along all dimensions. 

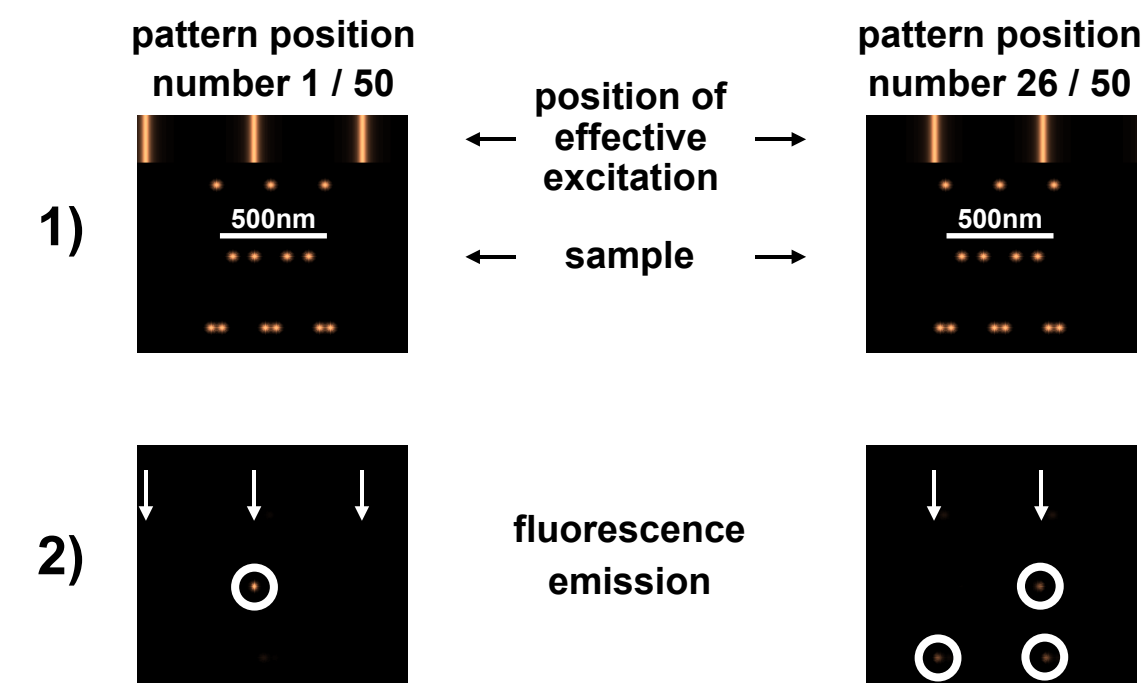

\section{fluorescence emission}
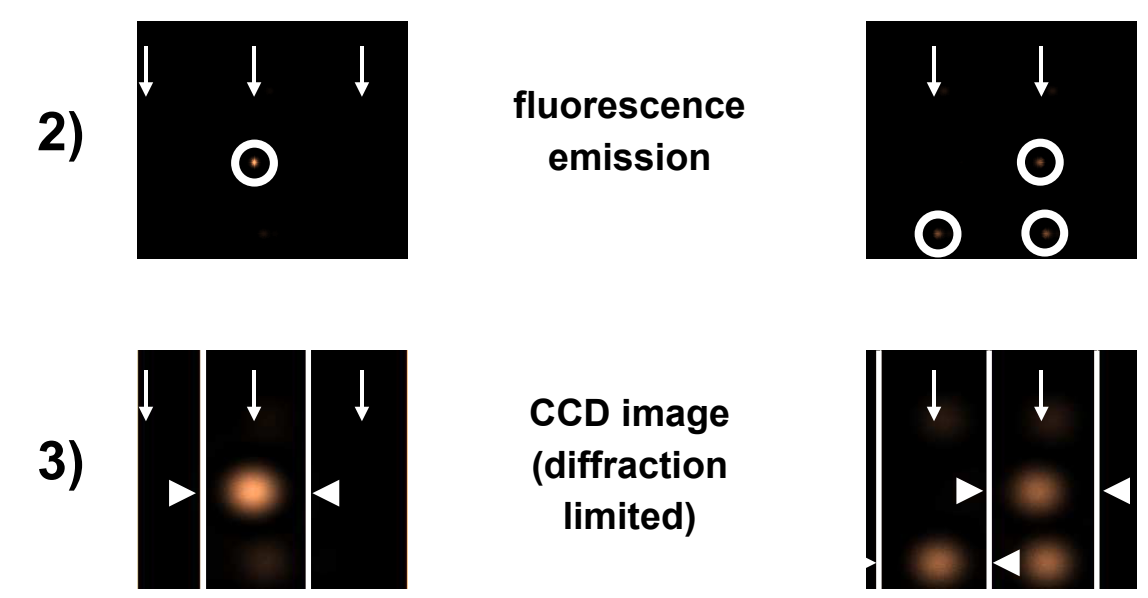

\section{CCD image (diffraction limited)}
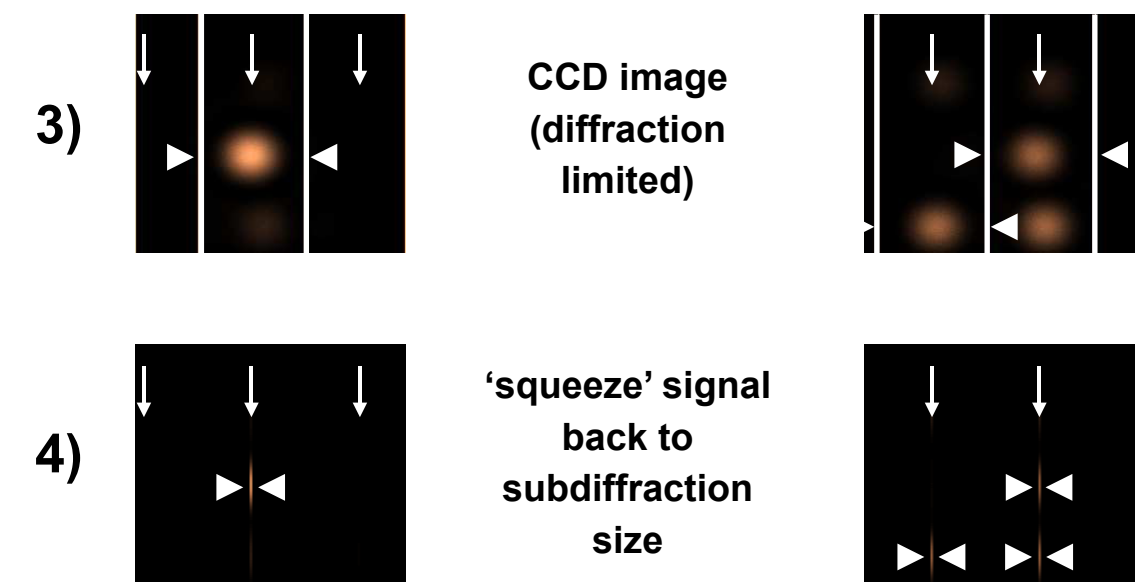

$$
\begin{gathered}
\text { 'squeeze' signal } \\
\text { back to } \\
\text { subdiffraction } \\
\text { size }
\end{gathered}
$$

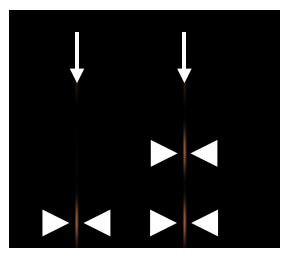

$$
\text { add to }
$$
reconstructed subdiffraction image

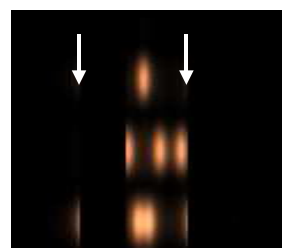

Figure 2.16: Simulation of the imaging- and the image reconstruction process in parallelized wide-field RESOLFT microscopy providing subdiffraction resolution along the $\mathrm{x}$-direction. The process is exemplified for two positions of the subdiffraction-sized effective excitation profile relative to the sample (indicated by vertical white arrows in images number 2) to 5) ). To record a complete image with super-resolution along the $\mathrm{x}$-direction the pattern must be scanned over the sample for one complete period. 1) Effective excitation profile after switch-off (top) and sample (bottom). 2) Fluorescence emitted from the sample. 3) diffraction-limited CCD image of the emitted fluorescence. 4) Assignment of the detected signal to the maxima of the effective excitation profile. 5) Adding of the new pixels to the reconstructed super-resolution image. 


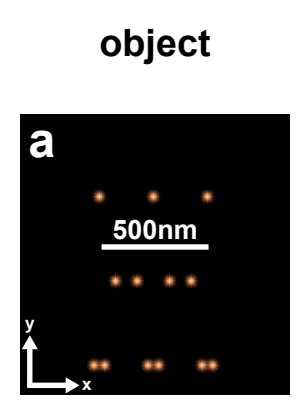

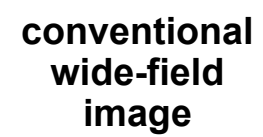

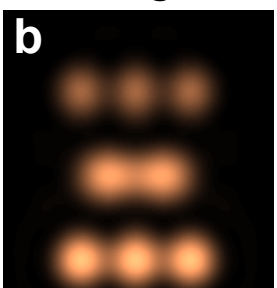

RESOLFT

image

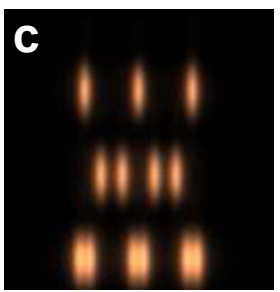

Figure 2.17: Simulation of an object $\mathbf{a}$ along with a conventional wide-field image $\mathbf{b}$ and a RESOLFT recording $\mathbf{c}$ of the same object. The RESOLFT image features enhanced resolution along the $\mathrm{x}$-direction. Along $\mathrm{y}$, the resolving power in $\mathbf{b}$ and $\mathbf{c}$ is identical. For the simulation of the RESOLFT image perfect switch-off minima and an intensity $I_{o f f}=100 \cdot I_{\text {sat }}$ were assumed. With these parameters the resolution along the x-direction is $32 \mathrm{~nm}$.

When using a fluorescence marker which cannot be fully switched to the dark state the reconstruction process described above needs to be complemented by one additional step if the complete subdiffraction information is to be extracted. E.g., non-complete switchoff may be caused by switch-on cross talk of the excitation light as in rsFastLime. It can be described by a constant offset term in the depletion curve. In the case of rsFastLime the latter is of the form $\eta\left(I_{\text {off }}\right)=r+(1-r) /\left(1+I_{\text {off }} / I_{\text {sat }}\right)$ (see (2.3)). The constant offset $r$ gives rise to an overlay of the super-resolution information with a diffraction-limited component which would impair resolution enhancement if it was not corrected for. In the following it shall be demonstrated how this problem can be remedied.

The wide-field CCD image $G_{\varphi}(x)$ taken at one particular position (i.e. phase value $\varphi$ ) of the switch-off pattern is given by

$$
G_{\varphi}(x)=\left(\rho(x) \cdot F_{\varphi}(x)\right) \otimes h(x)
$$

where $\rho(x), F_{\varphi}(x)$ and $h(x)$ are, respectively, the dye distribution, the effective excitation and the diffraction-limited wide-field detection PSF; ' $\otimes$ ' denotes the convolution operator. $F_{\varphi}(x)$, on the other hand, can be written as

$$
\begin{aligned}
F_{\varphi}(x) & =I_{\text {probe }}(x) \cdot \eta\left(I_{\varphi}(x)\right) \\
& =I_{\text {probe }}(x) \cdot\left(r+\frac{1-r}{1+I_{\varphi}(x) / I_{\text {sat }}}\right) \\
& =r \cdot \underbrace{I_{\text {probe }}(x)}_{\text {conventional }}+(1-r) \cdot \underbrace{\frac{I_{\text {probe }}(x)}{1+I_{\varphi}(x) / I_{\text {sat }}}}_{\text {RESOLFT }} .
\end{aligned}
$$

I.e. $F_{\varphi}(x)$ can be decomposed into a 'conventional' and a 'RESOLFT' component of which only the latter carries super-resolution spatial frequencies. The recorded CCD image $G_{\varphi}(x)$ 
can be decomposed accordingly:

$$
G_{\varphi}(x)=r \cdot \underbrace{G_{\varphi}^{C}(x)}_{\text {conventional }}+(1-r) \cdot \underbrace{G_{\varphi}^{R}(x)}_{\text {RESOLFT }} .
$$

In other words, the super-resolution information can be extracted from the the CCD image $G_{\varphi}(x)$ by recording a reference image $G_{\varphi}^{C}(x)$ without application of the switch-off light, scaling it down by a factor of $r$ and subsequently subtracting it from $G_{\varphi}(x)$.

\section{Fourier Space Reconstruction}

Very briefly, an alternative approach to image reconstruction in super-resolution wide-field microscopy shall be discussed. It relies on the use of structured illumination in combination with data processing in the Fourier domain. The essence of this very interesting concept is that by scanning a periodic, spatially modulated illumination profile over the object and by taking images at each scanning step, spatial information about the sample structure can be gained which lies beyond the resolving power of the diffraction limited detection optics of the imaging apparatus. The first proposal to increase the optical resolution using structured illumination was put forward by Lukosz and Marchand [75]. A first practical implementation of this concept was published by Heintzmann and Cremer [76]. Later, Gustafsson [77] and Frohn [78] used structured illuminatin to surpass the diffraction limit by a factor of two. Inspired by advances in super-resolution point-scanning microscopy [13, 19], Heintzmann et al. extended the concept of resolution enhancement by non-linear phenomena to widefield microscopy [79]; the first experimental realization was put forward by Gustafsson, who demonstrated a lateral resolution of 50nm [80]. Recently, structured illumination microscopy with resolution enhancement by a factor of two was extended to the 3D imaging of biological samples [81, 82, 83]. A recent review by Stemmer et al. [84] gives a summary of related wide-field techniques.

Here, only the basic idea underlying resolution enhancement in optical microscopy via structured illumination shall be presented. In particular, only the one-dimensional case shall be discussed. It allows to point out the main features of the concept while keeping the description simple. A comprehensive account of the theory and the experimental realization of the methodology can be found in [82].

The properties of an optical system are completely characterized by its point spread function (PSF) $h(x)$, which describes the image of a point like object. The image $B(x)$ of an extended object $\rho(x)$ whose emission is given by an emission probability $J(x)$ can be written as

$$
B(x)=(\rho(x) \cdot J(x)) \otimes h(x),
$$

where ' $\otimes$ ' may denote the convolution operator. The convolution with a PSF $h(x)$ of finite extent results in an effective blurring of the object in the image plane. Thus, the width of $h(x)$ determines the resolving power of the optical system.

By virtue of the convolution theorem the Fourier transform of (2.8) is given by

$$
\tilde{B}(k)=(\tilde{\rho}(k) \otimes \tilde{J}(k)) \cdot \tilde{h}(k),
$$


where a tilde $(\sim)$ denotes the Fourier transform of the corresponding real space quantity. $\tilde{h}(k)$, the Fourier transform of the PSF $h(x)$, is called the optical transfer function (OTF). It describes how strongly and with what phase shift the spatial frequency $k$ of the object is transferred into the measured data. In Fourier space, the limited resolution of the optical system manifests itself in the fact that the OTF $\tilde{h}(k)$ has a finite region of support, outside of which it is zero. I.e., large spatial frequencies, which correspond to small structures in real space, are not transferred by the imaging system. Clearly, increasing the resolving power of the system is equivalent to transmitting information from outside of the region of support of the OTF.

If the emission probability $J(x)$ features a periodic structure with periodicity $D$ it may be written as a Fourier series:

$$
J_{\varphi}(x)=\sum_{n=-\infty}^{\infty} c_{n} e^{i n(p x+\varphi)},
$$

where $p=2 \pi / D$. Importantly, the emission probability $J_{\varphi}(x)$ may include higher harmonics of arbitrary order if a non-linear relation exists between the emitted light and the applied illumination. In the case of fluorescence microscopy, examples for such a non-linear relation include saturation of the excited state [80] or protein photoswitching as demonstrated in [68] or in this thesis. The phase $\varphi$ has been included in the expression for the emission probability to point out that the latter can be scanned over the object. The value of the phase $\varphi$ then determines the position of $J_{\varphi}(x)$ with respect to the sample.

The Fourier transform of 2.10) reads

$$
\tilde{J}_{\varphi}(k)=\sum_{n=-\infty}^{\infty} c_{n} \delta(k-n p) e^{i n \varphi}
$$

After inserting this expression for $\tilde{J}_{\varphi}(k)$ in equation (2.9) and carrying out the convolution, the image Fourier transform $\tilde{B}_{\varphi}(k)$ takes on the following form

$$
\tilde{B}_{\varphi}(k)=\left(\sum_{n=-\infty}^{\infty} c_{n} \tilde{\rho}(k-n p) e^{i n \varphi}\right) \cdot \tilde{h}(k) .
$$

This means that the image Fourier transform $\tilde{B}_{\varphi}(k)$ is essentially a sum of identical copies of the object Fourier transform $\tilde{\rho}(k)$ which are shifted laterally in reciprocal space by a distance $n p=2 \pi n / D$. This, in turn, implies that previously inaccessible information is shifted inside the region of support of the OTF. Exact knowledge of both the OTF $\tilde{h}(k)$ and the Fourier transform of the emission probability $\tilde{J}_{\varphi}(k)$ now allows to extract the shifted information components $\tilde{\rho}(k-n p)$. A number of $2 N+1$ of these components can be determined by taking $2 N+1$ images with different phase values $\varphi_{i}(i=-N, \ldots, N)$ and solving the following system of $N+1$ linear equations

$$
\tilde{B}_{\varphi_{i}}(k)=\tilde{h}(k) \cdot \sum_{n=-N}^{N} c_{n} \tilde{\rho}(k-n p) e^{i n \varphi_{i}}, \quad i=-N, \ldots, N
$$


with the $2 N+1$ unknowns $\tilde{\rho}(k-n p) \quad(n=-N, \ldots, N)$ for each value of $k$ inside the region of support of the OTF. Since the Fourier transform of a real valued function such as $\rho(x)$ obeys the symmetry property $\tilde{\rho}(-k)=(\tilde{\rho}(k))^{*}$, where ${ }^{*}$ ', denotes complex conjugation, (2.13) has to be solved for either positive or negative $k$-values only. Once the shifted components $\tilde{\rho}(k-$ $n p$ ) have been identified they can be re-shifted to their original position in reciprocal space, effectively extending the region of support of the OTF. Re-transformation of the enlarged object Fourier transform $\tilde{\rho}(k)$ readily yields a super-resolution image.

In practice, image reconstruction by Fourier space analysis as described in this section is difficult to realize as the method assumes the exact knowledge of the illumination pattern, the phase value $\varphi$ and, if applicable, the non-linear relation between fluorescence emission and applied illumination (as e.g. in $S_{1}$-saturation or protein photoswitching). However, incomplete knowledge of any of the aforementioned parameters may easily result in artifacts in the reconstructed super-resolution image. It is for these reasons that in this work the 'direct' image reconstruction approach as outlined in figure 2.16 was persued.

\section{Imaging of Biological Samples}

In the following it shall be demonstrated that parallelized wide-field RESOLFT microscopy based on the reversible switching of the RSFP rsFastLime can be used to image biological specimens at subdiffraction resolution. Here, this assertion is validated for super-resolution imaging along one lateral dimension. To this end the endoplasmatic reticulum (ER) of PtK2 cells (kidney cells from the rat kangaroo Potorous tridactylus) was volume-stained with rsFastLime via transfection with a construct carrying an ER targeting- and retention signaling sequence. The ER is an organelle which mainly serves the folding and distribution of membrane proteins. It consists of a network of tubules and vesicles and can be found in all eucaryotic cells. For RESOLFT imaging cells were grown on a cover slip and fixated with Paraformaldehyde (PFA).

The image acquisition protocol followed the scheme outlined in figure $2.8 \mathbf{A}, 3 \mathbf{b}$. The sine squared blue $(\lambda=491 \mathrm{~nm})$ switch-off pattern with a period length of $D=460 \mathrm{~nm}$ was scanned over the sample for one period. Scanning was performed in 15 steps, corresponding to a step size of $\Delta s=30 \mathrm{~nm}$. In each step the sample was initially activated with a pulse of $403 \mathrm{~nm}$ light $\left(I_{403}=25 \mathrm{~W} / \mathrm{cm}^{2}, \Delta t_{403}=0.1 \mathrm{~s}\right)$. Following activation, the structured switch-off beam was applied. The switch-off time was $\Delta t_{o f f}=0.1 \mathrm{~s}$ and the peak intensity was chosen to be $I_{\text {off }}=400 \mathrm{~W} / \mathrm{cm}^{2}$, corresponding to a value of $I_{\text {off }} / I_{\text {sat }} \approx 50$. The pattern was then shifted by half a period and a probe beam with a peak intensity of $I_{\text {probe }}=10 \mathrm{Wcm}^{2}$ applied for $\Delta t_{\text {probe }}=0.02 \mathrm{~s}$. Concurrently, the CCD camera was exposed for $\Delta t_{C C D}=\Delta t_{\text {probe }}=0.02 \mathrm{~s}$. With these parameters a resolution of $\approx 42 \mathrm{~nm}$ could be expected, provided the minima of the switch-off beam featured zero intensity (see appendix A.1 for an expression of the resolving power). With the more realistic minimum value of $\approx 0.02$ (measured in units of the peak intensity) the anticipated resolution was $\approx 58 \mathrm{~nm}$. Before the recording of the RESOLFT image a conventional wide-field image was taken with homogeneous illumination $\left(I_{\text {probe }}=10 \mathrm{Wcm}^{2}, \Delta t_{C C D}=\Delta t_{\text {probe }}=0.02 \mathrm{~s}\right)$. 

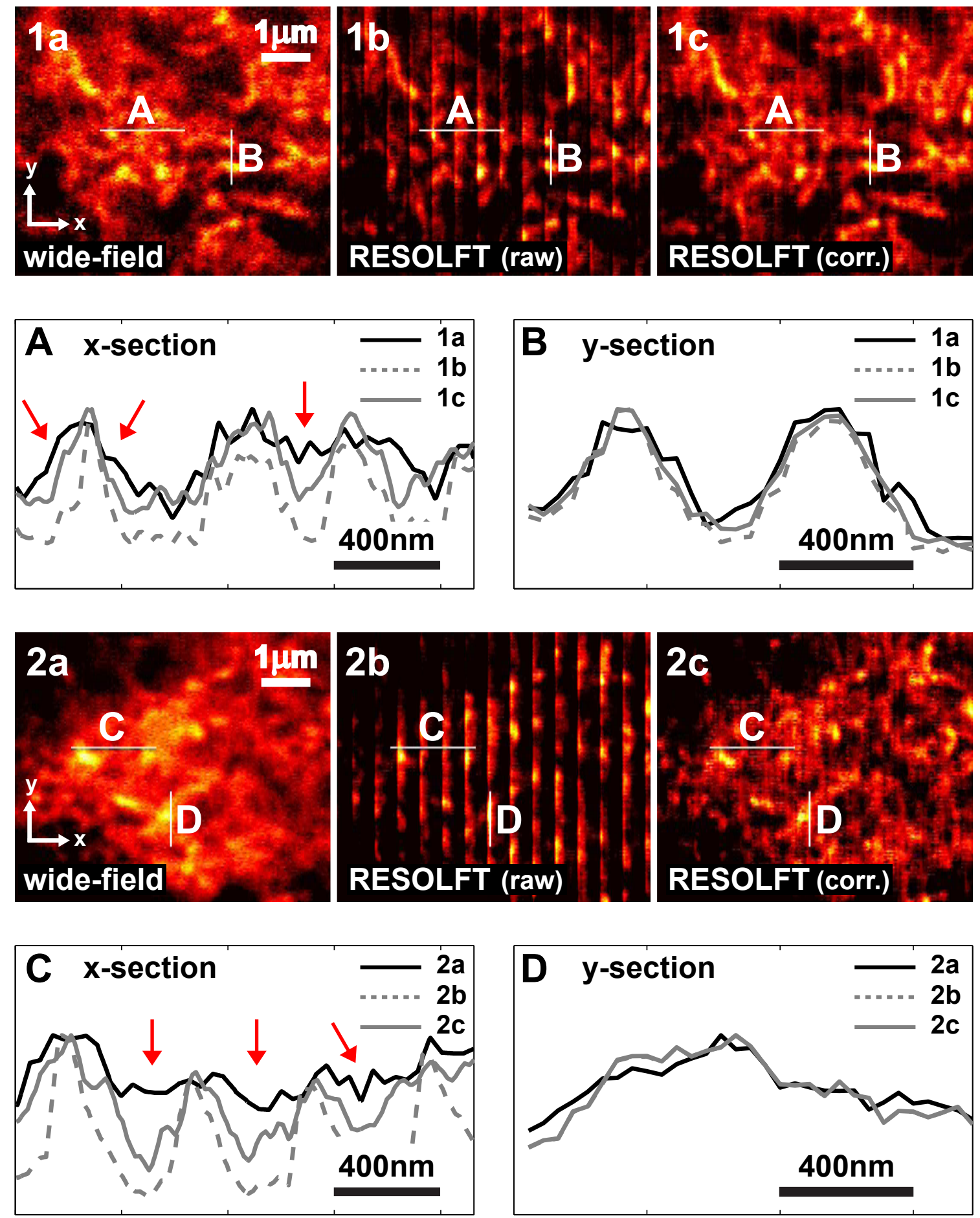

Figure 2.18: Comparison of wide-field- and RESOLFT images of the endoplasmatic reticulum (ER) of fixed PtK2 cells stained with the RSFP rsFastLime. The RESOLFT raw data reveal artifacts caused by irreversible photobleaching $(\mathbf{1 b}, \mathbf{2 b})$. In the final RESOLFT images $(\mathbf{1 c}, \mathbf{2} \mathbf{c})$ these artifacts have been corrected by rescaling the brightness of the images taken at different steps during acquisition. As compared to the conventional widefield recordings the final RESOLFT images reveal enhanced resolution along the $\mathrm{x}$-direction (see red arrows in the $\mathrm{x}$-sections $\mathbf{A}$ and $\mathbf{C}$ ). By contrast, no resolution enhancement is observed along the y-direction, as expected (see y-sections $\mathbf{B}$ and $\mathbf{D})$. 
Two examples of these recordings are shown in figure 2.18 (1a-c and 2a-c), each fea-

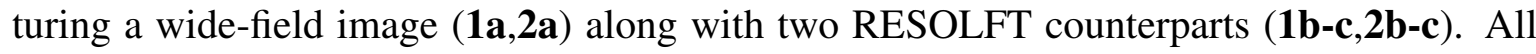
pictures are normalized to their respective maximum value. Also, in order to suppress background and increase image contrast the colormap minimum in each image is set to $20 \%$ of the maximum value. The pictures in the center $(\mathbf{1 b}, \mathbf{2 b})$ represent the RESOLFT data after image reconstruction following the algorithm described in figure 2.17. Clearly, they exhibit a sawtooth-like brightness profile along the $\mathrm{x}$ direction which can be attributed to irreversible photobleaching occurring in the course of the image acquisition process. The effect is particularly pronounced at the 'edges' where neighboring pixels contain the signals recorded during the first and the last image acquisition step. As a result the periodicity $D$ of the switch-off pattern is distinctly visible in the reconstructed image. The RESOLFT images on the right $(\mathbf{1 c}, \mathbf{2 c})$ represent an attempt to correct for these photobleaching artifacts: before image reconstruction the over all brightness of each of the 15 images recorded during different scanning steps was rescaled in such a way that the total count rate matched that of the first phase image. The two inevitable drawbacks of this sort of rescaling procedure are an unfavorable amplification of background noise and the fact that local inhomogeneities in brightness and bleaching behavior are not accounted for. In the corrected RESOLFT images $(\mathbf{1 c}, 2 \mathbf{c})$ the artifacts are significantly reduced. Similar artifacts may occur in the reconstructed view if the scanning distance $n \cdot \Delta s$ does not match the period length $D$.

The bleaching-corrected RESOLFT images (1c,2c) exhibit resolution enhancement along the $\mathrm{x}$-direction as exemplified by the line profiles $\mathbf{A}$ and $\mathbf{C}$. All line profiles are normalized to their respective maximum value. The red arrows point out the features which can be separated in the corrected RESOLFT image (grey line) but are not distinguishable in the conventional wide-field view (black line). The dashed grey line represents the corresponding profile of the RESOLFT raw data. As expected, no increase in the resolving power can be observed along the y-direction. This is evidenced by the line profiles $\mathbf{B}$ and $\mathbf{D}$ which yield virtually identical results

\subsection{Detailed Ensemble Photophysics of rsFastLime}

A number of experimental observations concerning the photophysics of rsFastLime cannot be satisfactorily described by the simple two-state model shown in figure 2.7. These include the seemingly non-complete activation with ultra violet light for short pulse durations and high intensities (see figure 2.9), the slight dependence of the achievable level of fluorescence depletion on the duration of the switch-off pulse (see figure 2.10), the relation between saturation intensity and switch-off duration as given in (2.4) and the photobleaching observed during RESOLFT image acquisition (see figure 2.18). Also, single molecule studies of the closely related RSFP Dronpa [38] gave proof of the existence of at least one additional metastable dark state in that protein [70, 85] (apart from the triplet state with a lifetime of $\approx \mu \mathrm{s}$ ), making it seem likely that a similar behavior could be found in rsFastLime.

To gain a more detailed understanding of the photophysical processes relevant to the use of rsFastLime in RESOLFT microscopy further experiments were carried out. These measurements gave clear evidence of the presence of a second short lived dark state of the rsFastLime 

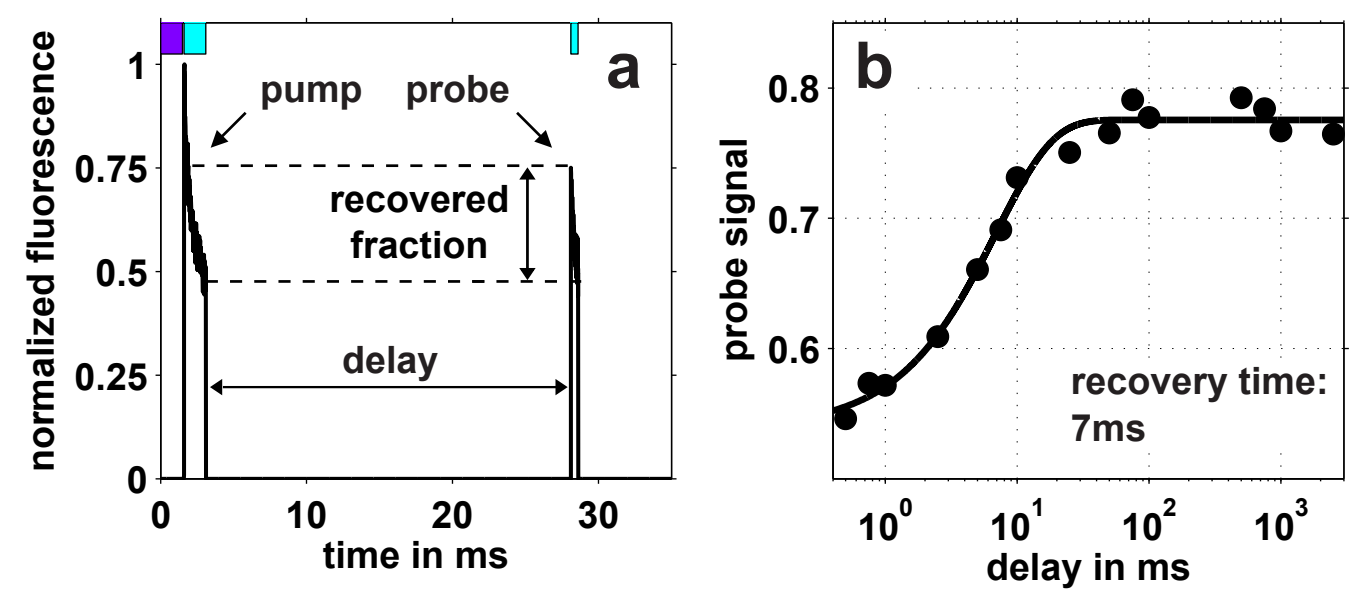

Figure 2.19: Evidence for the existence of a second metastable dark state (named D2 in the following) of the RSFP rsFastLime. The measurement was performed on an ensemble of the purified protein embedded in a film of PVA. a After switching off $\approx 50 \%$ of protein molecules a fraction of them spontaneously recover to the fluorescent state as verified by a second probe pulse. b Probe signal plotted versus the delay between pumpand probe pulse. The black curve represents a single exponential fit to the data suggesting that spontaneous recovery occurs from a single short lived dark state. The recovery time is $7 \mathrm{~ms}$. Experimental parameters: $I_{\text {off }}=I_{\text {probe }}=1 \mathrm{~kW} / \mathrm{cm}^{2}, \Delta t_{\text {pump }}=1.5 \mathrm{~ms}, \Delta t_{\text {probe }}=0.5 \mathrm{~ms}$.

chromophore, reminiscent of what had previously been found in the case of Dronpa.

The signature of this additional dark state is clearly exposed in the experiment depicted in figure 2.19. An ensemble of purified rsFastLime molecules embedded in PVA was illuminated with $491 \mathrm{~nm}$ light at an intensity of $I_{\text {off }}=1 \mathrm{~kW} / \mathrm{cm}^{2}$ for a duration of $\Delta t_{\text {off }}=1.5 \mathrm{~ms}$; after this switch-off pulse fluorescence was depleted to approximately $50 \%$ of its initial value. Probing the bright state population at later instances with another $491 \mathrm{~nm}$ pulse of the same intensity revealed that it partially recovered within $\approx 10 \mathrm{~ms}$. Fluorescence could be restored to its initial value upon illumination with an additional pulse of UV activation light following the probe pulse (not shown). Figure $2.19 \mathrm{~b}$ features the probe signal plotted versus the delay time along with a mono-exponential fit. Together, these findings point at the existence of a dark state with a lifetime of $\approx 7 \mathrm{~ms}$. Note that this state is clearly different from the stable dark state considered thus far. In the following, the two dark states shall be referred to as $D 1$ (stable, UV activatable) and D2 (short lived).

Populating the short lived dark state $D 2$ is clearly unfavorable for RESOLFT imaging. Relaxation of molecules from $D 2$ back to the fluorescent state during application of the probe pulse leads to an effectively less efficient depletion of the bright state and hence to a decrease in resolution. To investigate these effects a more in-depth analysis of the molecular state dynamics of rsFastLime was undertaken. A measurement which allows to determine the population of the fluorescent singlet state $S$, the dark states $D 1$ and $D 2$ as well as the fraction $B$ of irreversibly bleached molecules is outlined in figure 2.20 . A first $403 \mathrm{~nm}$ activation pulse $\left(\Delta t_{403}=0.1 \mathrm{~s}, I_{403}=50 \mathrm{~W} / \mathrm{cm}^{2}\right)$ transferred rsFastLime molecules to the fluorescent state $S$. The number of emitters in $S$ was then tested by a weak $491 \mathrm{~nm}$ probe pulse 


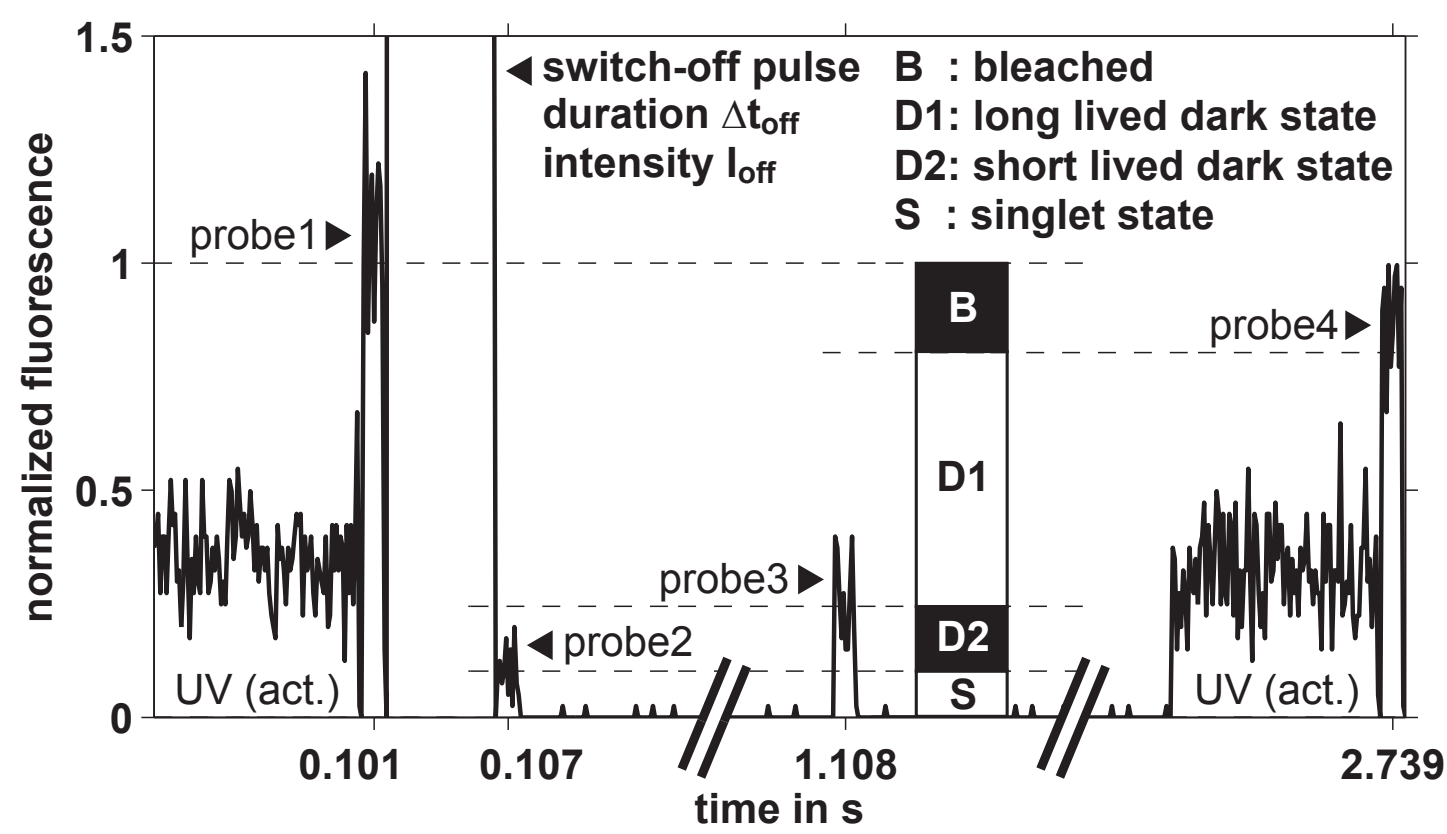

Figure 2.20: Ensemble of purified rsFastLime in polyvinyl alcohol (PVA): measuring the dependence of the fraction of molecules in state $S$ (fluorescent state), $D 1$ (stable dark state), $D 2$ (short lived dark state) and $B$ (bleached state) on the duration $\Delta t_{\text {off }}$ and the intensity $I_{\text {off }}$ of the applied $491 \mathrm{~nm}$ switch-off pulse. The relative population of the individual molecular states can be inferred from the signals generated by the $491 \mathrm{~nm}$ probe pulses 1-4. Probe1: fraction of molecules initially in the fluorescent state $S$ (normalized to one). Probe2: fraction of molecules left in $S$ immediately after switch-off. Probe3: fraction of molecules in $S$ after complete spontaneous recovery from the short lived dark state D2. Probe4: fraction of molecules in $S$ after the next activation pulse. The fractions of molecules found in the various molecular states are represented by the bar chart in the center right of the figure (see labels $B, D 1, D 2, S$ ). Parameters for the measurement on display: UV-activation: $I_{403}=50 \mathrm{~W} / \mathrm{cm}^{2}, \Delta t_{403}=100 \mathrm{~ms}$ (only the final $10 \mathrm{~ms}$ are shown). Probe pulses: $I_{\text {probe }}=$ $10 \mathrm{~W} / \mathrm{cm}^{2}, \Delta t_{\text {probe }}=1 \mathrm{~ms}$. Switch-off pulse: $I_{\text {off }}=10 \mathrm{~kW} / \mathrm{cm}^{2}, \Delta t_{\text {off }}=5 \mathrm{~ms}$.

(probe1; parameters for all probe pulses: $\Delta t_{\text {probe }}=1 \mathrm{~ms}, I_{\text {probe }}=10 \mathrm{~W} / \mathrm{cm}^{2}$ ), followed by another $491 \mathrm{~nm}$ switch-off pulse of varying duration and intensity. The fraction of molecules remaining in the fluorescent state after switch-off was inferred with a second probe pulse (probe2). Following a one-second delay a third probe pulse (probe3) tested the bright state population after complete recovery of emitters from the short lived dark state D2 (lifetime $\approx 7 \mathrm{~ms}$ ). Finally, markers in the stable dark state $D 1$ were transferred back to the fluorescent state $S$ via application of another $403 \mathrm{~nm}$ activation pulse and the bright state population after switching-on was checked by a fourth probe pulse (probe4). It must be mentioned that possible photobleaching caused by the ultra violet switch-on light was neglected in this experiment which may lead to a slight overestimation of the photobleaching generated by the switch-off beam. However, the results shown below indicate that this effect was of minor significance. Figure 2.20 illustrates how the population of the various molecular states can be determined from the fluorescence signals elicited by the probe pulses 1-4.

Figure 2.21 illustrates the dependence of the relative population of the molecular states 
$S$ (fluorescent, blue), $D 1$ (stable dark state, red), $D 2$ (short lived dark state, green) and $B$ (bleached fraction, black) on the $491 \mathrm{~nm}$ switch-off intensity, with the three plots a-c corresponding to different values of the switch-off time $\Delta t_{\text {off }}$. Intensities are given in units of the saturation intensity $I_{\text {sat }}$ which constitutes the relevant resolution measure in RESOLFT microscopy. To fit the data a photophysical four-state model of rsFastLime was used; it is outlined in some more detail further below.

The results displayed in figure 2.21 contain valuable information regarding the use of rsFastLime as a switchable marker in RESOLFT microscopy. Ideally, the switch-off intensity $I_{o f f}$ and the duration of the switch-off pulse $\Delta t_{\text {off }}$ should be chosen in such a way that molecules are transferred from the fluorescent state $S$ (blue) to the stable dark state $D 1$ (red); by contrast, populating the bleached state $B$ (black) as well as the short lived dark state D2 (green) should be avoided. The measured data indicate that these conditions are best met when long switch-off times and low intensities are employed, such as in c. This can best be seen when comparing the population of the various states in graphs a-c at a fixed value of the scaled intensity $I_{\text {off }} / I_{\text {sat }}$ (note that because of the different values of the saturation intensities $I_{\text {sat }}$ the absolute values of $I_{\text {off }}$ vary by a factor of $\approx 9$ between graphs). At $I_{o f f} / I_{\text {sat }}=100$ e.g. photoswitching from $S$ to $D 1$ is not efficient in a as a high percentage of emitters are either irreversibly bleached (state $B$, black) or shifted to $D 2$ (green). In b and $\mathbf{c}$ on the other hand occupation numbers are shifted more towards $D 1$ (red) while at the same time irreversible photobleaching (black) and the population of $D 2$ are reduced. Also, the fluorescent state (blue) is depleted more effectively. In other words, the photoswitching properties of rsFastLime can be improved at the expense of longer image acquisition times which in turn put higher demands on the stability of the sample and on the stability of the intensity minima of the structured switch-off pattern. In addition, the data reveal that irreversible photobleaching of rsFastLime occurs predominantly via the stable dark state $D 1$. This important insight highlights that the photophysics of this RSFP imposes limitations on its use as a switchable marker in RESOLFT microscopy: populating the stable dark state $D 1$ cannot be circumvented since it constitutes an integral part of the super-resolution imaging process.

The time evolution of the occupation numbers of the molecular states $S, D 1, D 2$ and $B$ is depicted in figure 2.22, the graphs a-c corresponding to the time evolution recorded for different values of the $491 \mathrm{~nm}$ switch-off intensity $I_{\text {off }}$. To facilitate comparison of the occupation numbers displayed in a-c the switch-off time $\Delta t_{\text {off }}$ is given in units of $\Delta t_{1 / 2}$, the time needed to deplete the fluorescent state $S$ (blue) by $50 \%$. The data underscore again that photoswitching of rsFastLime in RESOLFT microscopy is best carried out at low light intensities as shown in figure $2.22 \mathrm{a}$. Employing higher intensities entails increased photobleaching (black) and an elevated occupation of the short lived dark state D2 (green). Future research aimed at improving the photoswitching characteristics of rsFastLime with respect to its use in RESOLFT imaging should therefore try to find a chromophore which features reduced photobleaching rates even at higher switch-off intensities to enable fast image acquisition. 

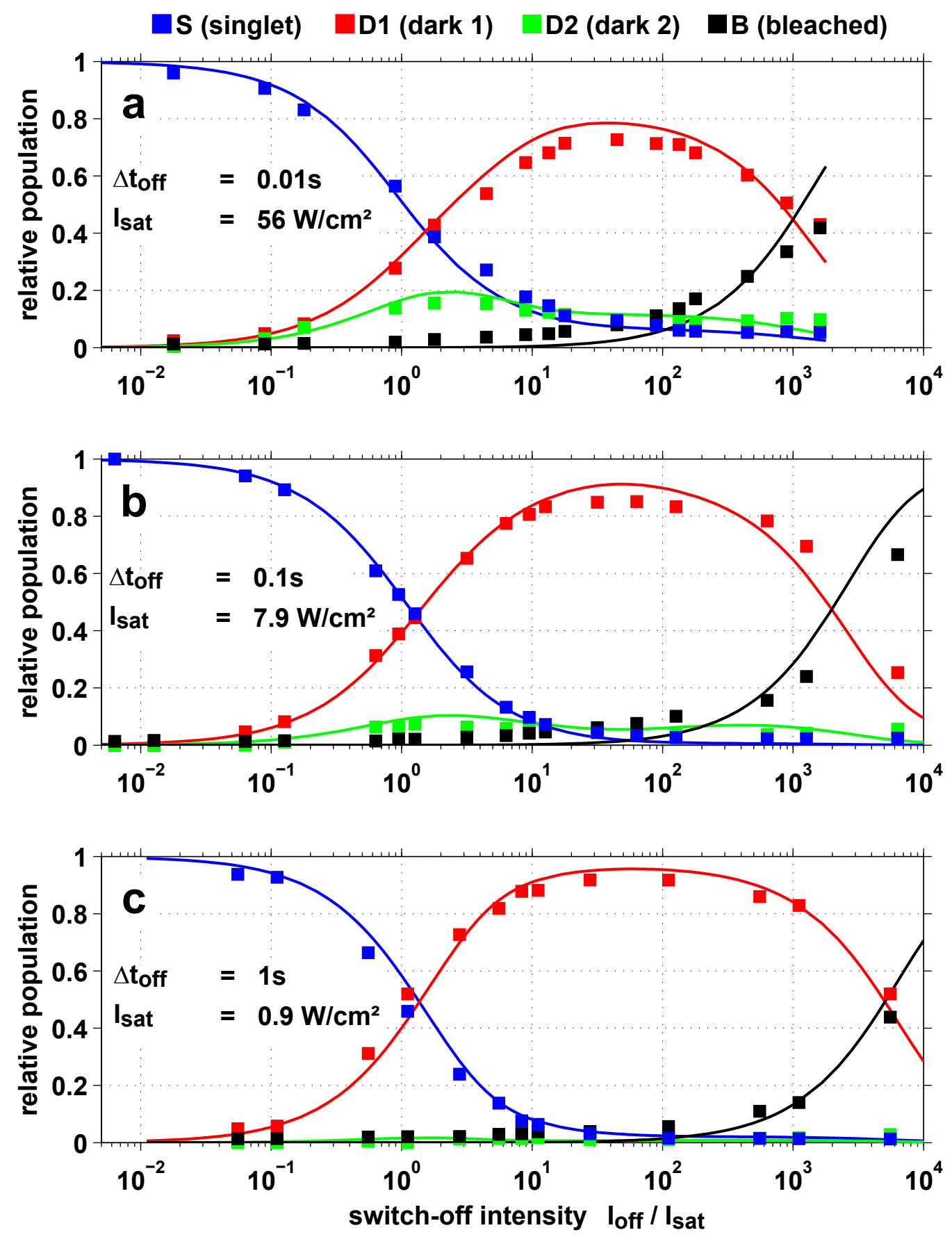

Figure 2.21: rsFastLime in PVA: intensity dependence of the fraction of molecules in state $S$ (singlet, blue), $D 1$ (stable dark state, red), $D 2$ (short lived dark state, green) and $B$ (irreversibly bleached, black) under 491nmillumination. The measurement was performed as illustrated in figure 2.20 a-c correspond to different values of the switch-off time: $\mathbf{a} \Delta t_{\text {off }}=0.01 \mathrm{~s}, \mathbf{b} \Delta t_{\text {off }}=0.1 \mathrm{~s}, \mathbf{c} \Delta t_{\text {off }}=1 \mathrm{~s}$. Intensities are given in units of the saturation intensity $I_{\text {sat }}$, the relevant resolution measure in RESOLFT microscopy. The data indicate that photoswitching of rsFastLime from the fluorescent state $S$ (blue) to the stable dark state $D 1$ (red) is most efficient when employing long switch-off times and low absolute intensities as photobleaching and the population of the short-lived dark state $D 2$ (green) are minimal in this case. Photobleaching occurs predominantly via the stable dark state $D 1$ (red) which restricts the suitability of rsFastLime as a RESOLFT marker. The photophysical model used to fit the data is outlined below. 

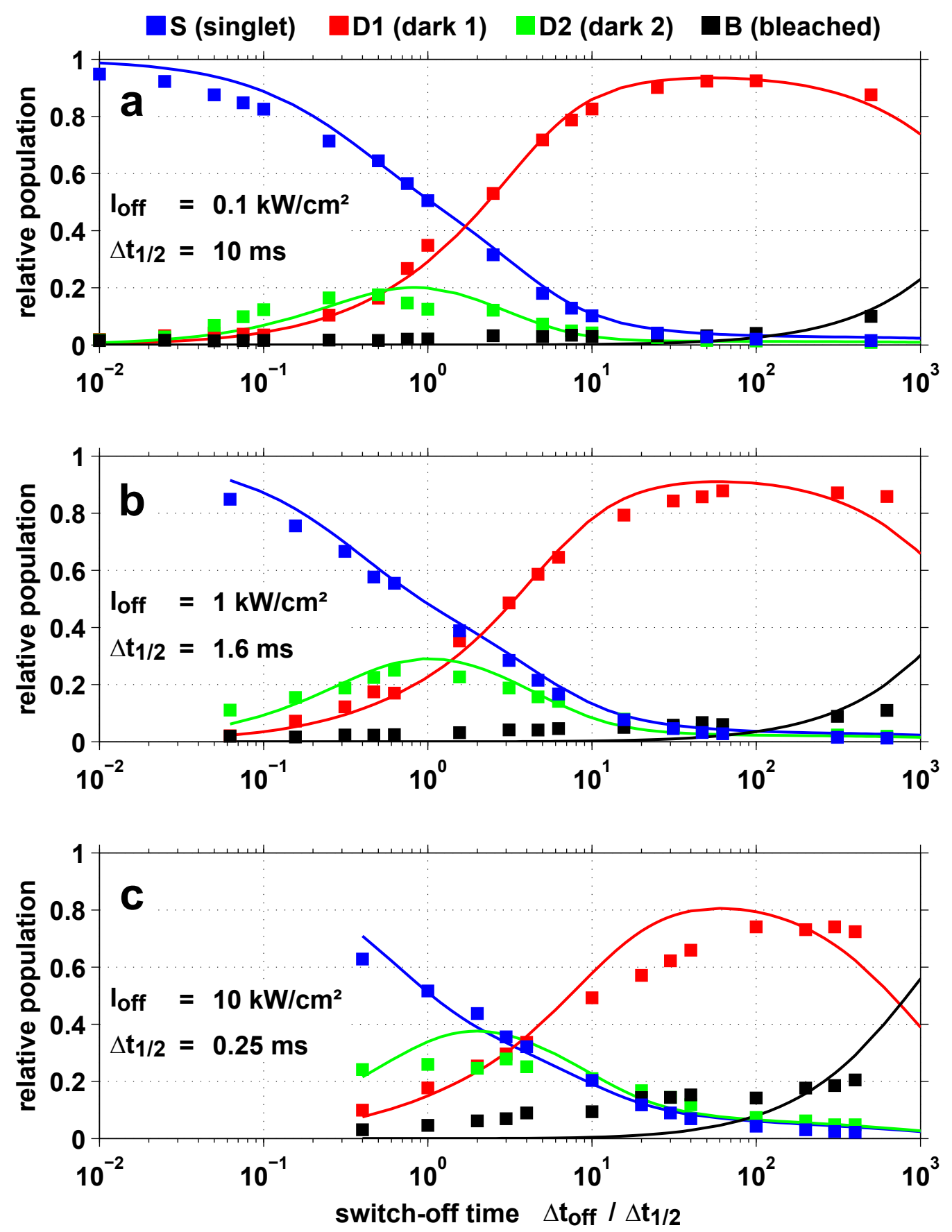

Figure 2.22: rsFastLime in PVA: time evolution of the fraction of molecules in state $S$ (singlet, blue), $D 1$ (stable dark state, red), $D 2$ (short lived dark state, green) and $B$ (irreversibly bleached, black) under $491 \mathrm{~nm}$ illumination. The measurement was performed as illustrated in figure 2.20 a-c correspond to different values of the $491 \mathrm{~nm}$ switch-off intensity: a $I_{491}=0.1 \mathrm{~kW} / \mathrm{cm}^{2}, \mathbf{b} I_{491}=1 \mathrm{~kW} / \mathrm{cm}^{2}$, c $I_{491}=10 \mathrm{~kW} / \mathrm{cm}^{2}$. The switch-off time is given in units of $\Delta t_{1 / 2}$, which denotes the time after which the fluorescent state $S$ (blue) is depleted by $50 \%$. Photoswitching from $S$ (blue) to $D 1$ (red) is most efficient at low switch-off intensities. The photophysical model used to fit the data is outlined below. 


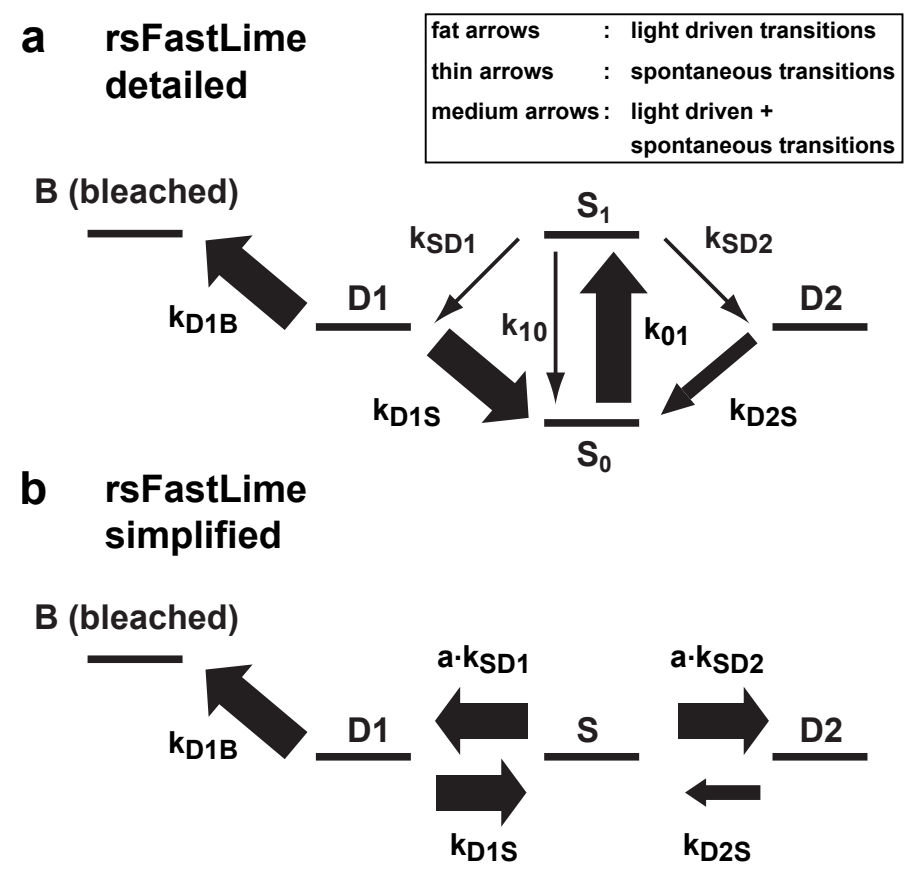

Figure 2.23: Photophysical model of the RSFP rsFastLime under 491nm irradiation. a Schematic energy level diagram. b Simplified diagram: since $k_{10}$ is larger than all other rates by several orders of magnitude the singlet system can be assumed to be in a steady state. The parameter $a=S_{1} /\left(S_{0}+S_{1}\right)=k_{01} /\left(k_{10}+k_{01}+k_{S D 1}+k_{S D 2}\right)$ denotes the (intensity dependent) fraction of singlet state molecules in $S_{1}$. Both the stable dark state $D 1$ and the short-lived dark state $D 2$ are populated via the first excited singlet state $S_{1}$. Clearly, rsFastLime is not a perfect photoswitch: due to the non-zero rates $k_{D 1 S}$ and $k_{D 2 S}$ the fluorescent singlet state cannot be fully depopulated. In addition, a fraction of molecules is irreversibly bleached (state $B$ ). Not shown: molecules in $D 1$ can effectively be recovered to the fluorescent singlet system through UV irradiation.

\section{Photophysical Model of rsFastLime}

Based upon the measurements displayed in figures 2.21 and 2.22 a model describing the photophysics of the RSFP rsFastLime under 491nm illumination has been established which can be viewed as an extension of the simple two state model introduced earlier (see figure 2.7). The extended version of this scheme incorporates the additional short lived dark state $D 2$ and the bleached state $B$ and is reminiscent of a model proposed for the RSFP Dronpa [70, 85]. It must be mentioned however that this more elaborate scheme still represents a relatively crude approximation to reality. For it can be expected that the intricate structure made up of the protein chromophore, neighboring amino acids, the hydrogen bonding network within the protein beta barrel and the various interactions between these constituents gives rise to a more complex system of molecular states [86, 87].

Figure 2.23 features the energy level diagram along with the transition rates between the different states. Rate constants bear as a subscript the identifiers of the molecular states they connect, i.e. the rate associated with the transition $A \rightarrow B$ is denoted $k_{A B}$. A light induced transition can be written in the form $k_{A B}=\sigma_{A B} \gamma I$, with $\sigma_{A B}, \gamma$ and $I$ denoting, respectively, the absorption cross section measured in $\mathrm{cm}^{2}$, the inverse photon energy measured in $\mathrm{J}$ and 
the excitation intensity measured in $\mathrm{W} / \mathrm{cm}^{2}$. For clarity, light driven and spontaneous transitions are highlighted by fat and by thin arrows, respectively. The rate $k_{D 2 S}$ must have both a spontaneous as well as a light driven component as the existence of a purely spontaneous component would entail an increase in the $D 2$ population with rising intensity, contrary to what is observed in figure 2.21. The corresponding rate $k_{D 2 S}$ is indicated by a medium fat arrow.

Figure 2.23 a features a detailed model in which the singlet states $S_{0}$ and $S_{1}$ are depicted separately. The system can be somewhat simplified when taking into account that for moderate excitation intensities of $\approx \mathrm{kW} / \mathrm{cm}^{2}$ the rate $k_{10}$ of spontaneous fluorescence emission exceeds all other rates by several orders of magnitude $\left(k_{10} \approx 10^{9} 1 / \mathrm{s}\right)$. As a consequence $S_{0}$ and $S_{1}$ can be assumed to be in equilibrium at every instant. This is tantamount to saying that $S_{1}=a S$ and $S_{0}=(1-a) S$, where $S:=S_{0}+S_{1}$ is the total singlet population and $a=S_{1} /\left(S_{0}+S_{1}\right)=k_{01} /\left(k_{10}+k_{01}+k_{S D 1}+k_{S D 2}\right)$ is the fraction of singlet state molecules in $S_{1}$. With this approximation the number of independent states is reduced by one. Figure $2.23 \mathrm{~b}$ shows the resulting simplified scheme. Its dynamics is described by the following set of independent first order differential equations

$$
\begin{aligned}
& \dot{S}=-a\left(k_{S D 1}+k_{S D 2}\right) \quad S+k_{D 1 S} \quad D 1+k_{D 2 S} \quad D 2 \\
& \dot{D} 1=a k_{S D 1} S-\left(k_{D 1 S}+k_{D 1 B}\right) \quad D 1 \\
& \dot{D} 2=\quad \begin{array}{lll}
k_{S D 2} & S & -
\end{array}
\end{aligned}
$$

along with the additional condition

$$
S+D 1+D 2+B=1
$$

embodying particle conservation. Employing matrix notation the system (2.14) can be more compactly written as

$$
\dot{\mathbf{x}}=\mathbf{A x},
$$

where $\mathbf{x}$ designates the state vector $(S, D 1, D 2)^{T}$ and $\mathbf{A}$ the coefficient matrix on the right hand side of (2.14). To fit the data shown in figures 2.21 and 2.22 the solution of 2.14) was calculated by numerically computing the expression

$$
\mathbf{x}(t)=\exp (\mathbf{A} t) \cdot \mathbf{x}_{\mathbf{0}}
$$

Here, $\exp (\mathbf{A} t)$ is definded as the exponential series $\sum_{j=0}^{\infty}(\mathbf{A} t)^{j} / j$ ! and $\mathbf{x}_{\mathbf{0}}$ is the state vector $(S, D 1, D 2)^{T}$ at time $t=0$. Since in the experiment all molecules were initially in the fluorescent state $S$ (see figure 2.20, $\mathbf{x}_{\mathbf{0}}=(1,0,0)^{T}$ was used. For the obtained fitting parameters please see appendix A.2.

\subsection{Single Molecule Switching (SMS) Microscopy with rsFastLime}

In the previous section it was demonstrated that the reversible photoswitching mechanism of rsFastLime allows for its use as a marker in super-resolution RESOLFT microscopy. To 
prove the feasibility of this concept the endoplasmatic reticulum of fixated mammalian cells expressing rsFastLime was imaged with subdiffraction resolution.

Whereas RESOLFT microscopy rests on the photoswitching of molecular ensembles, rsFastLime can be employed also for subdiffraction imaging techniques which are based on the switching and recording of individual markers. This can be achieved by transferring only a small number of isolated switchable fluorescent labels to the bright state at a time. Upon excitation these activated markers may then emit a large number of photons while neighboring emitters remain dark. Imaging the bursts of emitted photons on a pixelated detector allows to determine the positions of individual molecules with subdiffraction precision. Provided the images of different emitters on the detector do not overlap the achieved localization uncertainty $\Delta r^{\prime}$ is given by

$$
\Delta r^{\prime}=\frac{\Delta r}{\sqrt{N}}
$$

where $\Delta r$ denotes the FWHM of the point spread function of the detection optics and $N$ the number of detected photons [29, 88, 89, 90]. Following fluorescence readout markers are bleached or switched back to the dark state to allow the activation and localization of another sparse subset of fluorescent labels. Finally, the single molecule positions determined over a large number of imaging cycles can be assembled to form a super-resolution image. Since a single molecule in the on-state already constitutes the smallest conceivable focal spot no additional quenching is necessary to further reduce the size of the focal volume. In other words, in order to be imaged with subdiffraction precision an individual emitter needs to undergo no more than one switching cycle (off-on-off). This is in contrast to RESOLFT microscopy where an individual emitter has to undergo a number of cycles until its fluorescence contributes to the final image.

The first implementations of Single Molecule Switching (SMS) microscopy named PALM[35], STORM[43] and FPALM[42] used photoactivatable fluorescent proteins (PAFPs) such as PA-GFP[36], EosFP[39] and Kaede[40] or donor-acceptor pairs of cyanine dyes in conjunction with an oxygen scavenging system [45, 46] as photoswitchable markers. In all of these initial works two different wavelengths were employed for fluorescence activation and -excitation; the excitation laser also served to either switch molecules back to the dark state or to bleach them irreversibly. Activation, fluorescence excitation and image acquisition were carried out in a synchronized fashion with a single imaging cycle typically extending over a period of $\approx 1 s$. Since the formation of meaningful pictures typically required the acquisition of 10000-50000 individual images, total recording times of up to several hours were reported, putting particular strain on setup stability. Also, total internal reflection fluorescence (TIRF) microscopy was used to reduce background noise thus limiting the technique to the imaging of thin sections and surfaces.

\section{The PALMIRA Principle}

In the following it shall be demonstrated that using the RSFP rsFastLime as a switchable marker the above mentioned restrictions were overcome. Specifically, acquisition times 

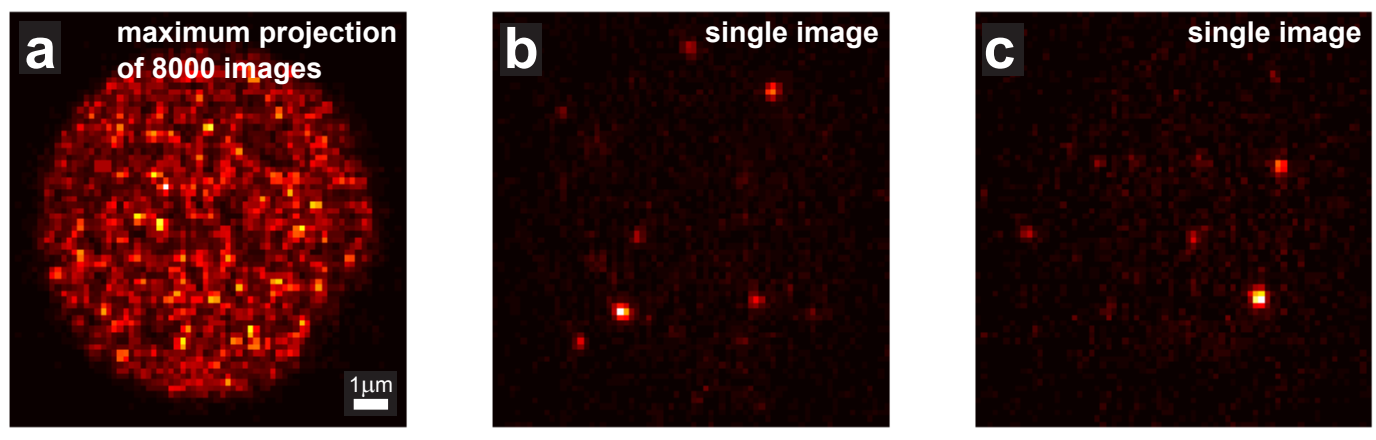

Figure 2.24: Only a fraction of $\approx 1 \%$ of rsFastLime molecules are in the fluorescent state at a time when illuminated with $491 \mathrm{~nm}$ light. a The maximum projection of 8000 camera frames reveals the density of the rsFastLime sample; the round shape represents the homogeneous illumination spot. b+c In a single camera frame only a small number of isolated emitters is visible at a time as the majority of markers reside in the dark state. Images taken at different times show different subsets of molecules in the on-state indicating that emitters are activated randomly. Single molecule images were recorded at an intensity of $I_{491}=5 \mathrm{~kW} / \mathrm{cm}^{2}$ and with a camera frame time of $\Delta t_{C C D}=2 \mathrm{~ms}$.

could be reduced $\approx 100$-fold by employing an asynchronous recording scheme utilizing a single laser and a freely running CCD camera. In addition, background noise was successfully suppressed rendering the use of TIRF microscopy unnecessary and enabling imaging from within the interior of nonsliced cells.

The basic principle of how the RSFP rsFastLime can be used as a marker in SMS microscopy is illustrated in figure 2.24. The purified protein embedded in a film of PVA was continuously illuminated with a homogeneous spot of $491 \mathrm{~nm}$ light at an intensity of $I_{491}=5 \mathrm{~kW} / \mathrm{cm}^{2}$. Simultaneously, the fluorescence signal emitted from the sample was imaged on a freely running CCD camera acquiring data with an exposure time of $2 \mathrm{~ms}$ and operating at $97 \%$ duty cycle. With these settings a total of 8000 images was taken within $16 \mathrm{~s}$.

The maximum projection of this series is shown in figure $2.24 \mathrm{a}$. It illustrates the distribution of all rsFastLime markers imaged during the 16s recording interval. By contrast, figures $2.24 \mathrm{~b}+\mathbf{c}$ feature single camera frames representing $2 \mathrm{~ms}$ snapshots of the sample taken at different times. They lucidly reveal that only a small fraction of the complete ensemble of rsFastLime molecules reside in the fluorescent state at a time. Indeed, the density of concurrently activated fluorophores is sparse enough to yield distinctly separated single molecule images on the CCD detector. Moreover, the subset of markers in the bright state is subject to continuous change as emitters are perpetually switched on and off at random. As a result images of the sample taken at different times such as in $\mathbf{b}$ and $\mathbf{c}$ show different sets of bright emitters. It may be pointed out that this observation is in perfect agreement with the photophysical model of rsFastLime which was formulated in the previous section: since the switch-on cross sections are by at least two orders of magnitude smaller than those driving the reverse on-off-transition, only a fraction of $\approx 1 \%$ of all markers reside in the on-state at a time (see e.g. figure 2.4 or equation (2.2)). The resulting random blinking of single 


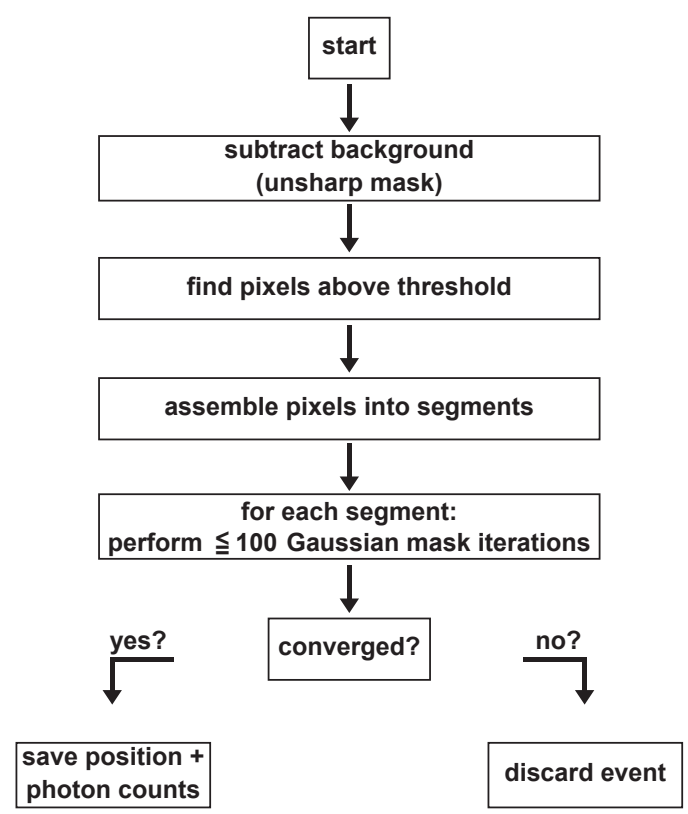

Figure 2.25: Flow diagram describing the procedure used for the determination of single molecule positions in a given camera frame. After background correction pixels with a count rate above a user-defined threshold are assembled into connected segments. These segments are then subjected to a Gaussian mask fitting routine (see text). If the algorithm does not converge within 100 iterations the corresponding burst is discarded.

molecules under constant $491 \mathrm{~nm}$ illumination with short on- and long off-times was already illustrated in figure 2.6. Thus it can assumed that every marker molecule is registered at least once if a sufficiently large number of camera frames is taken.

From this simple example it should be obvious that the recording of non-triggered, spontaneous off-on-off cycles of the switchable marker using a single laser and freely running and asynchronously operating CCD detector provides the prerequisites needed for a successful implementation of super-resolution SMS microscopy. To distinguish this imaging scheme from those SMSM techniques which are based on the synchronized activation of fluorophores it was named photoactivated localization microscopy with independently running acquisition (PALMIRA) [47, 48].

\section{Position Determination}

To determine the positions of single activated fluorophores a combined CLEAN/mask-fitting algorithm was used [91][34]. In a given camera frame a copy of the raw image was smoothed with a low-pass Gaussian filter and the result subtracted from the original data (unsharp masking or USM). This way inhomogeneities in the diffuse background could be adequately accounted for. Typically, the FWHM of the Gaussian used for smoothing the raw image was chosen to be three times that of the detection point spread function. Subsequently, image pixels with a count rate above a user defined threshold were assembled into segments of connected pixels. The exact threshold value was adjusted for each sample and set of imaging 
parameters (e.g. excitation intensity and frame time) individually. Segments consisting of at least three connected pixels above threshold were cut out together with a neighborhood of user defined size and subjected to a mask-fitting localization routine [34]. The localization algorithm operated in the form of a fixed point iteration and was equivalent to a Gaussian least-squares fit. (Although theory predicts an Airy disk for the point-spread function, a Gaussian is more tractable mathematically; for practical purposes the differences between the two are minor.) The starting point $\mathbf{r}_{0}$ of the iteration was set to the center of mass of the data passed to the routine. In each following step the data was multiplied by the PSF centered at $\mathbf{r}_{n-1}$ (the 'mask'); the new estimate $\mathbf{r}_{n}$ was given by the center of mass of the 'masked' data. Usually, the routine converged after a few iterations. In this case the retrieved position was tabulated together with the number $N$ of photons associated with the event, given by the pixel sum over the respective Airy disc. If the algorithm did not converge after a predefined maximum number of iterations the event was discarded. The final superresolution image was formed by deviding the field of view into pixels whose size was set to the mean localization accuracy (for rsFastLime typically $\approx 15 \mathrm{~nm}$ ). Every time a molecule was located within a given image region the corresponding pixel value was incremented by one. Thus, the reconstructed image was given by a two-dimensional histogram of single molecule positions. A flow diagram describing the operation of the position determination routine is depicted in figure 2.25 .

\section{PALMIRA Imaging}

The experimental setup used for PALMIRA imaging was identical to the one employed for spectroscopic measurements (see figure 2.3). Its principle components were a blue $491 \mathrm{~nm}$ laser creating in the sample an excitation spot of typically $\approx 10-20 \mu \mathrm{m}$ in diameter and an electron multiplying CCD camera used for fluorescence detection. If required the blue excitation laser could be complemented by a second ultra violet laser for additional fluorescence activation. The magnification $V$ of the detection optics was chosen in such a way as to yield an effective pixel size $a$ that was slightly smaller than the width $\Delta r$ of the PSF [34]. The parameters used were $V=150, a=160 \mathrm{~nm}$ and $a / \Delta r \approx 0.7$. Thus, the tunable experimental parameters were 1) the excitation intensity $I$ and 2) the frame rate $f$ (measured in frames per second) of the freely running CCD detector.

Generally, the goal in PALMIRA imaging is the acquisition of an image with the highest possible resolution at maximum speed and with the highest possible signal-to-noise ratio. Whereas the resolution of the reconstructed image is determined by the unambiguous assignment of detected photons to single molecules, the imaging speed is dictated by the number of molecular positions retrieved in a given time interval, i.e. by the switch-on rate, which is proportional to the applied intensity. Importantly, the density $\rho_{o n}$ of activated emitters in a given camera frame must be low enough to ensure that photon bursts from different molecules do not overlap on the detector, as otherwise precise localization of the respective markers would be compromised.

The mean number of photons emitted by an individual molecule before it is switched off to the dark state or irreversibly bleached, i.e. the achievable resolution, is a constant for moderate excitation intensities $I$. More precisely, the number $N$ of photons radiated by a single 

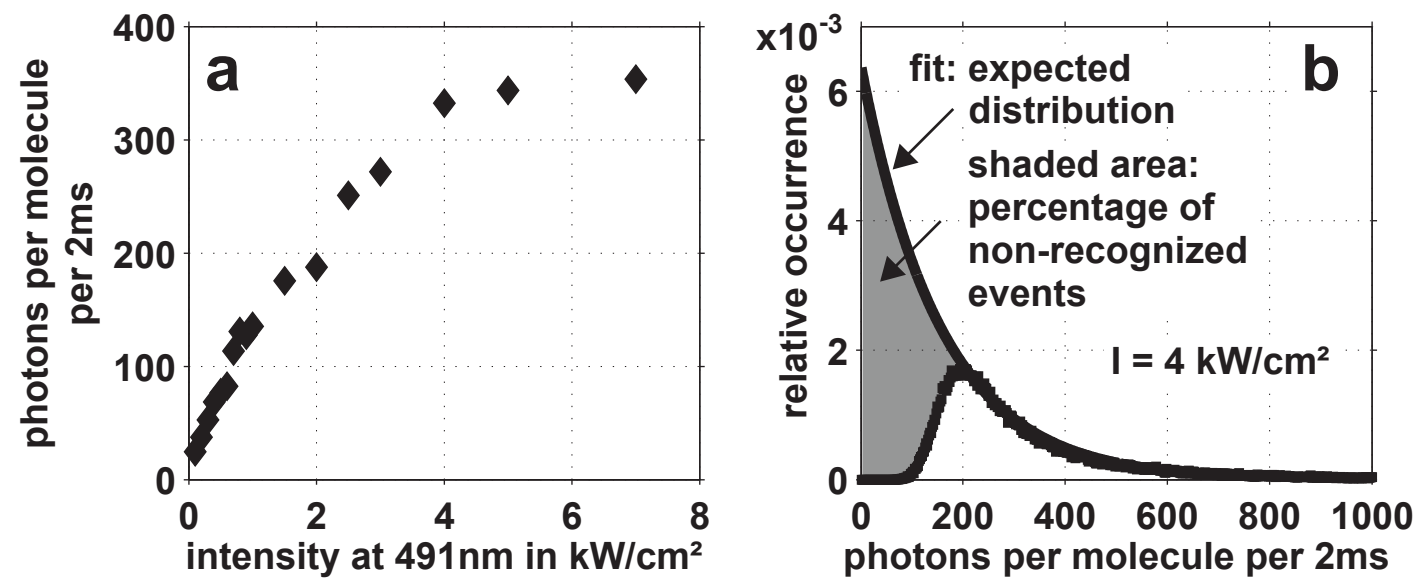

Figure 2.26: Photon statistics of single rsFastLime molecules in a film of PVA. a Mean number of detected photons per $2 \mathrm{~ms}$ camera frame plotted versus the excitation intensity $I$. The mean number of photons emitted per molecule is approximately constant. However, enhancing $I$ leads to an increase in the number of photons detected per $2 \mathrm{~ms}$ time window until at a value of $I \approx 4 \mathrm{~kW} / \mathrm{cm}^{2}$ the burst duration $T_{\text {on }} \propto 1 / I$ gets shorter than the $2 \mathrm{~ms}$ camera frame time. b Relative occurrence of the number of detected photons for an excitation intensity of $I=4 \mathrm{~kW} / \mathrm{cm}^{2}$. The expected photon histogram follows a geometric distribution (fit). However, the localization routine does not recognize events with a photon number below a certain threshold (shaded area).

emitter follows a geometric distribution $p(N)=\phi(1-\phi)^{N}$, where $\phi$ denotes the probability for switching-off or photobleaching (see (2.1)). By contrast, the average time a molecule spends in the on-state, $T_{\text {on }}$, can be tuned via the excitation intensity: $T_{\text {on }} \propto 1 / I$. Hence, the imaging speed can be substantially increased by concurrently enhancing the camera frame rate $f$ and the excitation intensity $I$ such that $T_{o n} \approx 1 / f$. Longer on-times spread bursts over several camera frames, thus increasing readout noise. On the other hand, higher intensities, which result in shorter on-times, lead to an effective increase in the number of visible molecules per camera frame because the switch-on rate is proportional to the applied intensity; for densely labeled samples this may entail the overlapping of the images of nearby molecules on the detector. In practice, the highest possible frame rate $f$ was chosen $\left(f_{\max }=500 \mathrm{~Hz}\right)$ and the intensity adjusted in such a way as to yield the maximum number of photons per molecule and camera frame.

The result of an experiment carried out to determine the optimal value of the excitation intensity $I$ for PALMIRA imaging with rsFastLime is depicted in figure 2.26a. A film of PVA containing purified rsFastLime protein was illuminated with $491 \mathrm{~nm}$ light of different intensities. For every intensity value several series of typically 10000 individual camera images were taken at different sites in the sample. A camera exposure time of $2 \mathrm{~ms}$ was chosen, corresponding to the highest possible frame rate of $f=500 \mathrm{~Hz}$. At this value of $f$ the detector was still operating at $97 \%$ duty cycle. Analysis of the data was carried out as outlined above (see 2.25. The total number of photons emitted per molecule was approximately constant, irrespective of the excitation intensity. However, at lower intensity values a single burst was 
spread over several camera frames, resulting in a smaller number of detected photons per $2 \mathrm{~ms}$ time window. As the intensity was increased fluorescence excitation was accelerated. As a result, the number of photons registered per molecule and per camera image went up until the point where the typical burst duration $T_{o n} \propto 1 / I$ was reduced to the $2 \mathrm{~ms}$ camera frame time. For rsFastLime this point was reached at $I \approx 4 \mathrm{~kW} / \mathrm{cm}^{2}$. Enhancing the applied intensity above this point did not yield more detected photons per molecule.

\section{PALMIRA Microscopy of Biological Samples}

To demonstrate that PALMIRA microscopy can be successfully applied to the super-resolution imaging of biological samples $\alpha$-tubulin filaments of intact PtK2-cells were immunostained with rsFastLime, embedded in PVA and mounted on a microscope cover slip. For data recording a sample area of $\approx 10 \mu \mathrm{m}$ in diameter was homogeneously illuminated with $491 \mathrm{~nm}$ light at an excitation intensity of $I=4 \mathrm{~kW} / \mathrm{cm}^{2}$. The frame rate of the freely running CCD detector was $f=500 \mathrm{~Hz}$ (corresponding to an exposure time of $1 / f=2 \mathrm{~ms}$ ).

Figure 2.27 features camera images number 100 (top) and 5000 (bottom) out of a total of 30000 recorded frames. In addition to the raw data $(\mathbf{1 a + 2 a})$ the figure displays the background-corrected images $(\mathbf{1 b}+\mathbf{2 b})$ and the positions of individual emitters $(\mathbf{1 c}+\mathbf{2 c})$ as identified by the above described CLEAN/mask-fitting position determination algorithm. The presented data clearly reveal that using rsFastLime as a marker in PALMIRA microscopy limits the maximum admissible labeling density: on the one hand the fact that the $491 \mathrm{~nm}$ excitation light also serves fluorescence activation enables PALMIRA imaging with only a single laser source; on the other, it implies that for a given finite camera exposure time $1 / f$ the number of on-state markers visible in a single camera frame is proportional to the applied excitation/activation intensity. For densely labeled samples this means that the intensity which optimizes the signal to noise ratio for a given photon burst, i.e. for which $T_{\text {on }} \approx 1 / f$, may cause the images of nearby molecules to overlap on the CCD detector, thus compromising resolution. This situation can be observed in figure 2.271a-c. Here, the density of on-state emitters was too high to allow the unambiguous attribution of detected photons to single emitters (see in particular the highlighted areas in 1b). Although the localization routine identified the positions of single fluorescent labels, the positioning uncertainty in this case was larger than $\Delta r / \sqrt{N}$ (see (2.18)), the expression that applies to the localization of a single isolated emitter. Single fluorescent labels could be identified unequivocally only after some of them had been irreversibly bleached (see 2a-c).

In the opposite case of a low density of bright state molecules PALMIRA imaging can be accelerated by applying additional UV activation light. Alternatively, the $491 \mathrm{~nm}$ excitation intensity can be increased.

Two wide-field images of $\alpha$-tubulin in PtK2-cells along with the corresponding PALMIRA 

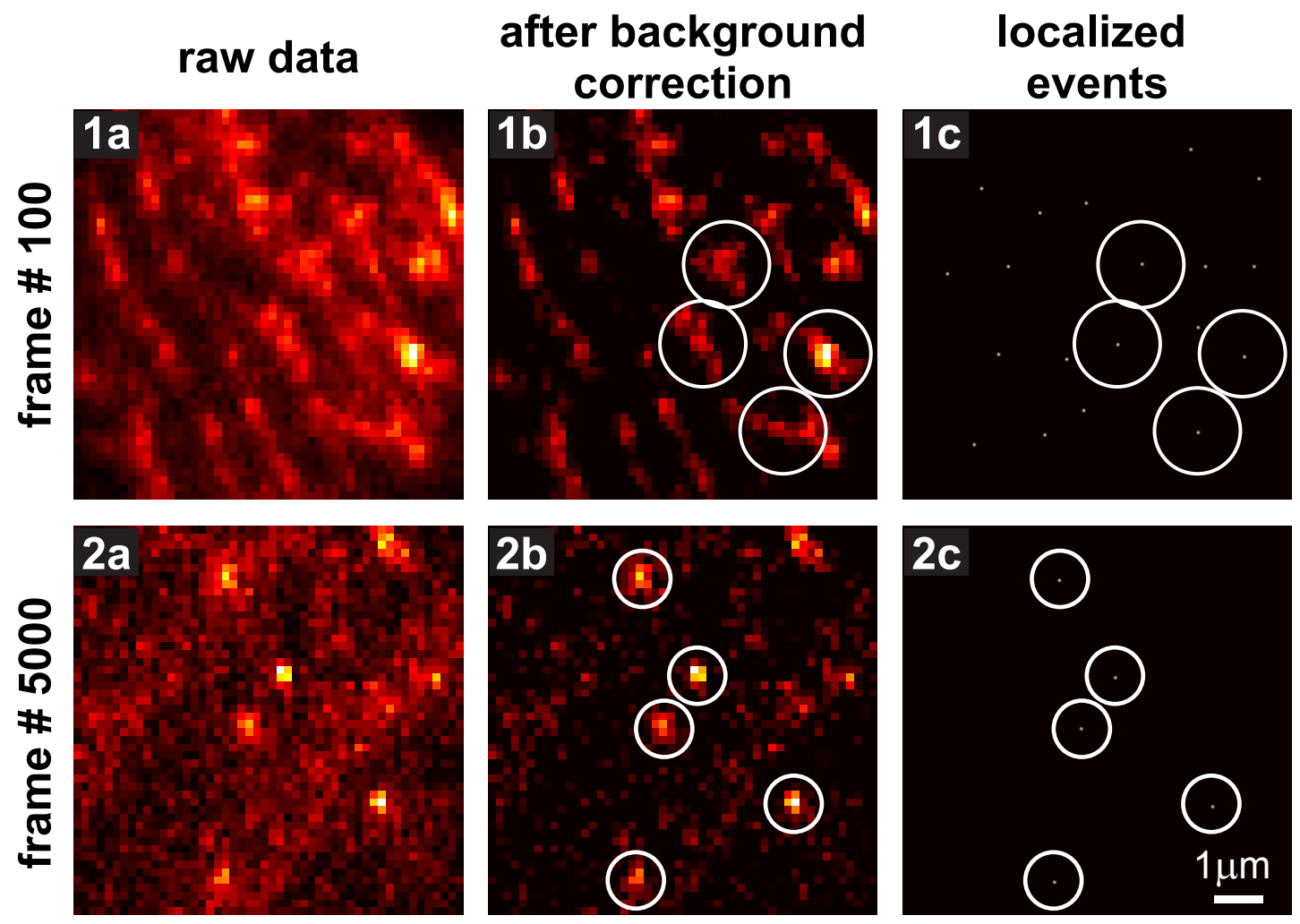

Figure 2.27: Single camera images of a PALMIRA recording of rsFastLime-stained $\alpha$-tubulin in PtK2-cells. 1a-c The high labeling density in camera image number 100 compromises resolution, as the images of nearby molecules overlap (see highlighted areas in 1b). In this case, the single molecule positions identified by the localization routine (see 1c) are likly to be erroneous. 2a-c After bleaching some of the markers, the reduced number of on-state molecules per camera frame allows the localization of single emitters with a precision that is determined by the number of detected photons.

recordings are depicted in figure 2.28 (the images shown in the bottom row correspond to the data exhibited in figure 2.27). For both images 30000 individual camera frames were recorded with a CCD exposure time of $2 \mathrm{~ms}$ and an excitation intensity of $I=4 \mathrm{~kW} / \mathrm{cm}^{2}$. The resulting total acquisition time was 60 s for both images. In the case of the image shown in the bottom row $(\mathbf{2} \mathbf{a}+\mathbf{b})$ the first 500 camera frames were discarded because the initial labeling density was too high to allow the assignment of detected photons to single emitters (see figure 2.27). Wide-field images were formed by adding up all background-corrected camera frames. The rather large effective pixel size of $160 \mathrm{~nm}$ used for data recording adds to the coarse appearance of the wide-field images, which should be taken into account when comparing them to their PALMIRA counterparts. The latter were constructed by dividing the field of view into $20 \mathrm{~nm} \times 20 \mathrm{~nm}$ pixels and incrementing the count rate of each pixel by one every time an emitter was localized within the respective sample area. The $20 \mathrm{~nm}$ pixel size chosen for the representation of PALMIRA images was approximately equal to the mean localization accuracy given by the average number of detected photons per burst 

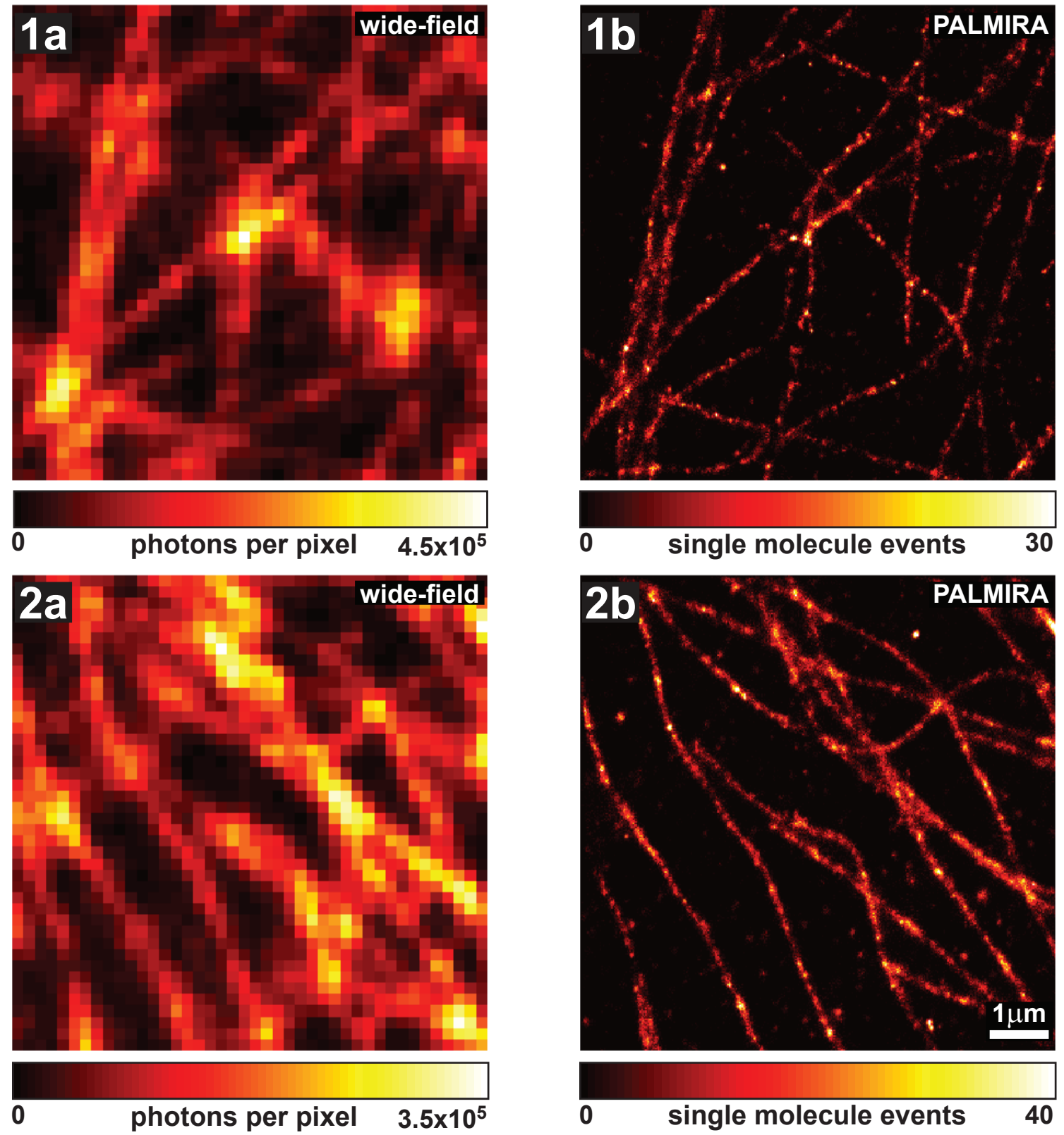

Figure 2.28: Wide-field images of $\alpha$-tubulin in PtK2-cells immunostained with rsFastLime (1a+2a) and the corresponding PALMIRA recordings $(\mathbf{1 b}+\mathbf{2 b})$. A total of 30000 camera frames was acquired for each of the two images. The intensity of the $491 \mathrm{~nm}$ excitation light was $I=4 \mathrm{~kW} / \mathrm{cm}^{2}$ and a CCD exposure time of $2 \mathrm{~ms}$ per frame was used, resulting in a recording time of 60s per image. Wide-field images were constructed by adding up all background-corrected frames. PALMIRA images were formed by dividing the field of view into $20 \mathrm{~nm} \times 20 \mathrm{~nm}$ pixels and incrementing the count rate by one each time a single molecule event was localized in the respective area. 


\section{$(<\Delta r / \sqrt{N}>\approx 15 \mathrm{~nm})$}

The excellent spatial resolution achieved in the PALMIRA images is immediately obvious; they clearly reveal details which remain hidden in the corresponding wide-field recordings. The observed thickness of $\approx 70 \mathrm{~nm}$ of the combined construct made up of tubulin strand, antibody and protein label agrees well with results obtained by electron microscopy [92]. A noteworthy feature exhibited particularly in the PALMIRA image shown in figure $2.281 \mathbf{b}$ is the relatively inhomogeneous density of detected single molecule events along several of the illustrated tubulin strands. A possible explanation for this finding is a non-uniform distribution of fluorescence labels on the respective microtubules. Alternatively, it could have been caused by the suppression of dim single molecule events through the localization routine.

\subsection{Summary of RESOLFT- and SMS Microscopy with rsFastLime}

The use of fluorescent proteins as fluorescence labels has grown into an invaluable tool in cell biology as it allows the endogenous expression of markers in living cells [62]. Since conventional fluorescence microscopy techniques fail to resolve structural details on length scales smaller than about half the optical wavelength (typically $\approx 200 \mathrm{~nm}$ along the lateral and $\approx 500 \mathrm{~nm}$ along the axial direction), methodologies which provide resolution below this limit open up a new window into the structure and function of living organisms on the nanometer scale.

At the core of all far-field fluorescence nanoscopy techniques lies the possibility to switch the marker molecules between a fluorescent and a non-fluorescent state in order to single out bright structures of subdiffraction size and record them sequentially in time. These nanosized structures are then assembled to form the final super-resolution image [5]. Along these lines, the results presented in the preceding sections demonstrate that the existence of a bright- and a long-lived photoswitchable dark state in the RSFP rsFastLime [72] permits its use as a photoswitchable marker in both RESOLFT- and Single Molecule Switching (SMS) Microscopy.

\section{RESOLFT Microscopy}

It was demonstrated that using blue $(\lambda=491 \mathrm{~nm})$ and ultra violet light $(\lambda=405 \mathrm{~nm}) \mathrm{rs}$ FastLime can be reversibly switched between a fluorescent and a stable non-fluorescent state. Furthermore, the 491nm-induced transition from the on- to the off-state was shown to feature a strongly non-linear saturable behavior (see figures 2.10 and 2.11). Experimental evidence was provided that these characteristics of rsFastLime can be successfully combined in a RESOLFT-type microscope to enable far-field fluorescence imaging with a resolving power that is no longer limited by diffraction. Effective inhibition of fluorescence could be achieved at arbitrarily low light intensities, provided the duration of the applied switch-off pulse was sufficiently long (see figure 2.10). For switch-off times in the range of seconds saturation intensities as low as $\approx 1 \mathrm{~W} / \mathrm{cm}^{2}$ were reported. These results are of great relevance to the possible super-resolution imaging of living cells as low light intensities minimize photo stress 
of the specimen and possible radiation-induced damage; in addition, they render the use of expensive high power light sources obsolete. Since the use of low light intensities called for longer acquisition times, a parallelized wide-field setup similar to the one described in [74] was employed for RESOLFT imaging with rsFastLime. This arrangement permitted the imaging of $\approx 10 \mu \mathrm{m}$ sized areas with a resolution of $\approx 60 \mathrm{~nm}$ along one lateral direction within only a few seconds, constituting a significant reduction in recording time as compared to related RESOLFT techniques relying on point scanning data acquisition [68, 93].

In principle the resolving power of a RESOLFT microscope can be arbitrarily enhanced by increasing the intensity of the switch-off light [14]. Yet, using the RSFP rsFastLime as a fluorescence label resolution was practically limited by the insufficient reversibility of the switching transition caused by irreversible photobleaching: in RESOLFT microscopy increasing the resolving power of the microscope requires the use of higher switch-off intensities and finer scanning of the sample (Niquist criterion); consequently, an individual marker molecule is bound to undergo an increased number of switching cycles and sustain a higher photon flux until its fluorescence contributes to the final image [5]. The raw image presented in figure 2.182b shows the implications this effect may have on the recorded data. Photobleaching effectively also limited imaging speed as shorter switch-off times necessitated the application of higher intensities in order to achieve the same resolution (see figure 2.10 c). However, photodestruction generally is a non-linear effect (it occurs predominantly via excited states) [94] and hence the application of higher intensities results in enhanced photobleaching rates even if the total photon number remains constant.

In the experiments presented here, the attainable resolution was also compromised by instabilities of the Mach-Zehnder interferometer, which lead to imperfections of the intensityzeros of the modulated illumination profile. Using a more stable device for the generation of the stripe pattern such as a diffraction grating should readily yield better results.

To lessen the detrimental effects of photobleaching in RESOLFT microscopy the number of switching cycles per molecule could be reduced by utilizing an intelligent imaging scheme [95]. E.g. in a point type scanning arrangement the fluorescence signal at a particular pixel can be checked before the switch-off beam is applied. If the collected signal falls below a certain threshold the focus can be moved on to the next pixel. This way unnecessary on- and off- switching of out-of-focus markers can be kept to a minimum. This concept has already been successfully applied in STED microscopy (unpublished data) and could well be used in RESOLFT imaging with switchable fluorescent proteins. However, it is not easily compatible with wide-field illumination, thus precluding the simultaneous use of a parallelized data acquisition arrangement.

A disadvantage of using rsFastLime for RESOLFT microscopy consists in its limited brightness. The fact that the excitation light also switches molecules to the dark state ('negative switching') implies that only a limited number of fluorescence photons can be collected from an individual molecule per switching cycle (see equation (2.1)). As a consequence of this phenomenon longer fluorescence readout leads to an effective decrease in resolution, as can be seen in figure 2.11c. This effect is further enhanced if the second, short-lived dark state is significantly populated during the application of the switch-off pulse. In this case longer readout times effectively lead to a further decrease of the fluorescence inhibition efficiency (i.e. an elevated residual fluorescence level) as molecules returning from the short-lived dark 
state contribute to the collected signal. These complications do not arise with RSFPs where the excitation light also serves fluorescence activation ('positive switching') and light of a different wavelength is used to switch chromophores to the dark state. Examples for RSFPs of this type are ASFP595 [96, 68, 74] and Padron [97].

The microscope used in this work provides resolution enhancement along one lateral dimension only. Extending parallelized wide-field super-resolution imaging to two dimensions requires a switch-off illumination profile capable of confining fluorescence emission in all directions. Ideally, such an intensity distribution should feature an array of 'doughnut' modes as utilized in point scanning RESOLFT techniques. An illumination profile which approximates such an array of 'doughnuts' can be generated by coherently overlaying two perpendicularly oriented stripe patterns. The resulting grid-like profile can provide simultaneous resolution enhancement in all lateral directions, albeit not uniformly as the effective foci created by such a grid feature a square shape.

However, 2D super-resolution imaging can be implemented with a simple 1D stripe pattern. To this end, it is necessary to perform phase scans for a number of different orientations of the fringes with respect to the sample [77, 80]. The underlying mechanism can be pictured most clearly when considering the concept of resolution enhancement in the Fourier domain where a higher resolving power corresponds to an enlargement of the region of support of the optical transfer function (OTF) of the microscope. In this picture, increasing the resolution in one dimension is equivalent to extending the OTF in reciprocal space in the respective direction. By rotating the illumination pattern in real space a complete enlarged OTF of circular shape is mapped out in the $k_{x}-k_{y}$-Fourier plane. From the images recorded with different phase values and pattern orientations a single super-resolution image can be computationally reconstructed [76, 77, 80]. Importantly, the procedure requires the exact knowledge of both the intensity distribution of the switch-off pattern and the corresponding fluorescence inhibition (or saturation-) curve. With the setup shown in figure 2.12 the orientation of the structured illumination pattern could be altered by inserting a rotatable dove prism in the intermediate image plane. Alternatively, a rotatable diffraction grating could be employed for the generation of the illumination profile [77, 80].

Although the parallelized wide-field setup used in this work enables fast image acquisition it has the disadvantage of providing no optical sectioning along the axial direction, thus effectively constricting this methodology to the imaging of two dimensional objects. This restriction can be partially circumvented by cutting the sample into thin slices and reassembling the pictures taken of the individual layers to form a three dimensional image [98]. However, such a procedure is limited to the imaging of fixed samples. Recently, three dimensional resolution doubling with structured wide-field illumination was realized utilizing a setup with three [82, 83] or six [81] interfering beams produced by combining the diffraction orders $0,+1$ and -1 from a linear diffraction grating. As in the two dimensional case, reconstruction of the final super-resolution image was carried out via frequency unmixing in Fourier space. In principle, this wide-field approach could be combined with the saturation of switchable molecular transitions [14, 79, 80, 74] to yield resolution enhancement (far) beyond a factor of two in all three dimensions. 


\section{SMS Microscopy}

SMS microscopy with rsFastLime was performed employing continuous illumination from a $491 \mathrm{~nm}$ laser and a freely running CCD camera for fluorescence detection. The rsFastLimestained microtubular network of intact PtK2 cells was successfully imaged with a resolution of $\approx 15-20 \mathrm{~nm}$ within a recording time of $\approx 60 \mathrm{~s}$. The use of only a single laser source in connection with an asynchronous image acquisition protocol (PALMIRA) [47, 48] constituted a substantial simplification of the image acquisition process as compared to previous single molecule based super-resolution microscopy techniques [35, 43, 42]. Fast data acquisition with an optimal signal to noise ratio was achieved by adapting the average duration of a photon burst to the camera exposure time via suitable choice of the excitation intensity. This way, special measures for further background reduction, such as TIRF imaging, were not required.

This improved recording scheme was made possible by the particular photophysics of the switchable marker protein rsFastLime. Specifically, illumination at $491 \mathrm{~nm}$ caused a single emitter to exhibit blinking with short on- and long off-times [70] (see also figure 2.6), implying that both on- and off-switching were driven by the $491 \mathrm{~nm}$ excitation light. As a result, only a small intensity-independent fraction of $\approx 1 \%$ of all emitters were in the bright state at a time. So long as the labeling density remained below a critical value this caused the visible markers to appear as isolated spots on the CCD detector, which subsequently enabled their localization with high precision. Since molecules were perpetually switched on and off at random, the set of recorded single molecule coordinates approached the 'true' chromophore distribution as the total number of localized events was augmented.

Resolution in SMS microscopy is determined by the number of photons detected from a single emitter. With an average number of $\approx 200$ detected photons per rsFastLime molecule the mean localization uncertainty in the measurements described here was limited to $\approx 15$ $20 \mathrm{~nm}$. Higher resolution can readily be achieved by using brighter switchable markers, such as e.g. photochromic rhodamines [53]. In addition, resolution in SMS microscopy can be tuned by displaying only those single molecule coordinates which correspond to photon bursts with a given minimum brightness [48]. Although this procedure may serve to boost the resolving power, it comes at the expense of reduced sample information since events below the threshold are discarded.

However, the problem of incomplete information about the dye distribution in the sample cannot be entirely avoided in single molecule based super-resolution techniques; indeed, it is inherent to this imaging modality. In order to be able to distinguish a single molecule event from diffuse background it is indispensable to impose a lower threshold on the number of photons in a given burst. As a result, the effective detection probability varies strongly non-linearly with the brightness of single molecules, which may lead to a distortion of the dynamic range in the reconstructed image. As a side effect, diffuse background is strongly 
suppressed, adding to the sharp appearance of the image. Thus, details of the final recording depend on the choice of parameters used for data processing.

A particular complication of PALMIRA microscopy with rsFastLime is the fact that the minimum fraction of activated markers is determined by molecular parameters and cannot be actively controlled via experimental settings. Hence, the labeling density in the sample must not exceed a certain critical value; otherwise overlapping images of nearby molecules ('double events') compromise resolution. If the initial labeling density is too high to afford PALMIRA imaging, it must be downsized by prebleaching some of the labels. However, prebleaching may induce unwanted artifacts if the bleaching rate varies within the sample. E.g. such a procedure could potentially alter the relative concentration of markers in different parts of the cell. In order to overcome these difficulties the switch-on cross talk of the excitation light must be lowered. I.e. PALMIRA imaging even of densely labeled samples is possible if the switching-on of dark-state markers by the excitation light is negligible. In the case of rsFastLime targeted mutagenesis of the protein chromophore and/or its environment might provide a remedy of this problem.

Photobleaching generally poses less of a problem in SMS microscopy than it does in RESOLFT imaging because an individual emitter needs to undergo only a single switching cycle (offon-off) in order to be recorded and localized. Generally, it is not required that an emitter be bleached after it was imaged. The statistical spread in the determined position of a single marker which is registered more than once constitutes a natural measure of the achieved localization precision. Thus, a single molecule which is imaged several times may effectively serve to elevate image contrast.

Recording times in SMS microscopy are determined by the rate at which marker molecules can be switched on, read out and switched off and by the number of single molecule positions per area needed to form a meaningful image of the object. This implies that, fundamentally, imaging speed is limited only by the fluorescence lifetime of the label and by the fact that in a given camera frame only one emitter is allowed in the bright state per diffraction zone. Hence, the recording of $10^{3}-10^{4}$ photons per millisecond should allow the localization of $\approx 1000$ molecules within a diffraction-limited area with a precision of $\approx 10 \mathrm{~nm}$ in $2 \mathrm{D}$ in just $\approx 1$ s [42, 5]. Importantly, the absolute size of the object has no bearing on the recording time, in contrast to scanning methods like RESOLFT microscopy. In terms of recording speed, the latter are advantageous when it comes to the imaging of objects which are only a few microns in size [23]. Practically, in SMS microscopy the most densely labeled sections of the sample dictate the highest admissible activation- and thus acquisition rate. 


\section{Two-Color Single Molecule Switching Microscopy}

In the previous chapter it was demonstrated that the RSFP rsFastLime can be successfully employed as a switchable label in SMS microscopy. PALMIRA recordings of the microtubular network of intact PtK2 cells yielded images with an effective resolution of down to $\approx 15-20 \mathrm{~nm}$. However, since only one type of fluorescence marker was used in these experiments they merely allowed to image the distribution of a single molecular species. Resolving the spatial organization of two or more biomolecules on sub-diffraction length scales is of great importance in a vast number of biological applications, but usually requires the use of two fluorescence labels of different color and the same number of detection channels. In the past, molecular colocalization at the nanoscale was analyzed using Förster resonance energy transfer (FRET) [99] or two-color STED microscopy [100, 101]. By contrast, this chapter discusses the first implementation of two-color PALMIRA imaging with nanoscale resolution [49] using the green emitting RSFP rsFastLime [72] and the red organic dye cyanine 5 (Cy5) [102]. By the time of writing, further implementations of two- [50] or multicolor [51, 52] nanoscale fluorescence imaging based on single molecule switching have been realized.

\subsection{Dark State Shelving of Cy5}

The prerequisite for PALMIRA imaging is the use of fluorescent labels whose transition between a dark off- and a fluorescent on-state can be controlled in such a way that only a sparse subset of the entire ensemble of markers is fluorescent at a given instant. Therefore, it is crucial to find experimental conditions which allow to transfer a sufficiently large fraction of emitters to the dark off-state and at the same time ensure the stochastic switching-on of a sub-ensemble of marker molecules. Here this concept was realized by switching the organic dye Cy5 to its triplet state $T_{1}$ or another metastable dark state and recording those molecules which returned to the fluorescent ground state $S_{0}$. Only recently it was shown that this imaging mode, termed ground-state depletion and single-molecule return (GSDIM), constitutes a general nanoscopy scheme that can be extended to a great number of standard organic fluorophores [58].

For the two-color PALMIRA experiments described in this chapter the red emitting Cy5 was chosen to ensure spectral separation from the second label used for two-color imaging, namely the green emitting RSFP rsFastLime. Cy5 can be excited at $633 \mathrm{~nm}$ and emits fluorescence peaked at around $670 \mathrm{~nm}$. Apart from inter system crossing to the triplet state, cyanine dyes are known to undergo a photo-induced trans-cis isomerization to a non-fluorescent cis 

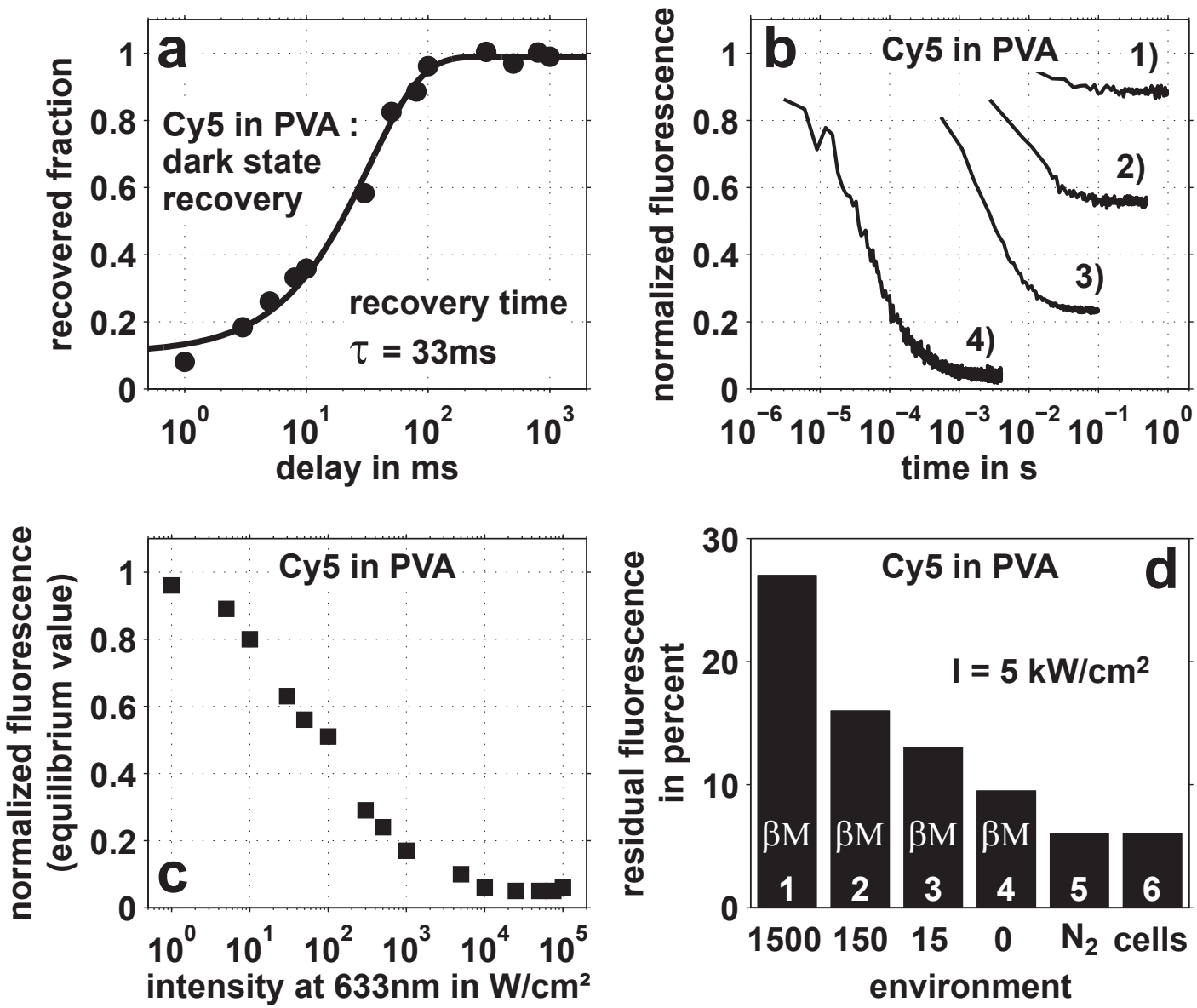

Figure 3.1: Dark state recovery and ensemble photoswitching of Cy5 molecules embedded in PVA upon illumination with $633 \mathrm{~nm}$ light. a Fluorescence recovery from the dark state after complete switch-off $\left(\Delta t_{\text {off }}=10 \mathrm{~ms}, I=10 \mathrm{~kW} / \mathrm{cm}^{2}\right)$. The experiment was performed as described in figure 2.19a. b Temporal decay of fluorescence emission due to dark state shelving, recorded at different intensities: 1) $I=5 \mathrm{~W} / \mathrm{cm}^{2}, 2$ ) $I=50 \mathrm{~W} / \mathrm{cm}^{2}$, 3) $I=500 \mathrm{~W} / \mathrm{cm}^{2}$, 4) $I=50 \mathrm{~kW} / \mathrm{cm}^{2}$. Since the switch-off light has to counteract spontaneous relaxation to the fluorescent state, the emitted fluorescence approaches an intensity-dependent equilibrium level. c Fluorescence emission at maximum dark state shelving (equilibrium level in b) vs intensity. d Dependence of Cy5 photoswitching on environmental changes. The equilibrium fluorescence level reached via illumination at an intensity of $I=5 \mathrm{~kW} / \mathrm{cm}^{2}$ is plotted for different concentrations of the triplet quencher $\beta$-mercaptoethanol ( $\beta \mathrm{M}$; bars 1-4; concentrations in milli Molar given below each bar), after nitrogen purging (bar 5) and inside PtK2 cells (bar 6). The data indicate that the triplet state is involved in Cy5 photoswitching. 
isomer [103]; Cy5 in particular is known to be photoswitchable at the single molecule level [45, 46]. As in the case of rsFastLime, the 633nm excitation light triggers the transition of $\mathrm{Cy} 5$ to the dark state, indicating that the latter is populated via the first excited singlet state $S_{1}$. Evidence for this statement is given in figure $3.1 \mathrm{~b}$. It shows the temporal decay of fluorescence emission from an ensemble of Cy5 molecules embedded in a film of PVA upon irradiation with $633 \mathrm{~nm}$ light. The four curves depicted in the figure correspond to different excitation/switch-off intensities (see figure caption). A very similar experiment was performed with the RSFP rsFastLime (see figure 2.5 a). When comparing the outcome of the measurements carried out with rsFastLime on one hand and with Cy5 on the other an important difference catches the eye:

In the case of rsFastLime, photoswitching is based on a cis-trans isomerization of the chromophore [72]. With a lifetime of several hours the dark state is stable and can be effectively reverted to the on-state by ultra violet light. The residual fluorescence level of $\approx 1 \%$ observable in figure $2.5 \mathrm{a}$ is due to a much weaker cross section for dark state activation at $488 \mathrm{~nm}$. As both fluorescence activation and de-activation occur from excited states, the residual fluorescence level is independent of the excitation intensity. (Slight deviations from this rule were shown to be related to the existence of a second, short-lived dark state.)

The situation is very different in the case of Cy5. Figure 3.1 a reveals that after switching an ensemble of Cy5 molecules embedded in a film of PVA to the dark state with $633 \mathrm{~nm}$ light, fluorescence spontaneously recovers within several tens of milliseconds. Notably, the initial fluorescence level is completely restored. This finding is in stark contrast to the behavior of rsFastLime where the bulk part of switched-off markers stay in the non-fluorescent trans state for up to several hours [72] and only a small fraction of dark state emitters show thermal relaxation on a ms-timescale (figure 2.19a). As the switch-off light has to counteract the spontaneous recovery of emitters to the fluorescent state, the percentage of $\mathrm{Cy} 5$ molecules which can be shelved in the dark state at a time depends on the intensity of the applied 633nm illumination. This phenomenon can be observed in figure 3.1 b which features the fluorescence signal emitted from an ensemble of Cy5 molecules embedded in PVA and subjected to $633 \mathrm{~nm}$ excitation light of different intensities (curves 1-4: $I=5,50,500,50000$ $\mathrm{W} / \mathrm{cm}^{2}$, respectively). All curves are normalized to the level of fluorescence emission at time $t=0$, i.e. before switch-off. Upon irradiation fluorescence decreases until it reaches a certain equilibrium value which is a function of the applied intensity. Figure 3.1c features the equilibrium fluorescence level plotted versus the intensity of the $633 \mathrm{~nm}$ excitation light over a wide range of intensity values. Finally, It could be observed that dark state shelving is much more efficient in environments deprived of molecular oxygen, e.g. by nitrogen purging, and deteriorates in the presence of the triplet quencher $\beta$-mercaptoethanol. These findings are tabulated in figure $3.1 \mathrm{~d}$. They give a strong indication that the triplet state $T_{1}$ is involved in the photoswitching of Cy5.

Presumably due to its low permeability for molecular oxygen [104] the mounting medium PVA made it possible to switch about $94 \%$ of all markers to the dark state at a given time. Likely, the remaining $6 \%$ are due to a switch-on crosstalk which can be attributed to a light driven multi-step recovery of the dark state through electronically higher excited states [105]. 


\subsection{Two-color Imaging of Biological Samples}

Owing to the switch-on crosstalk, irradiation with 488 and $633 \mathrm{~nm}$ light forms a dynamic equilibrium which transfers the majority of emitters to the dark state. On the single-molecule level this equilibrium manifests itself as a reversible blinking of individual rsFastLime and Cy5 molecules brought about by repeated switching between the fluorescent and the nonfluorescent state. Fluorescence time traces of single emitters are shown in figure 3.2. Sparse distributions of either rsFastLime or Cy5 molecules embedded in PVA were exposed to 488 (rsFastLime) or $633 \mathrm{~nm}$ light (Cy5) and the emitted fluorescence imaged on a freely running CCD camera operating at a frame rate of $f=(2 \mathrm{~ms})^{-1}$. The applied intensities were $I_{488}=4 \mathrm{~kW} / \mathrm{cm}^{2}$ and $I_{633}=10 \mathrm{~kW} / \mathrm{cm}^{2}$.

Single rsFastLime fluorophores featured a homogeneous blinking behavior with on- and off-times in the order of milliseconds and seconds, respectively (figure 3.2c-e). By contrast, individual $\mathrm{Cy} 5$ molecules exhibited noticeable differences in their blinking characteristics. While some showed uniform millisecond on- and longer off-times (f) others featured constant fast on-off-on transitions (g), although the fraction of labels with this signature was rather small. Yet another group of emitters exhibited both types of blinking properties (h). Presumably, this kind of variable blinking behavior is caused by heterogeneities in the PVA environment which translate into local differences of the triplet quenching rate [104]. In summary, $\approx 99 \%$ of all rsFastLime and $\approx 94 \%$ of all Cy 5 molecules could be switched to the dark state at a given instant enabling the recording of single isolated molecules in densely labeled samples.

Importantly, no photoswitching or fluorescence emission was observed from Cy5 when reading out rsFastLime or vice versa. In the case of Cy5 this result seems to contradict earlier reports which stated that this fluorophore could be switched from the dark cis to the fluorescent trans isomer via illumination at 532nm or at shorter wavelengths [45, 46]. However, those experiments studied Cy5-photoswitching under very different environmental conditions, namely in a medium deficient in molecular oxygen and enriched with large concentrations of a triplet quenching reagent.

Two-color PALMIRA imaging was performed on a setup identical to the one depicted in figure 2.3, now equipped with a $488 \mathrm{~nm}$ and a $633 \mathrm{~nm}$ excitation laser. Both beams were superimposed in the sample to yield homogeneous illumination spots of $\approx 10 \mu \mathrm{m}$ in diameter. As there was no crosstalk between photoswitching or fluorescence readout of Cy5 and rsFastLime, separation of their respective fluorescence signals could be achieved either by sequential excitation or by simultaneous illumination with 488 and $633 \mathrm{~nm}$ light and spectral separation of the emitted fluorescence. Here, the sample was first illuminated with $633 \mathrm{~nm}$ light to image the distribution of Cy5 labels; subsequently, the rsFastLime-stained structures were recorded by applying $488 \mathrm{~nm}$ irradiation. Since the same detection path was used in both cases, the fluorescence filter in that path was exchanged after recording of Cy5 was completed. As described in the previous chapter, a CCD detector operating at the maximum 

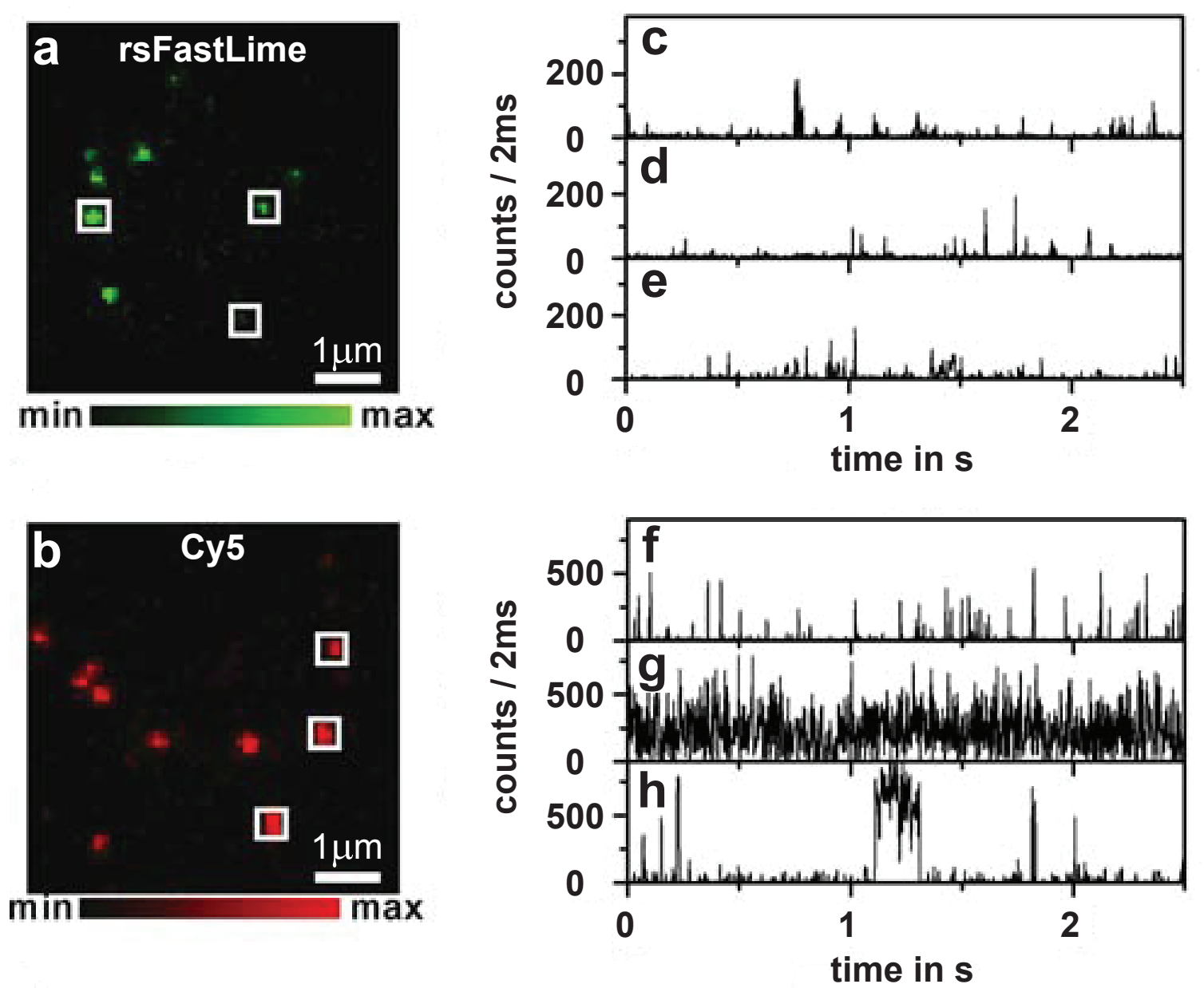

Figure 3.2: Irradiation with 488 and $633 \mathrm{~nm}$ light causes strong reversible photoblinking of single rsFastLime and Cy5 molecules in PVA, respectively. Blinking is caused by multiple transitions between bright on- and dark off-states. The CCD images are snapshots of single rsFastLime (a) and Cy5 (b) molecules scattered in a PVA film (scale bar $=1 \mu \mathrm{m}$ ). Wheareas photoblinking is homogeneous for rsFastLime (c-e), the blinking characteristics differ between single Cy5 molecules, ranging from ₹millisecond on- and longer off-times (f) to bright molecules with very short off-times (g) and emitters switching between these blinking modes (h). At intensities of $4 \mathrm{~kW} / \mathrm{cm}^{2}$ of $488 \mathrm{~nm}$ and $10 \mathrm{~kW} / \mathrm{cm}^{2}$ of $633 \mathrm{~nm}$ light up to $\approx 200$ and $\approx 700$ photons are detected from single rsFastLime and Cy5 molecules within a $2 \mathrm{~ms}$ camera frame, respectively. 


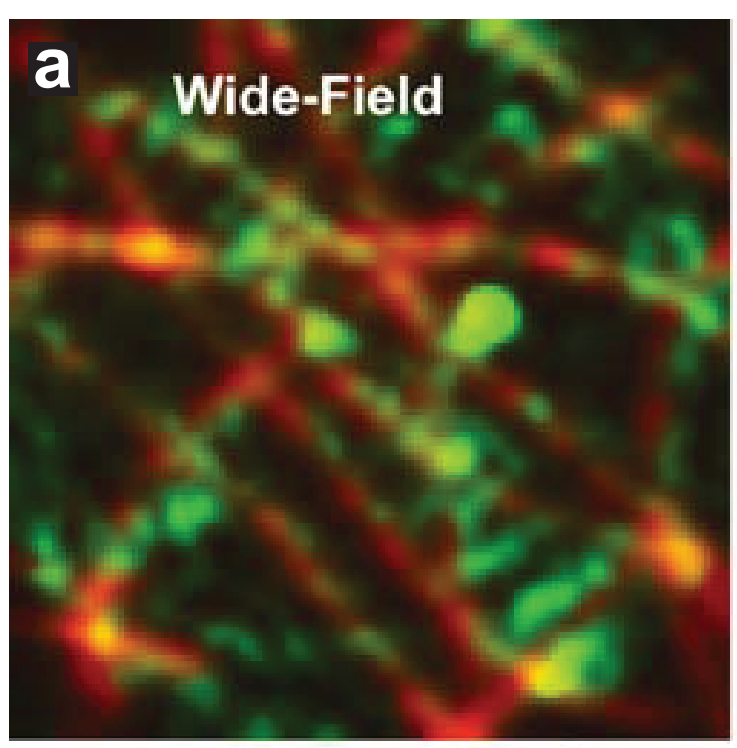

$\min$

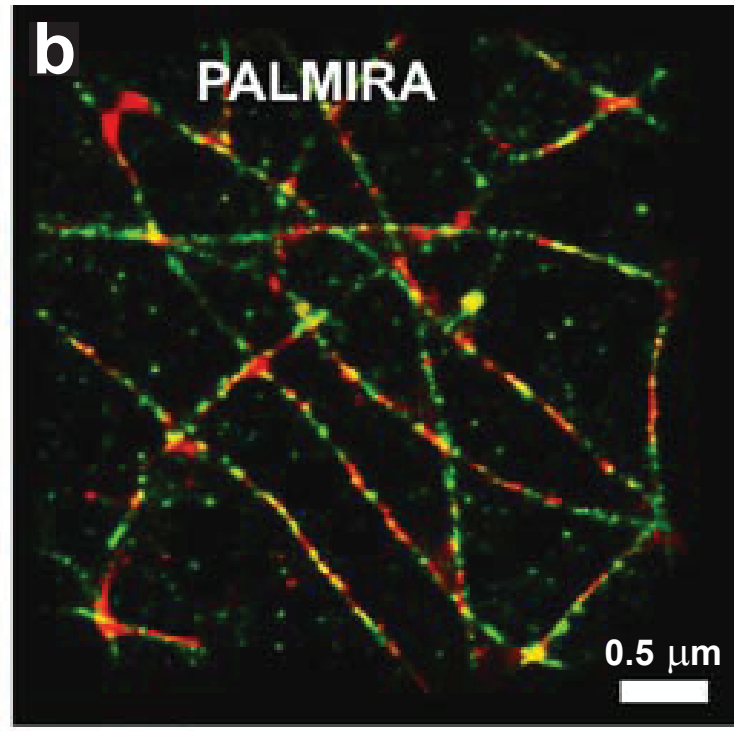

$\max$

Figure 3.3: Two-color images from the microtubular network in a PtK2 cell stained with Cy5 (red) and rsFastLime (green). The super-resolution PALMIRA image is shown in $\mathbf{b}$ and compared to the corresponding conventional wide-field image depicted in a. Images of Cy5 and rsFastLime are constructed from 20000 and 30000 individual CCD frames, respectively, which were recorded in sequential order. The exposure time per frame was $2 \mathrm{~ms}$ and the applied intensities were $I_{633}=10 \mathrm{~kW} / \mathrm{cm}^{2}$ and $I_{488}=4 \mathrm{~kW} / \mathrm{cm}^{2}$. The PALMIRA recordings reveal details about the colocalization of both labels which are not observable in the conventional image. The wide-field image was constructed by adding up the signal from all background-corrected frames; subsequently, it was smoothed using a Lagrangian interpolation of third order.

frame rate of $f=(2 \mathrm{~ms})^{-1}$ was employed for data acquisition. To adjust the mean burst duration to the camera frame rate excitation intensities of $I_{488}=4 \mathrm{~kW} / \mathrm{cm}^{2}$ and $I_{633}=10 \mathrm{~kW} / \mathrm{cm}^{2}$ were chosen. Typically about 200 and 700 photons were recorded from single rsFastLime and Cy5 molecules per on-time cycle, respectively. Given the FWHM of the PSF at the respective emission wavelengths these numbers translate into a localization precision of down to $15 \mathrm{~nm}$ for rsFastLime and $10 \mathrm{~nm}$ for Cy5.

Figure 3.3 shows reconstructed two-color PALMIRA images of the tubular network of a PtK2 cell that was immunostained with both rsFastLime and Cy5 (see appendix A.3 for a detailed staining protocol). For mounting of the cells a solution of $1 \%(w / v)$ PVA in PBS was added. Finally, the sample was spin-coated for 20 s at 5000 revolutions per minute. Embedding of the cells in PVA was necessary to create an environment that allowed efficient dark state shelving of Cy5 (see previous section). A total of 20000 and 30000 CCD images were taken of Cy5 and rsFastLime, respectively, corresponding to acquisition times of 40s and 60s. Thus, the complete two-color image was recorded within 100s. The wide-field image shown in 3.3 a was generated by adding up all background-corrected frames and perform- 
ing a Lagrangian interpolation of third order. The pixel size in the final PALMIRA image $3.3 \mathrm{~b}$ was set to $20 \mathrm{~nm}$. While this value was slightly larger than the expected localization accuracy, it ensured a sufficiently high count rate in each pixel. Due to the exchange of the fluorescence detection filter in-between the recording of the two different dyes, a chromatic shift of $\approx 50-100 \mathrm{~nm}$ was introduced between the Cy5 and the rsFastLime image. This shift was determined by sequentially imaging doubly stained fluorescent beads with the same set of detection filters. Finally, the two images were overlaid by applying the shift to one of the data sets.

When comparing the super-resolution PALMIRA image with its wide-field counterpart the excellent spatial resolution achieved in both detection channels can be observed. Details which remain hidden in the conventional recording are clearly revealed in the super-resolution image. Because both labels were attached to the same cellular structure, the nanoscale colocalization capability of two-color PALMIRA imaging is immediately visible.

It must be mentioned, that before an adequate fraction of Cy5 markers was irreversibly bleached, the concentration of this label was generally too high to allow the recording of single isolated emitters. Also, the afore-described heterogeneities in the blinking behavior of Cy5 could potentially have resulted in non-linearities in the formation of the super-resolution image, as molecules with shorter off-times may have been counted more often than others. Nonetheless, the basic structures of the tubular network were well reproduced in both detection channels.

In summary, two-color far-field fluorescence nanoscopy based on the photoswitching of single emitters was demonstrated for the first time using the reversibly switchable fluorescent protein rsFastLime (emission at 520nm) and the organic dye Cyanine 5 (emission at $670 \mathrm{~nm}$ ). In both cases, the laser light used for fluorescence excitation also served to switch the majority of markers to a dark state. A comparatively low switch-on crosstalk made it possible to image even densely stained samples. Nonetheless, the fraction of simultaneously switched-on markers set an upper limit to the maximum labeling density. The number of detected photons per individual fluorophore allowed to image the tubular network of fixated PtK2 cells at a spatial resolution of $\approx 10-15 \mathrm{~nm}$.

The strategy of recording both detection channels sequentially, as was done here, is by no means mandatory. As long as the wavelength used for photoswitching of one molecular species does not have an unfavorable effect on the photoswitching of the other, both labels can be excited simultaneously and their emission separated by a dichroic mirror in the detection path [52]. It can be anticipated that in the future the use of brighter dyes with improved switching characteristics such as a lower switch-on crosstalk will lead to further advancements in single molecule based fluorescence nanoscopy. Once more, the results presented in this chapter highlight the great potential of molecular photoswitching for far-field light microscopy at the nanoscale. 


\section{The RSFP ASFP595}

The first member of the fluorescent protein family that was reported to feature reversible photoswitching was the red emitting ASFP595 from the sea anemone Anemonia sulcata [67]. The tertiary structure of ASFP595 resembles that of GFP, with its chromophore being embedded in the interior of an 11-stranded $\beta$-can. Like in other FPs, the chromophore is formed autocatalytically from the protein backbone [106]. Light in the green and yellow spectral region $(\approx 530-580 \mathrm{~nm})$ both excites and activates fluorescence peaked at $595 \mathrm{~nm}$, whereas blue irradiation $(\approx 400-460 \mathrm{~nm})$ can be employed to switch the molecule to a nonfluorescent state. Witch a quantum yield of $\approx 1 \%$ ASFP595 is a rather dim emitter. In contrast to the monomeric GFP and rsFastLime, ASFP595 forms tetrameric compounds which limits its suitability as a genetically encodable fluorescent marker as large and heavy markers tend to interfere with the function of the target protein.

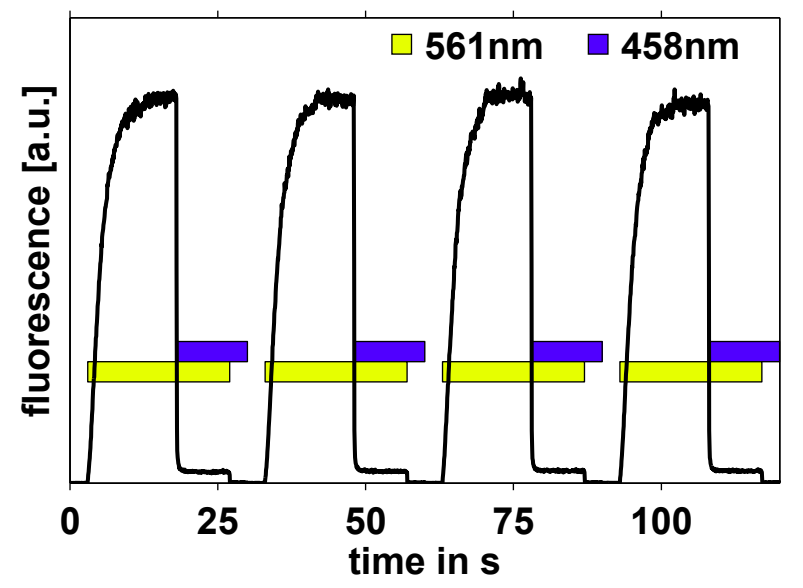

Figure 4.1: Ensemble photoswitching of ASFP595 expressed in E. Coli bacteria. Color bars indicate the timing of the applied illumination at $561 \mathrm{~nm}$ (green/yellow; $I_{561}=5 \mathrm{~W} / \mathrm{cm}^{2}$ ) and $458 \mathrm{~nm}$ (blue; $I_{458}=4.5 \mathrm{~W} / \mathrm{cm}^{2}$ ). $561 \mathrm{~nm}$ light switches molecules to the bright state and elicits fluorescence ('positive switching'). Upon additional irradiation with $458 \mathrm{~nm}$ light fluorescence emission is inhibited with an efficiency of $\approx 97 \%(\approx 3 \%$ residual fluorescence). Importantly, the positive switching of ASFP595 implies that upon simultaneous illumination with yellow and blue light fluorescence emission reaches an equilibrium level which allows arbitrarily long readout times. 

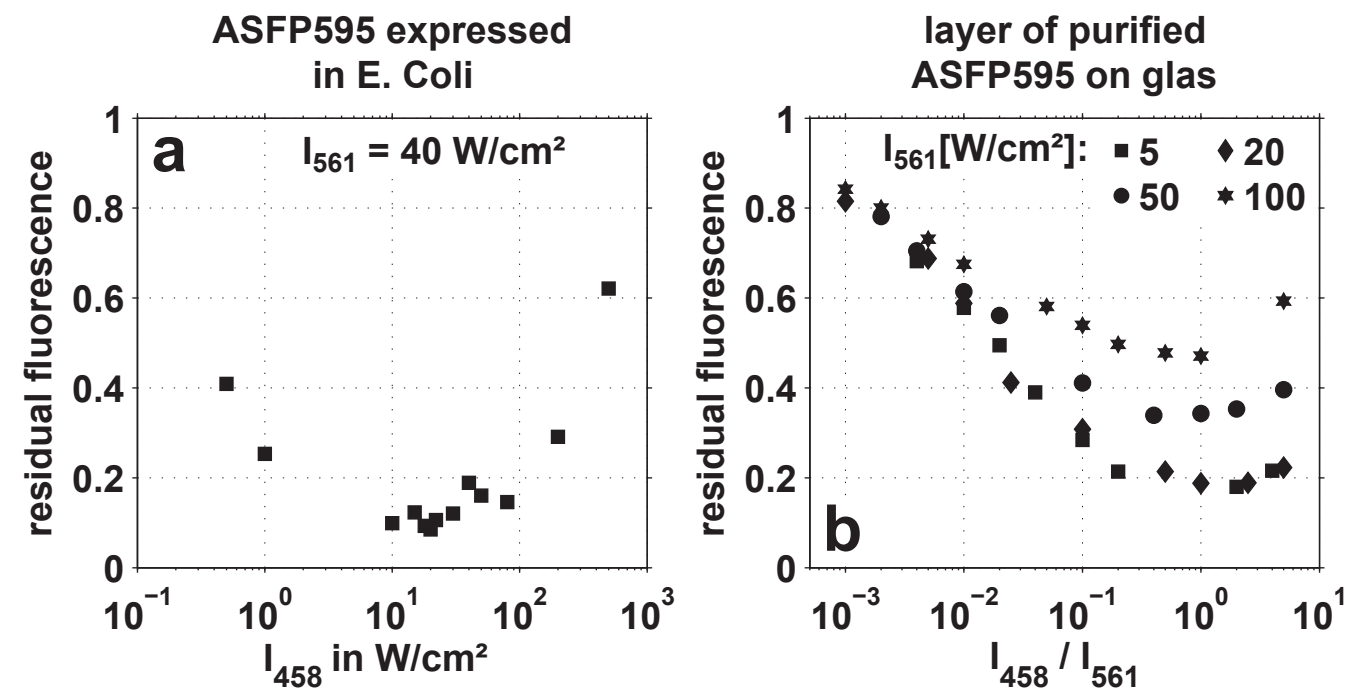

Figure 4.2: Characterization of ASFP595 photoswitching. a ASFP595 expressed in E. coli. For $I_{561}=40$ $\mathrm{W} / \mathrm{cm}^{2}$ and $I_{458} \approx 30 \mathrm{~W} / \mathrm{cm}^{2}$ fluorescence emission is maximally suppressed (residual fluorescence $\approx 10 \%$ ). Increasing $I_{458}$ further leads to a decrease in inhibition efficiency because blue light also elicits fluorescence, effectively limiting the maximum applicable blue intensity. b Purified ASFP595 on a glass surface. Saturation curves recorded for different intensities of the yellow light. Larger values of $I_{561}$ lead to less efficient inhibition of fluorescence.

\subsection{Photoswitching Properties}

Reversible photoswitching of an ensemble of ASFP595 protein expressed in live E. coli bacteria is illustrated in figure 4.1. The experiment was performed on an apparatus similar to the one depicted in figure 2.3 , with two $\mathrm{cw}$ lasers operating at 458nm (blue) and 561nm (yellow) serving as light sources. The timing of the applied illumination is illustrated by the colored bars shown in the lower half of the figure. The intensities used were $I_{561}=5 \mathrm{~W} / \mathrm{cm}^{2}$ and $I_{458}=4.5 \mathrm{~W} / \mathrm{cm}^{2}$.

Visibly, irradiation with yellow light elicits fluorescence and at the same time leads to a gradual increase of the detected signal (positive switching). Upon the onset of simultaneous illumination with blue light fluorescence emission drops to $\approx 3 \%$ of its maximum level. Notably, the emitted signal stays at the $\approx 3 \%$ equilibrium level for as long as both lasers are switched on. In the context of RESOLFT imaging this property of ASFP595 is advantageous as it allows for arbitrarily long fluorescence readout. Remember that the 'negative switching' of rsFastLime (i.e. the fact that the excitation light also switched molecules to the dark state) effectively limits the number of fluorescence photons which can be detected from an individual emitter per switching cycle because longer readout times compromise resolution (see 2.11).

The suitability of ASFP595 as a RESOLFT marker is determined by the reversibility of the switching transition in connection with the degree of saturation that can be realized when switching the protein to the dark state. Figure 4.2 shows saturation curves of ASFP595 
expressed in live E. coli bacteria (a) and of the purified protein suspended in a PBS buffer solution and adsorbed on a microscope cover glass (b). The illumination scheme followed that already illustrated in figure 4.1. ASFP595 was maximally switched on via irradiation with yellow light; subsequently, simultaneous illumination with blue light lead to a decrease in fluorescence until an equilibrium was reached. The 'residual fluorescence' depicted in figure 4.2 denotes the equilibrium level reached after switch-off in multiples of the maximum signal.

The data reveal the two main restrictions limiting the use of ASFP595 as a switchable label in super-resolution RESOLFT imaging. Figure 4.2 a clearly shows that while blue light can be successfully employed to inhibit fluorescence emission, the switch-off efficiency deteriorates for larger values of $I_{458}$. This finding has been attributed to a non-zero excitation crosstalk of the $458 \mathrm{~nm}$ light, meaning that at high intensities the blue inhibition light itself elicits fluorescence [68, 69]. Consequently, the switch-off intensity cannot be arbitrarily enhanced which effectively limits the maximum attainable resolution.

Secondly, it can be seen from figure $4.2 \mathrm{~b}$ that an increase in the excitation intensity is accompanied by a reduction of the switch-off efficiency. This means that in order to achieve an adequate signal to noise ratio in the RESOLFT image small activation and excitation intensities have to be used which in turn comes at the cost of prolonged switching times. Indeed, this characteristic of ASFP595 calls for a compromise between accelerated image acquisition on one hand and switch-off efficiency on the other. According to a photophysical model of ASFP595 developed in [69], the increase in the maximum achievable residual fluorescence level for higher values of $I_{561}$ can be ascribed to the existence of a second bright state of the chromophore. Specifically, it has been suggested that this second fluorescent state cannot be quenched by blue light and is increasingly populated at high yellow intensities. Despite these restraints it must be pointed out that at an excitation intensity of $I_{561} \approx 10 \mathrm{~W} / \mathrm{cm}^{2}$ the saturation intensity for the inhibition of fluorescence with $458 \mathrm{~nm}$ light is as low as $\approx 100 \mathrm{~mW} / \mathrm{cm}^{2}$. This constitutes a decrease by factors of $\approx 10^{7}$ and $\approx 10^{4}$ as compared to STED and GSD microscopy, respectively.

\subsection{Super-Resolution RESOLFT Imaging}

Earlier, ASFP595 was successfully employed as a RESOLFT marker in a point-scanning setup [68]. By overlaying a diffraction-limited 568nm excitation spot with a doughnutshaped 458nm inhibition beam a sample of ASFP595-stained nanofabricated grooves was imaged at less than $100 \mathrm{~nm}$ resolution. This demonstration marked the first use of protein photoswitching in far-field fluorescence nanoscopy. However, while these experiments exemplified the potential of ASFP595 and of molecular photoswitching for subdiffraction microscopy, the data acquisition process remained slow.

In this work, RESOLFT imaging with ASFP595 was realized using a parallelized wide-field setup similar to the one depicted in figure 2.12, which allowed for significantly faster data acquisition [14, 15, 74]. Note that parallelization of the imaging process is rendered possible because saturating the switch-off transition in ASFP595 can be accomplished already at very low light intensities $\left(I_{\text {sat }} \approx 1 \mathrm{~W} / \mathrm{cm}^{2}\right)$. A homogeneous spot of $561 \mathrm{~nm}$ excitation/activation 


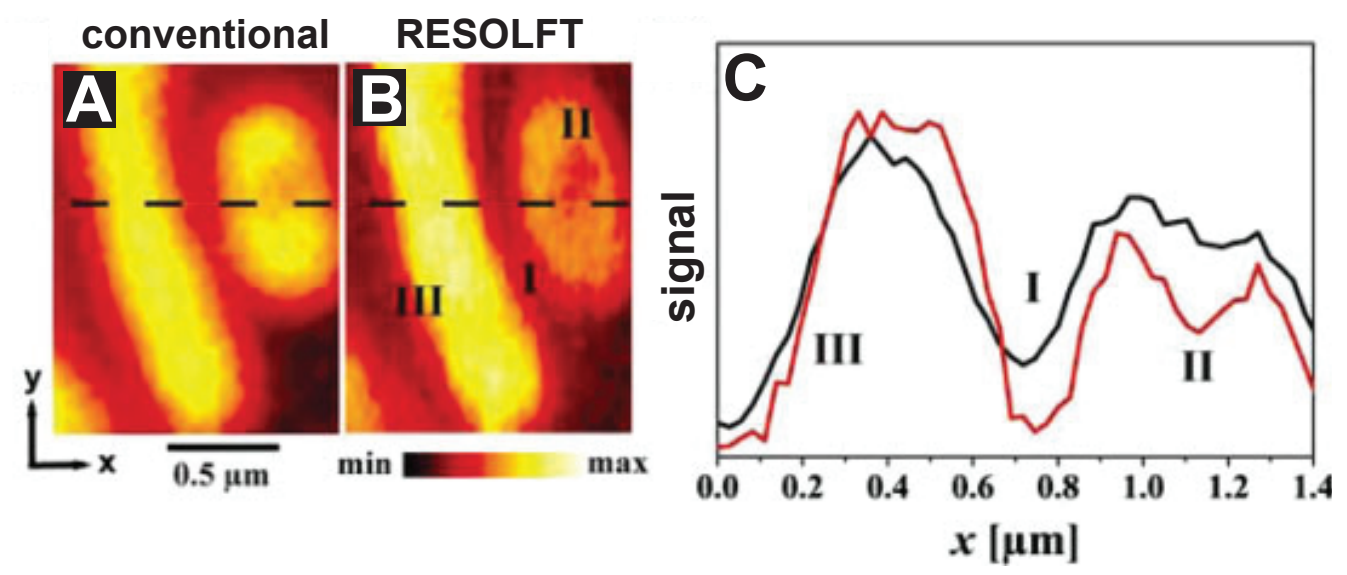

Figure 4.3: Wide-field images of live E. coli bacteria expressing ASFP595 without (A) and with (B) patterned illumination by blue switch-off light. The modulated inhibition pattern was formed along the lateral x-direction with a periodicity $D=500 \mathrm{~nm}$. The intensities used were $I_{561}=5 \mathrm{~W} / \mathrm{cm}^{2}$ and $I_{458}=4.5 \mathrm{~W} / \mathrm{cm}^{2}$. C Normalized line profile along the $\mathrm{x}$-direction corresponding to the dashed line in $\mathbf{A}$ and $\mathbf{B}$ (black conventional and red RESOLFT image). In the RESOLFT image (B) further details are resolved along the patterned x-axis (I, II and III).

light (diameter $20 \mu \mathrm{m}$ ) was superimposed with an equally sized stripe-like $458 \mathrm{~nm}$ inhibition pattern along the $\mathrm{x}$-direction (distance between neighboring maxima $D=500 \mathrm{~nm}$ ). The structured switch-off profile was scanned over the sample through a full period $D$ in 20 equidistant steps, resulting in a stepsize of $25 \mathrm{~nm}$. Throughout the image acquisition process the sample was continuously subjected to homogeneous illumination at $561 \mathrm{~nm}\left(I_{561}=5 \mathrm{~W} / \mathrm{cm}^{2}\right)$ and simultaneous irradiation with the $458 \mathrm{~nm}$ inhibition pattern (peak intensity: $I_{458}=4.5 \mathrm{~W} / \mathrm{cm}^{2}$ ). The intensity values were chosen to enable efficient fluorescence inhibition and a sufficiently large degree of saturation of the switch-off transition (here $\xi:=I_{458} / I_{\text {sat }} \approx 30$ ). The use of higher inhibition intensities was compromised by excitation cross talk of the blue light and by optical imperfections in the form of incomplete intensity-zeros of the $458 \mathrm{~nm}$ inhibition light pattern (see figure 2.15). With the parameters employed here an optical resolution of $\approx 50 \mathrm{~nm}$ could be anticipated [74].

During each scanning step, fluorescence emission was allowed to equilibrate for $15 \mathrm{~s}$ to ensure that previously switched-off ASFP595 molecules now located in the minima of the inhibition pattern were maximally switched to the fluorescent state (see figure 4.1). For the last $0.5 \mathrm{~s}$ the $\mathrm{CCD}$ was exposed. Thus, the image acquisition time amounted to a total of $300 \mathrm{~s}$. Due to the saturated switch-off with the modulated $458 \mathrm{~nm}$ inhibition light fluorescence emission was restricted to a subdiffraction-sized vicinity of the blue intensity zeros. Reconstruction of the final RESOLFT-image was performed as outlined earlier (see figure 2.16). In order to account for the offset formed by incomplete depletion, $3 \%$ of the original wide-field image was subtracted from each striped image, as discussed earlier (see equation 2.7).

Figure 4.3 shows a conventional wide-field image of live E. coli bacteria expressing ASFP595 (A) along with its super-resolution RESOLFT counterpart (B). The latter shows details along 
the $\mathrm{x}$-direction which are not disclosed in the conventional wide-field image. As outlined by the $\mathrm{x}$-line scan displayed in figure $4.3(\mathbf{C})$, neighboring bacteria are better separated (I), structures inside the bacteria better revealed (II), and the edges of the bacteria sharpened (III). To rule out artifacts in the image reconstruction process, similar profiles along the y-direction of both conventional and RESOLFT recording were compared and found to be congruent in all cases (data not shown). This was the expected result as the super-resolution recording scheme adopted here did not provide resolution enhancement along the y-direction. 


\section{Conclusion}

Far-field fluorescence microscopy is one of the most powerful and versatile tools in the live sciences. By virtue of its high specificity, non-invasiveness and 3D imaging capability it enables the study of the structure and function of living organisms on the cellular and subcellular level. In the recent decade it was shown that the final limitation of this technique, namely its limited resolving power, could be overcome by photoswitching the fluorescent labels between a dark and a bright state. The rationale common to all far-field fluorescence nanoscopy methodologies is to make use of molecular photoswitching to single out and record bright structures of subdiffraction size sequentially in time. In this context the use of reversibly switchable fluorescenct proteins (RSFPs) as marker molecules is of particular interest as they can be expressed inside living cells and may be fused to target proteins and organelles while causing only minimal perturbations of native cell functions.

In this thesis it was demonstrated for the first time that the same molecular switching mechanism, namely the cis-trans isomerization of the chromophore of the RSFP rsFastLime, could be employed for both ensemble- and single molecule based super-resolution microscopy. Hence, the results presented in this work furnish unequivocal proof that both techniques are grounded on the same basic principle.

The longevity of the dark state of rsFastLime enabled RESOLFT imaging at switchoff intensities as low as $\approx \mathrm{W} / \mathrm{cm}^{2}$, keeping photo stress and irradiation induced damage of the specimen to a minimum and allowing for parallelized camera-based image acquisition. Related techniques such as GSD or STED require much higher switch-off intensities (GSD $\approx 10^{3} \mathrm{~W} / \mathrm{cm}^{2}$, STED $\approx 10^{9} \mathrm{~W} / \mathrm{cm}^{2}$ ), because the transitions competing with the light induced fluorescence inhibition process occur on much faster time scales. Also, the low light intensities and comparatively long illumination times $(\approx$ ms-s) used in RESOLFT microscopy based on protein photoswitching could be realized using $\mathrm{cw}$ lasers and simple optical components like acousto optical filters and mechanical shutters. It can be envisioned that in the future similar microscopes may be powered by an ordinary low-power and low-cost fluorescence lamp.

It could be confirmed that resolution in RESOLFT microscopy with rsFastLime is no longer limited by diffraction. As an application, the endoplasmatic reticulum of fixated PtK2 cells was imaged at a resolution of $<100 \mathrm{~nm}$ along one lateral direction. However, it must be noted that super-resolution imaging with rsFastLime is by no means restricted to one dimension. The 1D setup employed in this work was mainly chosen for ease of use and could easily be extended to $2 \mathrm{D}$.

Practically, resolution in RESOLFT microscopy using rsFastLime was limited by the insufficient photostability of the marker. Since the technique relies on the generation of subdiffrac- 
tion sized focal volumes with diffraction limited beams an individual molecule is switched on and -off multiple times before its fluorescence contributes to the final image. E.g., enhancing resolution by a factor of two involves increasing the switch-off intensity by four-fold and the number of on-off cycles per marker molecule by a factor of two for each scanning direction (Niquist criterion).

The fact that the same light which generates the fluorescence signal also switches molecules to the dark state ('negative switching') complicates RESOLFT-imaging with rsFastLime, particularly in connection with the protein's low photostability. As a result of the 'negative switching' property, the number of photons which can be detected per molecule and per switching cycle (off-on-off) is restricted; additionally, longer fluorescence readout comes at the expense of an impaired resolution. These difficulties do not arise when using 'positive' switches such as ASFP595, as markers of this sort allow arbitrarily long readout times without compromising resolution.

It can be anticipated that in the future the aforementioned hurdles, namely the limited photostability of rsFastLime and its 'negative switching' mode, can be overcome. Specifically, one of the great advantages of fluorescent proteins lies in the possibility to modify their fluorescence- and switching properties by targeted mutagenesis. E.g., just recently the generation of a 'positive switching' mutant of the RSFP Dronpa was reported [97]. The ideal RSFP for RESOLFT imaging should be monomeric to facilitate fusion to target proteins and feature fast 'positive switching', efficient switch-off, little to no switching fatigue and high brightness. The great prospect of combining the advantages of highly specific and noninvasive in vivo labeling with an imaging technique that provides nanoscale resolution at ultra-low light intensities while being purely physical in nature (i.e. it requires no image processing) makes the search for RSFPs with improved photophysical properties a highly promising subject of future research.

An important result of this work consisted in the demonstration that the same molecular switching mechanism which enabled RESOLFT microscopy with rsFastLime could be employed to realize single-molecule based super-resolution imaging. Specifically, The relatively low switch-on cross talk of the $491 \mathrm{~nm}$ excitation light made it possible to implement SMS microscopy with rsFastLime using only a single laser. By adapting the duration of a single-molecule photon burst to the frame time of the freely running CCD camera, singlemolecule events could be recorded at a maximum signal to noise ratio. The number of $\approx 200$ detected photons per rsFastLime molecule allowed the imaging of the microtubular network of fixated PtK2 cells at a resolution of $\approx 20 \mathrm{~nm}$ within a recording time of $60 \mathrm{~s}$. Further, the technique was extended to two-color imaging by sequentially reading out the fluorescence signal of two spectrally distinct switchable markers. Since in SMS microscopy the subdiffraction 'foci' are created by stochastically switching-on single emitters, an individual marker molecule has to be switched on and -off only once - a property which marks an important difference between ensemble- and single molecule based far-field fluorescence nanoscopy techniques. As a consequence, markers such as rsFastLime for which switching fatigue constitutes a major challenge in RESOLFT microscopy may serve as viable labels in single-molecule based super-resolution imaging. Nonetheless, improved photostability is 
desirable also in SMS microscopy because the repeated off-on-off blinking of an individual emitter serves to enhance image contrast.

While the switch-on crosstalk of the excitation light simplifies SMS data acquisition of rsFastLime molecules by rendering a second UV switch-on laser optional, this crosstalk imposes an upper bound on the maximum feasible labeling density. Higher concentrations may cause the images of nearby molecules to overlap on the detector, thus compromising localization accuracy. Although it is possible to reduce the initial marker concentration by bleaching some of the emitters prior to the actual data acquisition, this procedure is likely to induce artifacts as the photobleaching rate may vary throughout the sample (e.g. depending on local conditions such as $\mathrm{pH}$ or the concentration of molecular oxygen). In order to avoid these limitations it is of great importance to find markers which feature a significantly lower switch-on cross talk of the excitation light.

A general problem in super-resolution SMS microscopy is the suppression of single-molecule events of low brightness. While imposing a lower threshold on the number of photons per burst cannot be avoided if light emitting markers are to be discerned from diffuse background, this procedure leads to an effective detection efficiency which scales non-linearly with molecular brightness. As a result, the final image may not reflect the 'true' distribution of fluorescence labels in all detail.

In summary, the diffraction barrier in far-field fluorescence microscopy was overcome by fluorescent protein photoswitching. In particular, using the same marker molecule for both ensemble- and single-molecule based super-resolution imaging underscored that it is the same photoswitching mechanism which lies at the heart of both methods. Nonetheless, the particular characteristics of the reversibly switchable fluorescent protein used in these studies made it an imperfect label for both techniques, leaving ample space for improvement of its photophysical properties such as photostability and brightness. It can be expected that the discovery of new switchable fluorescent probes and the refinement of existing ones will substantially enhance the power of far-field fluorescence nanoscopy. Ultimately, these technologies will help to further unravel the functioning of the cellular machinery at the nanoscale. 


\section{Bibliography}

[1] E. Abbe. Beiträge zur theorie des mikroskops und der mikroskopischen wahrnehmung. Arch. Mikr. Anat., 9:413-468, 1873. 7,9

[2] A.I. Kirkland and L Hutchinsons. Atomic resolution transmission electron microscopy. In P.W. Hawkes and J.C. Spence, editors, Science of Microscopy, volume 1, page 3-64. Springer, 2007. 7

[3] G. Binnig and H. Rohrer. Scanning tunneling microscopy. Helv. Phys. Acta, 55:726735, 1982.7

[4] G. Binnig, C.F. Quate, and Ch. Gerber. Atomic force microscope. Phys. Rev. Lett., 56:930-933, 1986. 7

[5] S.W. Hell. Far-field optical nanoscopy. Science, 316(5828):1153-1158, 2007. 8, 15 , $16,18,63,64,67$

[6] M. Minsky. Microscopy apparatus, us patent 3,013,467, 1961. 10

[7] D. Magde, E. L. Elson, and W. W. Webb. Thermodynamic fluctuations in a reacting system - measurement by fluorescence correlation spectroscopy. Phys. Rev. Lett., 29(11):705-708, 1972. 10

[8] S. W. Hell and E. H. K. Stelzer. Fundamental improvement of resolution with a 4pi-confocal fluorescence microscope using two-photon excitation. Opt. Commun., 93:277-282, 1992. 10

[9] H. Gugel, J. Bewersdorf, S. Jakobs, J. Engelhardt, R. Storz, and S.W. Hell. Cooperative 4pi excitation and detection yields 7-fold sharper optical sections in live cell microscopy. Biophys. J., 87:4146-4152, 2004. 10

[10] M.G.L. Gustafsson, D.A. Agard, and J.W. Sedat. I5m: 3d widefield light microscopy with better than $100 \mathrm{~nm}$ axial resolution. J. Microsc., 195:10-16, 1999. 10

[11] W. Denk, J. H. Strickler, and W. W. Webb. Two-photon laser scanning fluorescence microscopy. Science, 248:73-76, 1990. 10

[12] D.W. Pohl. Near-field optics: comeback of light in microscopy. Solid State Phenom., 63-64:252-256, 1998. 11

[13] S. W. Hell and J. Wichmann. Breaking the diffraction resolution limit by stimulated emission: stimulated emission depletion microscopy. Opt. Lett., 19(11):780-782, 1994. 11, 15,43 
[14] S.W. Hell. Toward fluorescence nanoscopy. Nat. Biotechnol., 21(11):1347-1355, 2003. 12, 22, 64, 65, 77

[15] S.W. Hell, S. Jakobs, and L. Kastrup. Imaging and writing at the nanoscale with focused visible light through saturable optical transitions. Appl. Phys. A, 77:859-860, 2003. 12, 15,77

[16] S.W. Hell. Strategy for far-field optical imaging and writing without diffraction limit. Phys. Lett. A, 326(1-2):140-145, 2004. 15

[17] R. Menon and H.I. Smith. Absorbance-modulation optical lithography. J. Opt. Soc. Am. A, 23(9):2290-2294, 2006. 15

[18] R. Menon, H. Y. Tsai, and S. W. Thomas. Far-field generation of localized light fields using absorbance modulation. Phys. Rev. Lett., 98(4), 2007. 15

[19] T. A. Klar, S. Jakobs, M. Dyba, A. Egner, and S. W. Hell. Fluorescence microscopy with diffraction resolution limit broken by stimulated emission. Proc. Nat. Acad. Sci. U.S.A., 97:8206-8210, 2000. 15, 43

[20] G. Donnert, J. Keller, R. Medda, M. A. Andrei, S. O. Rizzoli, R. Lührmann, R. Jahn, C. Eggeling, and S. W. Hell. Macromolecular-scale resolution in biological fluorescence microscopy. Proc. Nat. Acad. Sci. U.S.A., 103(31):11440-11445, 2006. 15

[21] R. Schmidt, C. A. Wurm, S. Jakobs, J. Engelhardt, A. Egner, and S. W. Hell. Spherical nanosized spot unravel the interior of cells. Nat. Meth., 4(1):81-86, 2008. 15

[22] V. Westphal and S.W. Hell. Nanoscale resolution in the focal plane of an optical microscope. Phys. Rev. Lett., 94:143903, 2005. 15

[23] V. Westphal, S. O. Rizzoli, M. A. Lauterbach, D. Kamin, R. Jahn, and S. W. Hell. Video-rate far-field optical nanoscopy dissects synaptic vesicle movement. Science, 320:246-249, 2008. 15, 67

[24] K. I. Willig, S. O. Rizzoli, V. Westphal, R. Jahn, and S. W. Hell. Sted-microscopy reveals that synaptotagmin remains clustered after synaptic vesicle exocytosis. Nature, 440(7086):935-939, 2006. 15

[25] R. J. Kittel, C. Wichmann, T. M. Rasse, W. Fouquet, M. Schmidt, A. Schmid, D. A. Wagh, C. Pawlu, R. Kellner, K. I. Willig, S. W. Hell, E. Buchner, M. Heckmann, and S. J. Sigrist. Bruchpilot promotes active zone assembly, ca2+-channel clustering, and vesicle release. Science, 312:1051-1054, 2006. 15

[26] L. Kastrup, H. Blom, C. Eggeling, and S.W. Hell. Fluorescence fluctuation spectroscopy in subdiffraction focal volumes. Phys. Rev. Lett., 94:178104, 2005. 15

[27] S. W. Hell and M. Kroug. Ground-state depletion fluorescence microscopy, a concept for breaking the diffraction resolution limit. Appl. Phys. B, 60:495-497, 1995. 15, 17 , 23,39 
[28] S. Bretschneider, C. Eggeling, and S. W. Hell. Breaking the diffraction barrier in fluorescence microscopy by optical shelving. Phys. Rev. Lett., 98:218103, 2007. 16. 17, 23, 39.

[29] W. Heisenberg. The physical principles of the quantum theory. Chicago Univ Press, Chicago, 1930. 16, 55

[30] X. Qu, D. Wu, L. Mets, and N.F. Scherer. Nanometer-localized multiple singlemolecule fluorescence microscopy. Proc. Nat. Acad. Sci. U.S.A., 101(31):1129811303, 2004. 17

[31] L. S. Churchman, Z. Okten, R. S. Rock, J. F. Dawson, and J. A. Spudich. Single molecule high-resolution colocalization of cy 3 and cy5 attached to macromolecules measures intramolecular distances through time. Proc. Nat. Acad. Sci. U.S.A., 102(5):1419-1423, 2005. 17

[32] A. Yildiz, J. N. Forkey, S. A. McKinney, T. Ha, Y. E. Goldman, and P. R. Selvin. Myosin v walks hand-over-hand: single fluorophore imaging with 1.5-nm localization. Science, 300(5628):2061-5, 2003. 17

[33] M.K. Cheezum, W.F. Walker, and W.H. Guilford. Quantitative comparison of algorithms for tracking single fluorescent particles. Biophys. J., 81:2378-2388, 2001. 17

[34] R.E. Thompson, D.R. Larson, and W.W. Webb. Precise nanometer localization analysis for individual fluorescent probes. Biophys. J., 82:2775-2783, 2002. 17, 57, 58

[35] E. Betzig, G.H. Patterson, R. Sougrat, O.W. Lindwasser, S. Olenych, J.S. Bonifacino, M.W. Davidson, J. Lippincott-Schwartz, and H.F. Hess. Imaging intracellular fluorescent proteins at nanometer resolution. Science, 313(5793):1642-1645, 2006. 17, 55, 66

[36] G.H. Patterson and J. Lippincott-Schwartz. A photoactivatable gfp for selective photolabeling of proteins and cells. Science, 297(5588):1873-1877, 2002. 17, 55

[37] R. Ando, H. Hama, M. Yamamoto-Hino, H. Mizuno, and A. Miyawaki. An optical marker based on the uv-induced green-to-red photoconversion of a fluorescent protein. Proc. Nat. Acad. Sci. U.S.A., 99(20):12651-12656, 2002. 17

[38] R. Ando, H. Mizuno, and A. Miyawaki. Regulated fast nucleocytoplasmic shuttling observed by reversible protein highlighting. Science, 306(5700):1370-1373, 2004. 17, 18,47

[39] J. Wiedenmann, S. Ivanchenko, F. Oswald, F. Schmitt, C. Röcker, A. Salih, K.-D. Spindler, and G.U. Nienhaus. Eosfp, a fluorescent marker protein with uv-inducible green-to-red fluorescence conversion. Proc. Nat. Acad. Sci. U.S.A., 101(45):1590515910, 2004. 17, 55 
[40] K. A. Lukyanov, D. M. Chudakov, S. Lukyanov, and V. V. Verkhusha. Photoactivatable fluorescent proteins. Nat. Rev. Mol. Cell Biol., 6:885-891, 2005. 17, 18, 55

[41] D. Axelrod. Total internal-reflection fluorescence microscopy. Methods in Cell Biology, 30:245-270, 1989. 17

[42] S.T. Hess, T. P. K. Girirajan, and M.D. Mason. Ultra-high resolution imaging by fluorescence photoactivation localization microscopy. Biophys. J., 91(11):4258-4272, 2006. 17, 55, 66, 67

[43] M.J. Rust, M. Bates, and X.W. Zhuang. Sub-diffraction-limit imaging by stochastic optical reconstruction microscopy (storm). Nat. Meth., 3:793-796, 2006. 17, 55, 66

[44] T. Ha and J. Xu. Photodestruction intermediates probed by an adjacent reporter molecule. Phys. Rev. Lett., 90(22), 2003. 17

[45] M. Bates, T.R. Blosser, and X. Zhuang. Short-range spectroscopic ruler based on a single-molecule optical switch. Phys. Rev. Lett., 94:108101, 2005. 17, 55, 70, 71,

[46] M. Heilemann, E. Margeat, R. Kasper, M. Sauer, and P. Tinnefeld. Carbocyanine dyes as efficient reversible single-molecule optical switch. J. Am. Chem. Soc., 127(11):3801-3806, 2005. 17, 55, 70, 71,

[47] C. Geisler, A. Schönle, C. von Middendorff, H. Bock, C. Eggeling, A. Egner, and S. W. Hell. Resolution of 1/10 in fluorescence microscopy using fast single molecule photo-switching. Appl. Phys. A, 88(2):223-226, 2007. 17, 57, 66

[48] A. Egner, C. Geisler, C. von Middendorff, H. Bock, D. Wenzel, R. Medda, M. Andresen, A.C. Stiel, S. Jakobs, C. Eggeling, A. Schönle, and S.W. Hell. Fluorescence nanoscopy in whole cells by asnychronous localization of photoswitching emitters. Biophys. J., 93:3285-3290, 2007. 17, 57, 66

[49] H. Bock, C. Geisler, C. A. Wurm, S. Jakobs, A. Schönle, A. Egner, S. W. Hell, and C. Eggeling. Two-color far-field fluorescence nanoscopy based on photoswitching emitters. Appl. Phys. B, 88(161-165), 2007. 17, 68

[50] H. Shroff, C. G. Galbraith, J. A. Galbraith, H. White, J. Gillette, S. Olenych, M. W. Davidson, and E. Betzig. Dual-color superresolution imaging of genetically expressed probes within individual adhesion complexes. Proc. Nat. Acad. Sci. U.S.A., 104(51):20308-20313, 2007. 17, 68

[51] M. Bates, B. Huang, G.P. Dempsey, and X. Zhuang. Multicolor super-resolution imaging with photo-switchable fluorescent probes. Science, 317:1749-1753, 2007. 17, 68

[52] M.L. Bossi, J. Fölling, V.N. Belov, V.P. Boyarskiy, R. Medda, A. Egner, C. Eggeling, A. Schönle, and S.W. Hell. Multi-color far-field fluorescence nanoscopy through isolated detection of distinct molecular species. Nano Lett., 8:2463-2468, 2008. 17, 68, 74 
[53] J. Fölling, V. Belov, R. Kunetsky, R. Medda, A. Schönle, A. Egner, C. Eggeling, M. Bossi, and S. W. Hell. Photochromic rhodamines provide nanoscopy with optical sectioning. Angew. Chem. Int. Ed., 46:6266-6270, 2007. 17, 66

[54] B. Huang, W. Wang, M. Bates, and X. Zhuang. Three-dimensional super-resolution imaging by stochastic optical reconstruction microscopy. Science, 319:810-813, 2008. 17

[55] M.F. Juette, T.J. Gould, M.D. Lessard, M.J. Mlodzianoski, B.S. Nagpure, B.T. Bennett, S.T. Hess, and J. Bewersdorf. Three-dimensional sub-100 nm resolution fluorescence microscopy of thick samples. Nat. Meth., 5(6):527-529, 2008. 17

[56] H. Shroff, C.G. Galbraith, J.A. Galbraith, and E. Betzig. Live-cell photoactivated localization microscopy of nanoscale adhesion dynamics. Nat. Meth., 5(5):417-423, 2008. 17

[57] A. C. Stiel, M. Andresen, H. Bock, M. Hilbert, J. Schilde, A. Schönle, C. Eggeling, A. Egner, S. W. Hell, and S. Jakobs. Generation of monomeric reversibly switchable red fluorescent proteins for far-field fluorescence nanoscopy. Biophys. J., 95:2989-2997, 2008. 17

[58] J. Fölling, M. Bossi, H. Bock, R. Medda, C. A. Wurm, B. Hein, S. Jakobs, C. Eggeling, and S. W. Hell. Fluorescence nanoscopy by ground-state depletion and singlemolecule return. Nat. Meth., 2008. 17,68

[59] T. Shimomura, F.H. Johnson, and Y. Saiga. Extraction, purification and properties of aequorin, a bioluminescent protein from the luminous hydromedusan, aequorea. $J$. Cell. Comp. Physiol., 59:223-240, 1962. 18

[60] D. Prasher, V.K. Eckenrode, W.W. Ward, F.G. Prendergast, and Cormier M.J. Primary structure of the aequorea victoria green-fluorescent protein. Gene, 111:229-233, 1992. 18

[61] M. Chalfie, Y. Tu, G. Euskirchen, W. W. Ward, and D. C. Prasher. Green fluorescent protein as a marker for gene expression. Science, 263:802-804, 1994. 18

[62] Roger Y. Tsien. The green fluorescent protein. Annu. Rev. Biochem., 67:509-544, 1998. 18,63

[63] R. Heim, D.C. Prasher, and R.Y. Tsien. Wavelength mutations and posttranslational autoxidation of green fluorescent protein. Proc. Nat. Acad. Sci. U.S.A., 91:1250112504, 1994. 18

[64] Y.A. Labas, N.G. Gurskaya, Y.G. Yanushevich, A.F. Fradkov, K.A. Lukyanov, S.A. Lukyanov, and M.V. Matz. Diversity and evolution of the green fluorescent protein family. Proc. Nat. Acad. Sci. U.S.A., 99(7):4256-4261, 2002. 18 
[65] N. C. Shaner, R. E. Campbell, P. A. Steinbach, B. N. Giepmans, A. E. Palmer, and R. Y. Tsien. Improved monomeric red, orange and yellow fluorescent proteins derived from discosoma sp. red fluorescent protein. Nat. Biotechnol., 22:1567-1572, 2004. 18

[66] J. Lippincott-Schwartz, N. Altan-Bonnet, and G. H. Patterson. Photobleaching and photoactivation: following protein dynamics in living cells. Nat. Cell Biol., pages S7-S14, 2003. 18

[67] Konstantin A. Lukyanov, Arkady F. Fradkov, Nadya G. Gurskaya, Mikhail V. Matz, Yulii A. Labas, Aleksandr P. Savitsky, Mikhail L. Markelov, Andrey G. Zaraisky, Xiaoning Zhao, Yu Fang, Wenyan Tan, and Sergey A. Lukyanov. Natural animal coloration can be determined by a nonfluorescent green fluorescent protein homolog. J. Biol. Chem., 275(34):25879-25882, 2000. 18,75

[68] M. Hofmann, C. Eggeling, S. Jakobs, and S.W. Hell. Breaking the diffraction barrier in fluorescence microscopy at low light intensities by using reversibly photoswitchable proteins. Proc. Natl. Acad. Sci. U.S.A., 102(49):17565-17569, 2005. 18, 23, 39 , 44, 64, 65, 77

[69] M. Hofmann. RESOLFT Mikroskopie mit photoschaltbaren Proteinen. PhD thesis, Ruprecht-Karls-Universität Heidelberg, 2007. 19, 77, 93

[70] S. Habuchi, R. Ando, P. Dedecker, W. Verheijen, H. Mizuno, A. Miyawaki, and J. Hofkens. Reversible single-molecule photoswitching in the gfp-like fluorescent protein dronpa. Proc. Nat. Acad. Sci. U.S.A., 102(27):9511-9516, 2005. 19, 25, 47 , 53, 66

[71] P.G. Wilmann, .K Turcic, J.M. Battad, M.C.J. Wilce, R.J. Devenish, M. Prescott, and J. Rossjohn. The 1.7 angstrom crystal structure of dronpa: A photoswitchable green fluorescent protein. J. Mol. Biol., 364:213-224, 2006. 19

[72] A. C. Stiel, S. Trowitzsch, G. Weber, M. Andresen, C. Eggeling, S. W. Hell, S. Jakobs, and M. C. Wahl. 1.8 a bright-state structure of the reversibly switchable fluorescent protein dronpa guides the generation of fast switching variants. Biochem. J., 402(1):35-42, 2007. 19, 20, 26, 63, 68, 70

[73] M.A. Schwentker. Parallelized Ground State Depletion. PhD thesis, Ruprecht-KarlsUniversität Heidelberg, 2007. 27

[74] M. Schwentker, H. Bock, M. Hofmann, S. Jakobs, J. Bewersdorf, C. Eggeling, and S. W. Hell. Wide-field subdiffraction resolft microscopy using fluorescent protein photoswitching. Micr. Res. Tech., 70(3):269-280, 2007. 39, 64, 65, 77, 78

[75] W. Lukosz and M. Marchand. Verfahren zur optischen abbildung unter Überschreitung der beugungsbedingten auflösungsgrenze. Opt. Acta, 10:241-255, 1963. 43 
[76] R. Heintzmann and C. Cremer. Laterally modulated excitation microscopy: improvement of resolution by using a diffraction grating. SPIE Proc., 3568:185-195, 1999. 43,65

[77] M. G. L. Gustafsson. Surpassing the lateral resolution limit by a factor of two using structured illumination microscopy. J. Microsc., 198(2):82-87, 2000. 43, 65

[78] J. T. Frohn, H. F. Knapp, and A. Stemmer. True optical resolution beyond the rayleigh limit achieved by standing wave illumination. Proc. Nat. Acad. Sci. U.S.A., 97:72327236, 2000. 43

[79] R. Heintzmann, T.M. Jovin, and C. Cremer. Saturated patterned excitation microscopy - a concept for optical resolution improvement. J. Opt. Soc. Am. A, 19(8):1599-1609, 2002. 43, 65

[80] M. G. L. Gustafsson. Nonlinear structured-illumination microscopy: Wide-field fluorescence imaging with theoretically unlimited resolution. Proc. Nat. Acad. Sci. U.S.A., 102(37):13081-13086, 2005. 43, 44, 65

[81] L. Shao, B. Isaac, S. Uzawa, D. A. Agard, J. W. Sedat, and M. G. L. Gustafsson. (is)s-5: Wide-field light microscopy with 100-nm-scale resolution in three dimensions. Biophys. J., 94(12):4971-4983, 2008. 43, 65

[82] M. G. L. Gustafsson, L. Shao, P. M. Carlton, C. J. R. Wang, I. N. Golubovskaya, W. Z. Cande, D. A. Agard, and J. W. Sedat. Three-dimensional resolution doubling in widefield fluorescence microscopy by structured illumination. Biophys. J., 94(12):49574970, 2008. 43, 65

[83] L. Schermelleh, P. M. Carlton, S. Haase, L. Shao, L. Winoto, P. Kner, B. Burke, M. C. Cardoso, D. A. Agard, M. G. L. Gustafsson, H. Leonhardt, and J. W. Sedat. Subdiffraction multicolor imaging of the nuclear periphery with $3 \mathrm{~d}$ structured illumination microscopy. Science, 320(5881):1332-1336, 2008. 43,65

[84] A. Stemmer, M. Beck, and R. Fiolka. Widefield fluorescence microscopy with extended resolution. Histochem. Cell Biol., 130:807-817, 2008. 43

[85] S. Habuchi, P. Dedecker, J. Hotta, C. Flors, R. Ando, H. Mizuno, A. Miyawaki, and J. Hofkens. Photo-induced protonation/deprotonation in the gfp-like fluorescent protein dronpa: mechanism responsible for the reversible photoswitching. Photochem. Photobiol. Sciences, 5:567-576, 2006. 47, 53

[86] M. Andresen, M. C. Wahl, A. C. Stiel, F. Gräter, L. V. Schäfer, S. Trowitzsch, G. Weber, C. Eggeling, H. Grubmüller, S. W. Hell, and S. Jakobs. Structure and mechanism of the reversible photoswitch of a fluorescent protein. Proc. Nat. Acad. Sci. U.S.A., 102(37):13070-13074, 2005. 53 
[87] M. Andresen, A. C. Stiel, S. Trowitzsch, G. Weber, C. Eggeling, M. C. Wahl, S. W. Hell, and S. Jakobs. Structural basis for reversible photoswitching in dronpa. Proc. Nat. Acad. Sci. U.S.A., 104:13005-13009, 2007. 53

[88] N. Bobroff. Position measurement with a resolution and noise-limited instrument. Rev. Sci. Instrum., 57(6):1152-1157, 1986. 55

[89] E. Betzig. Proposed method for molecular optical imaging. Opt. Lett., 20(3):237-239, 1995. 55

[90] S. W. Hell, J. Soukka, and P. E. Hänninen. Two- and multiphoton detection as an imaging mode and means of increasing the resolution in far-field light microscopy. Bioimaging, 3:65-69, 1995. 55

[91] J.A. Hogbom. Aperture synthesis with a non-regular distribution of interferometer baselines. Astron. Astrophys. Suppl., 15:417-426, 1974. 57

[92] B. Alberts, A. Johnson, J. Lewis, M. Raff, K. Roberts, and P. Walter. Molecular Biology of the Cell. Garland Science, New York, 4 edition, 2002. 63

[93] P. Dedecker, J. I. Hotta, C. Flors, M. Sliwa, H. Uji-I, M. B. J. Roeffaers, R. Ando, H. Mizuno, A. Miyawaki, and J. Hofkens. Subdiffraction imaging through the selective donut-mode depletion of thermally stable photoswitchable fluorophores: Numerical analysis and application to the fluorescent protein dronpa. J. Am. Chem. Soc., 129(51):16132-16141, 2007. 64

[94] C. Eggeling, A. Volkmer, and C. A. M. Seidel. Molecular photobleaching kinetics of rhodamine $6 \mathrm{~g}$ by one- and two-photon induced confocal fluorescence microscopy. ChemPhysChem, 6:791-804, 2005. 64

[95] R. A. Hoebe, C. H. Van Oven, T. W. J. Gadella Jr, P. B. Dhonukshe, C. J. F. Van Noorden, and E. M. M. Manders. Controlled light exposure microscopy (clem). Imaging and Microscopy, 9:32-24, 2007. 64

[96] D.M. Chudakov, V.V. Belousov, A.G. Zaraisky, V.V. Novoselov, D.B. Staroverov, D.B. Zorov, S. Lukyanov, and K.A. Lukyanov. Kindling fluorescent proteins for precise in vivo photolabeling. Nat. Biotechnol., 21:191-194, 2003. 65

[97] M. Andresen, A. C. Stiel, J. Fölling, D. Wenzel, A. Schönle, A. Egner, C. Eggeling, S. W. Hell, and S. Jakobs. Photoswitchable fluorescent proteins enable monochromatic multilable imaging and dual color fluorescence nanoscopy. Nat. Biotechnol., 26(9):1035 - 1040, 2008. 65, 81

[98] A. Punge, S. O. Rizzoli, A. Schönle, L. Kastrup, L. Meyer, J. D. Wildanger, R. Jahn, and S. W. Hell. 3d reconstruction of high-resolution sted microscope images. Micr. Res. Tech., 71:644-650, 2008. 65 
[99] E.A. Jares-Erijman and T. M. Jovin. Fret imaging. Nat. Biotechnol., 21(11):13871395, 2003. 68

[100] G. Donnert, J. Keller, C. A. Wurm, S. O. Rizzoli, V. Westphal, A. Schönle, R. Jahn, S. Jakobs, C. Eggeling, and S. W. Hell. Two-color far-field fluorescence nanoscopy. Biophys. J., 92(8):L67-69L, 2007. 68

[101] L. Meyer, D. Wildanger, R. Medda, A. Punge, S. O. Rizzoli, G. Donnert, and S. W. Hell. Dual-color sted microscopy at $30 \mathrm{~nm}$ focal plane resolution. Small, 2008. 68

[102] L.A. Ernst, R.K. Gupta, R.B. Mujumdar, and A.S. Waggoner. Cyanine dye labeling reagents for sulfhydryl-groups. Cytometry, 10:3-10, 1989. 68

[103] D.N. Dempster, T. Morrow, R. Rankin, and G.F. Thompson. Photochemical charcteristics of cyanine dyes .1. 3,3'-dyethyloxadicarbocyanine iodide. Journal of the Chemical Society - Faraday Transactions II, 68:1479, 1972. 70

[104] R. Zondervan, F. Kulzer, M.A. Kolchenko, and M. Orrit. Photobleaching of rhodamine $6 \mathrm{~g}$ in poly(vinyl alcohol) at the ensemble and single-molecule levels. J. Phys. Chem. A, 108:1657-1665, 2004. 70, 71

[105] Z. Huang, D. Ji, and A. Xia. Fluorescence intensity and lifetime fluctuations of single cy5 molecules immobilized on the glass surface. Coll. Surf. A, 257-258:203-209, 2005. 70

[106] V.I. Martynov, A.P. Savitsky, N.Y. Martynova, P.A. Savitsky, K.A. Lukyanov, and S.A. Lukyanov. Alternative cyclization in gfp-like proteins family. the formation and structure of the chromophore of a purple chromoprotein from anemonia sulcata. $J$. Biol. Chem., 276:21012-21016, 2001.75 


\section{A Appendix}

\section{A.1 Expressions for the Resolving Power}

In the following, an expression for the resolving power $\Delta x$ of the parallelized wide-field RESOLFT microscope shown in figure 2.12 is derived. The parameters are the switch-off intensity $\xi:=I_{\text {off }} / I_{\text {sat }}$, measured in multiples of the saturation intensity $I_{\text {sat }}$ (see (1.6), and the switch-off minimum $a:=I_{o f f}^{\min } / I_{\text {off }}^{\max }$, measured in multiples of the peak switch-off intensity. Two cases are considered: 1) a homogeneous probe beam and 2) a modulated probe beam (see 2.15). The expression derived here was used to generate the fits represented by the solid and the dashed curve in figure 2.14

In terms of the switch-off intensity distribution $I_{\text {off }}(x)$, the probe pattern $I_{\text {probe }}(x)$, and the depletion curve $\eta\left(I_{\text {off }}\right)$ of rsFastLime the fluorescence emission profile $F(x)$ measured in figure 2.13 can be written as

$$
F(x)=I_{\text {probe }}(x) \cdot \eta\left(I_{\text {off }}(x)\right) .
$$

Allowing for a non-zero intensity minimum $a$ the standing wave patterns $I_{\text {off }}(x)$ and $I_{\text {probe }}(x)$ can be modelled as

$$
\begin{aligned}
I_{\text {off }}(x) & =a+(1-a) \sin ^{2}(\pi x / D) & & \\
I_{\text {probe }}(x) & =a+(1-a) \cos ^{2}(\pi x / D) & & \text { (structured probing) } \\
I_{\text {probe }}(x) & =1 & & \text { (homogeneous probing). }
\end{aligned}
$$

Together with the expression 2.3 for the rsFastLime depletion curve $\eta(\xi)=r+(1-r) /(1+\xi)$ it is straightforward to calculate the full width at half maximum (FWHM) $\Delta x$ of the fluorescence emission profile $F(x)$ (see $(\mathrm{A} .1)$ ). To avoid confusion: $\Delta x$ shall be defined as the width of $F(x)$ at the value $m:=\left(F_{\min }+F_{\max }\right) / 2$ (as opposed to $m:=F_{\max } / 2$ ). For matters of completeness $\Delta x$ is calculated for the case of structured probing (see A.3) and homogeneous probing (see A.4).

Homogeneous probing:

$$
\Delta x / D=\frac{2}{\pi} \arcsin \left(\sqrt{\frac{1+a \xi}{2+(a+1) \xi}}\right) .
$$

Structured probing, $r=0$ :

$$
\Delta x / D=\frac{2}{\pi} \arcsin \left(\sqrt{\frac{(1-m(1+a \xi))}{(1-a)(1+m \xi)}}\right),
$$


where $m$ is given by

$$
m=\frac{1+a+\left(1+a^{2}\right) \xi}{2(1+a \xi)(1+\xi)} .
$$

Structured probing, $r>0$ :

$$
\Delta x / D=\frac{1}{\pi} \arccos \left(1+p-\sqrt{p^{2}-4 q}\right),
$$

where $m, p$ and $q$ are given by

$$
\begin{aligned}
m & =\frac{(1+a r \xi)(1+\xi)+a(1+r \xi)(1+a \xi)}{2(1+a \xi)(1+\xi)} \\
p & =\frac{1+\xi(m+r(a-1))}{(1-a) r \xi} \\
q & =\frac{m-1+(a(m-r) \xi)}{r \xi\left((1-a)^{2}\right)} .
\end{aligned}
$$

\section{A.2 Photophysical Model of rsFastLime}

In the following the fitting parameters obtained for the fits shown in figure 2.21 and figure 2.22 are listed. For all fits a fluorescence lifetime of $\tau_{f l}=2.7 \mathrm{~ns}$ and a fluorescence absorption cross section of $\sigma_{0} 1=1.4 \cdot 10^{-16} \mathrm{~cm}^{2}$ was assumed. These values had previously been obtained through TCSPC and absorption measurements with rsFastLime [69].

\begin{tabular}{|c|c|c|c|c|c|}
\hline figure & graph & $\Delta t_{\text {off }}[s]$ & $I_{\text {sat }}\left[W / \mathrm{cm}^{2}\right]$ & $k_{S D 2}[1 / s]$ & $k_{D 2 S}[1 / s]$ \\
\hline 2.21 & $\mathbf{a}$ & 0.01 & 56 & $2.0 \mathrm{e}+6$ & $8.0 \mathrm{e}+1$ \\
\hline & b & 0.1 & 7.9 & $1.2 \mathrm{e}+7$ & $1.5 \mathrm{e}+2$ \\
\hline & $\mathbf{c}$ & 1 & 0.9 & $5.0+6$ & $4.3 \mathrm{e}+1$ \\
\hline
\end{tabular}

\begin{tabular}{|c|c|c|c|c|c|c|}
\hline figure & graph & $\Delta t_{\text {off }}[\mathrm{s}]$ & $I_{\text {sat }}\left[\mathrm{W} / \mathrm{cm}^{2}\right]$ & $k_{S D 1}[1 / \mathrm{s}]$ & $\sigma_{D 1 S}\left[\mathrm{~cm}^{2}\right]$ & $\sigma_{D 1 B}\left[\mathrm{~cm}^{2}\right]$ \\
\hline 2.21 & a & 0.01 & 56 & $1.5 \mathrm{e}+6$ & $4.5 \mathrm{e}-20$ & $8.5 \mathrm{e}-22$ \\
\hline & b & 0.1 & 7.9 & $1.3 \mathrm{e}+6$ & $3.5 \mathrm{e}-21$ & $3.0 \mathrm{e}-22$ \\
\hline & $\mathbf{c}$ & 1 & 0.9 & $1.0 \mathrm{e}+6$ & $8.5-21$ & $1.0 \mathrm{e}-22$ \\
\hline
\end{tabular}

\begin{tabular}{|c|c|c|c|c|c|}
\hline figure & graph & $I_{o f f}\left[\mathrm{~kW} / \mathrm{cm}^{2}\right]$ & $t_{1 / 2}[\mathrm{~ms}]$ & $k_{S D 2}[1 / \mathrm{s}]$ & $k_{D 2 S}[1 / \mathrm{s}]$ \\
\hline 2.22 & $\mathbf{a}$ & 0.1 & 10 & $1.3 \mathrm{e}+6$ & $1.1 \mathrm{e}+1$ \\
\hline & $\mathbf{b}$ & 1 & 1.6 & $1.1 \mathrm{e}+6$ & $1.3 \mathrm{e}+2$ \\
\hline & $\mathbf{c}$ & 10 & 0.25 & $5.0+5$ & $1.8 \mathrm{e}+2$ \\
\hline
\end{tabular}

\begin{tabular}{|c|c|c|c|c|c|c|}
\hline figure & graph & $I_{\text {off }}\left[\mathrm{kW} / \mathrm{cm}^{2}\right]$ & $t_{1 / 2}[\mathrm{~ms}]$ & $k_{S D 1}[1 / \mathrm{s}]$ & $\sigma_{D 1 S}\left[\mathrm{~cm}^{2}\right]$ & $\sigma_{D 1 B}\left[\mathrm{~cm}^{2}\right]$ \\
\hline 2.22 & $\mathbf{a}$ & 0.1 & 10 & $7.5 \mathrm{e}+5$ & $9.0 \mathrm{e}-21$ & $1.7 \mathrm{e}-22$ \\
\hline & $\mathbf{b}$ & 1 & 1.6 & $3.8 \mathrm{e}+5$ & $5.0 \mathrm{e}-21$ & $1.5 \mathrm{e}-22$ \\
\hline & $\mathbf{c}$ & 10 & 0.25 & $1.5 \mathrm{e}+5$ & $3.5-21$ & $2.5 \mathrm{e}-22$ \\
\hline
\end{tabular}




\section{A.3 Staining of Tubulin in PtK2 Cells}

PtK2 (Potorous tridactylus) cells were grown on coverslips and fixed with $8 \%(\mathrm{w} / \mathrm{v})$ formaldehyde for three minutes. After postfixation with pure acetone for $5 \mathrm{~s}$ at $-20^{\circ} \mathrm{C}$ the cells were extracted for 10 minutes in 5\% (v/v) Triton X-100 in PBS (137mM NaCl, 3mM KCl, 8mM $\left.\mathrm{Na}_{2} \mathrm{HPO}_{4}, 1.5 \mathrm{mM} \mathrm{KH} \mathrm{KH}_{4}, \mathrm{pH} 7\right)$. Mictrotubules were decorated by incubation with an anti-beta-tubulin monoclonal antibody (Chemicon, Temecula, CA, USA). This antibody was detected by a Cy5-coupled anti-mouse antibody together with a biotinylated anti-mouse antibody (both Jackson ImmunoResearch Laboratories, Inc., West Grove, PA, USA). Subsequently, NeutrAvidin (Molecular Probes, Eugene, OR, USA) and biotinylated rsFastLime were added to the cells. For mounting of the cells a solution of $1 \%$ (w/v) PVA in PBS was added. Finally, the samples were spin-coated for 20s at 5000rpm (SpinCoater KW-4A, Chemat Technology, Inc., Northridge, CA, USA). 


\section{A.4 List of Scientific Contributions}

Parts of this thesis have been published as follows:

1. J. Fölling, M. Bossi, H. Bock, R. Medda, C. A. Wurm, B. Hein, S. Jakobs, C. Eggeling, S. W. Hell. Fluorescence nanoscopy by ground-state depletion and single-molecule return. Nat. Meth., 5 (11), 943-945, 2008

2. A.C. Stiel, M. Andresen, H. Bock, M. Hilbert, J. Schilde, A. Schönle, C. Eggeling, A. Egner, S.W. Hell, S. Jakobs. Generation of monomeric reversibly switchable red fluorescent proteins for far-field fluorescence nanoscopy. Biophys. J., 95 (6), 29892997, 2008

3. C. Eggeling, M. Hilbert, H. Bock, C. Ringemann, M. Hofmann, A.C. Stiel, M. Andresen, S. Jakobs, A. Egner, A. Schönle, S.W. Hell. Reversible photoswitching enables single-molecule fluorescence fluctuation Spectroscopy at high molecular concentration. Micr. Res. Tech., 70 (12), 1003-1009, 2007

4. A. Egner, C. Geisler, C. von Middendorff, H. Bock, D. Wenzel, R. Medda, M. Andresen, A.C. Stiel, S. Jakobs, C. Eggeling, A. Schönle, S.W. Hell. Fluorescence nanoscopy in whole cells by asynchronous localization of photoswitching emitters. Biophys. J., 93 (9), 3285-3290, 2007

5. C. Geisler, A. Schönle, C. von Middendorff, H. Bock, C. Eggeling, A. Egner, S.W. Hell. Resolution of lambda/10 in fluorescence microscopy using fast single molecule photo-switching. Appl. Phys. A, 88 (2), 223-226, 2007

6. H. Bock, C. Geisler, C.A. Wurm, C. von Middendorff, S. Jakobs, A. Schönle, A. Egner, S.W. Hell, C. Eggeling. Two-color far-field fluorescence nanoscopy based on photoswitchable emitters. Appl. Phys. B, 88 (2), 161-165, 2007

7. M.A. Schwentker, H. Bock, M. Hofmann, S. Jakobs, J. Bewersdorf, C. Eggeling, S.W. Hell. Wide-field subdiffraction RESOLFT microscopy using fluorescent protein photoswitching. Micr. Res. Tech., 70 (3), 269-280, 2007 


\section{B Danksagung}

Zuletzt möchte ich all denen danken, die zum Erfolg dieser Arbeit beigetragen haben.

Mein besonderer Dank gilt Prof. Dr. Stefan W. Hell, der mir das Thema dieser Dissertation vorgeschlagen hat. Seine Begeisterung und seine fachlichen Anregungen waren zugleich Motivation und eine große Hilfe bei der Arbeit. Weiterhin möchte ich ihm für die Bereitstellung dieses hervorragenden Arbeitsumfeldes danken. Außerdem herzlichen Dank für ca. 500 Liter Kaffee!

Meinem Betreuer Christian Eggeling, für ständige Unterstützung und Ermutigung, fachkundige Ratschläge, tolle Ideen und unendliche Geduld!

Prof. Dr. Markus Münzenberg dafür, daß er sich freundlicherweise als Referent für diese Arbeit zur Verfügung gestellt hat.

Stefan Jakobs, Martin Andresen, Andre Stiel, Tanja Brakemann und Tim Grotjohann für die zuverlässige Versorgung mit schaltbaren FPs.

Alexander Egner für die Programmierung der PALMIRA-Auswertesoftware, häufige Hilfestellung bei der Datenanalyse und für die geduldige Beantwortung zahlreicher Fachfragen.

Andreas Schönle für die Bereitstellung und ständige Verbesserung der Laborsoftware und hilfreiche Diskussionen.

Jan Keller und Claas von Middendorff für eine große Zahl von Programmierarbeiten und die Beantwortung vieler Fragen.

Jaydev Jethwa, Harald Meyer, Donald Ouw und Rainer Pick für kompetente, zuverlässige und immer freundliche Hilfe rund um Laser, Kühlungen, Klimaanlagen, Meßtische, Elektronik, Chemikalien, optische Komponenten und vieles mehr.

Christian Wurm und Rebecca Medda für die Versorgung mit Zellproben.

Claudia Geisler und Jonas Fölling für Hilfe rund um das PALMIRA-Projekt.

Silvia Löbermann und Rita Schmitz-Salue für Hilfe rund ums Bio-Labor. 
Den Damen und Herren der Feinmechanik-Werkstatt für die Ausstattung mit hochwertigen Bauteilen.

Meinen Labor- und Bureaukollegen im Laufe der Zeit: Miriam Schwentker, Michael Hofmann, Michael Hilbert, Stefan Bretschneider, Arnold Giske, Robert Kellner, Steffen Sahl, Marcel Leutenegger, Daniel Aquino, Dominik Wildanger und Christian Ringemann für ständige Hilfe und die freundschaftliche Atmosphäre.

Den Sekretärinnen Anja Dietrich, Sarah Aschemann und Josephine Stadler für ihre kompetente und schnelle Hilfe mit jedwedem Papierkram.

Gerhard König für die zahlreichen Pakete.

Allen übrigen Mitgliedern der Abteilung Nanobiophotonik für die schöne Zeit in den vergangenen Jahren.

Meinen Eltern, Dietke, Marcus und Janne für alles! 